\title{
Enantioselective Catalytic Cyclopropanation-Rearrangement Approach to Chiral Spiroketals
}

Kuiyong Dong, ${ }^{1}$ Raj Gurung, ${ }^{1}$ Xingfang Xu, ${ }^{2, *}$ and Micheal P. Doyle ${ }^{1, * *}$

${ }^{1}$ Department of Chemistry, University of Texas at San Antonio, San Antonio, Texas 78249, United States

${ }^{2}$ Guangdong Key Laboratory of Chiral Molecule and Drug Discovery, School of Pharmaceutical Sciences, Sun Yat-sen University, Guangzhou, 510006, China

Email: michael.doyle@utsa.edu, xuxinfang@mail.sysu.edu.cn 


\section{Table of Contents}

1. General Information S2

2. General Procedure for the Synthesis of Dihydrooxazole S3-S5

3. General Procedure for the Preparation of spiroketals $4 \quad$ S5-S16

4. NMR Spectra of New compounds S17-S41

5. HPLC Traces for Racemic and Chiral Compounds S42-S51

6. X-ray Refinement Data S52-S53

7. Reference S53-S54 


\section{General Information}

Unless otherwise noted, all reactions were performed in $10 \mathrm{~mL}$ oven-dried $\left(120{ }^{\circ} \mathrm{C}\right)$ glassware under a $\mathrm{N}_{2}$ atmosphere. Solvents were dried using a JC Meyer solvent purification system. Analytical thin-layer chromatography was performed using glass plates pre-coated with 200-300 mesh silica gel impregnated with a fluorescent indicator $(254 \mathrm{~nm})$. Column chromatography was performed on CombiFlash ${ }^{\circledR}$ Rf200 and $\mathrm{Rf}+$ purification systems using normal phase silica gel columns. (300-400 mesh). High-resolution mass spectra (HRMS) were obtained on a Bruker MicroTOF-ESI mass spectrometer with an ESI resource using CsI or LTQ ESI Positive Ion Calibration Solution as the standard. Accurate masses were reported for the molecular ions $[\mathrm{M}+\mathrm{H}]^{+}$ or $[\mathrm{M}+\mathrm{Na}]^{+}$. Melting points were obtained uncorrected from an Electro Thermo MelTemp DLX 104 device. ${ }^{1} \mathrm{H}$ NMR spectra were recorded on a Bruker spectrometer (500 $\mathrm{MHz}$ ). Chemical shifts were reported in ppm downfield from tetramethylsilane (TMS) with the solvent resonance as the internal standard $\left(\mathrm{CDCl}_{3}, \delta=7.26\right)$. Spectra were reported as follows: chemical shift $(\delta$ ppm$)$, multiplicity $(\mathrm{s}=$ singlet, $\mathrm{d}=$ doublet, $\mathrm{t}=$ triplet, $\mathrm{q}=$ quartet, $\mathrm{m}=$ multiplet, comp = composite of magnetically non-equivalent protons, $\mathrm{dd}=$ doublet of doublets), coupling constants $(\mathrm{Hz})$, integration and assignment. ${ }^{13} \mathrm{C}$ NMR spectra were collected on Bruker instruments $(125 \mathrm{MHz})$ with complete proton decoupling. Chemical shifts are reported in ppm from the tetramethylsilane with the solvent resonance as internal standard $(\mathrm{CDCl} 3, \delta=77.16)$. Enantioselectivities were determined by HPLC analysis at $25^{\circ} \mathrm{C}$ using an Agilent 1260 Infinity HPLC System equipped with an G1311B quaternary pump, G1315D diode array detector, G1329B auto-sampler, G1316A thermostated column compartment and G1170A valve drive. For instrument control and data processing, Agilent OpenLAB CDS ChemStation Edition for LC \& LC/MS Systems (Rev. C.01.07 [26]) software was used. Chiralpak OD-H or (R,R-Whelk-O1) columns.

Enoldiazo compounds 2 and diazo compound 5 were prepared according to the literature procedures, ${ }^{[1]}$ and their identities corresponded to those reported in the published literatures. 


\section{General procedure for the synthesis of substrates and}

characterization data for new substrates ${ }^{[2]}$<smiles>[R]C(=O)NC(C)(C)C#C</smiles><smiles>CCOCCO</smiles><smiles>[R]C1=NC(C)(C)C(=C)O1</smiles>

To a solution of the appropriate propargyl amide ( $3 \mathrm{mmol}, 1$ equiv) in $\mathrm{CH}_{2} \mathrm{Cl}_{2}(30 \mathrm{~mL})$, $\mathrm{ZnI}_{2}$ (1 equiv) solid was slowly added, and the reaction mixture was stirred at room temperature overnight. The resulting reaction mixture was diluted with water $(15 \mathrm{~mL})$. The aqueous layer was extracted with $\mathrm{CH}_{2} \mathrm{Cl}_{2}(3 \times 20 \mathrm{~mL})$, and the combined organic layers were washed with water and brine, dried over anhydrous $\mathrm{Na}_{2} \mathrm{SO}_{4}$, and concentrated under reduced pressure. Silica gel chromatography (ethyl acetate/hexane) gave the desired oxazoline derivatives.<smiles>C=C1OC(c2ccc(OC)cc2)=NC1(C)C</smiles>

\section{2-(4-Methoxyphenyl)-4,4-dimethyl-5-methylene-4,5-dihydrooxazole $1 \mathrm{~b}$}

Column chromatoghraphy (silica gel; hexane/ethyl acetate, 20:1); Colorless oil, $528 \mathrm{mg}$, $81 \%$ yield, ${ }^{1} \mathrm{H}$ NMR $\left(500 \mathrm{MHz}, \mathrm{CDCl}_{3}\right)(\delta, \mathrm{ppm}) 7.92(\mathrm{~d}, J=8.9 \mathrm{~Hz}, 2 \mathrm{H}), 6.92(\mathrm{~d}, J=$ $8.9 \mathrm{~Hz}, 2 \mathrm{H}), 4.70(\mathrm{~d}, J=2.8 \mathrm{~Hz}, 1 \mathrm{H}), 4.21(\mathrm{~d}, J=2.8 \mathrm{~Hz}, 1 \mathrm{H}), 3.83(\mathrm{~s}, 3 \mathrm{H}), 1.43$ (s, $6 \mathrm{H}) ;{ }^{13} \mathrm{C} \mathrm{NMR}\left(125 \mathrm{MHz}, \mathrm{CDCl}_{3}\right)(\delta, \mathrm{ppm}) 168.2,162.4,159.7,130.0,119.5,113.9$, 82.1, 69.0, 55.5, 29.9; HRMS (ESI) $m / z$ : $[\mathrm{M}+\mathrm{H}]^{+}$Calcd. for $\mathrm{C}_{13} \mathrm{H}_{16} \mathrm{NO}_{2} 218.1176$; Found: 218.1170.<smiles>C=C1OC(c2ccc(C(F)(F)F)cc2)=NC1(C)C</smiles>

\section{4,4-Dimethyl-5-methylene-2-[4-(trifluoromethyl)phenyl]-4,5-dihydrooxazole 1c} Column chromatoghraphy (silica gel; hexane/ethyl acetate, 20:1); Colorless oil, $673 \mathrm{mg}$, $88 \%$ yield, ${ }^{1} \mathrm{H}$ NMR $\left(500 \mathrm{MHz}, \mathrm{CDCl}_{3}\right)(\delta, \mathrm{ppm}) 8.10(\mathrm{~d}, J=8.3 \mathrm{~Hz}, 2 \mathrm{H}), 7.69(\mathrm{~d}, J=$ $8.3 \mathrm{~Hz}, 2 \mathrm{H}), 4.76(\mathrm{~d}, J=3.0 \mathrm{~Hz}, 1 \mathrm{H}), 4.28(\mathrm{~d}, J=3.0 \mathrm{~Hz}, 1 \mathrm{H}), 1.45(\mathrm{~s}, 6 \mathrm{H}) ;{ }^{13} \mathrm{C} \mathrm{NMR}$ $\left(125 \mathrm{MHz}, \mathrm{CDCl}_{3}\right)(\delta, \mathrm{ppm}) 167.7,158.9,133.4(\mathrm{q}, J=32.7 \mathrm{~Hz}), 130.5,128.6,125.6$ (q, $J=3.4 \mathrm{~Hz}$ ), 123.9 (q, $J=272.4 \mathrm{~Hz}$ ), 83.1, 69.5, 29.8; HRMS (ESI) $\mathrm{m} / z:[\mathrm{M}+\mathrm{H}]^{+}$ Calcd. for $\mathrm{C}_{13} \mathrm{H}_{13} \mathrm{~F}_{3} \mathrm{NO} 256.0944$; Found: 256.0942 .<smiles>C=C1OC(c2ccc(Br)cc2)=NC1(C)C</smiles>

\section{2-(4-Bromophenyl)-4,4-dimethyl-5-methylene-4,5-dihydrooxazole 1d}

Column chromatoghraphy (silica gel; hexane/ethyl acetate, 20:1); Colorless oil, $644 \mathrm{mg}$, 
$81 \%$ yield, ${ }^{1} \mathrm{H}$ NMR $\left(500 \mathrm{MHz}, \mathrm{CDCl}_{3}\right)(\delta, \mathrm{ppm}) 7.84(\mathrm{~d}, J=8.3 \mathrm{~Hz}, 2 \mathrm{H}), 7.57(\mathrm{~d}, J=$ $8.3 \mathrm{~Hz}, 2 \mathrm{H}), 4.73(\mathrm{~d}, J=2.1 \mathrm{~Hz}, 1 \mathrm{H}), 4.25(\mathrm{~d}, J=2.1 \mathrm{~Hz}, 1 \mathrm{H}), 1.44(\mathrm{~s}, 6 \mathrm{H}) ;{ }^{13} \mathrm{C} \mathrm{NMR}$ $\left(125 \mathrm{MHz}, \mathrm{CDCl}_{3}\right)(\delta, \mathrm{ppm}) 167.9,159.2,131.9,129.7,126.5,126.1,82.8,69.4,29.8$; HRMS (ESI) $m / z$ : [M+H] $]^{+}$Calcd. for $\mathrm{C}_{12} \mathrm{H}_{13} \mathrm{BrNO} 266.0175$; Found: 266.0168 .<smiles>C=C1OC(c2ccccc2Br)=NC1(C)C</smiles>

\section{2-(2-Bromophenyl)-4,4-dimethyl-5-methylene-4,5-dihydrooxazole $1 \mathrm{e}$}

Column chromatoghraphy (silica gel; hexane/ethyl acetate, 20:1); Colorless oil, $798 \mathrm{mg}$, 89\% yield, ${ }^{1} \mathrm{H}$ NMR $\left(500 \mathrm{MHz}, \mathrm{CDCl}_{3}\right)(\delta, \mathrm{ppm}) 7.70(\mathrm{dd}, J=7.6,1.8 \mathrm{~Hz}, 1 \mathrm{H}), 7.65$ $(\mathrm{dd}, J=7.9,1.3 \mathrm{~Hz}, 1 \mathrm{H}), 7.36(\mathrm{td}, J=7.6,1.3 \mathrm{~Hz}, 1 \mathrm{H}), 7.30(\mathrm{td}, J=7.9,1.8 \mathrm{~Hz}, 1 \mathrm{H})$, $4.72(\mathrm{~d}, J=2.9 \mathrm{~Hz}, 1 \mathrm{H}), 4.26(\mathrm{~d}, J=2.9 \mathrm{~Hz}, 1 \mathrm{H}), 1.48(\mathrm{~s}, 6 \mathrm{H}) ;{ }^{13} \mathrm{C}$ NMR $(125 \mathrm{MHz}$, $\left.\mathrm{CDCl}_{3}\right)(\delta, \mathrm{ppm}) 167.8,159.3,134.0,132.0,131.4,127.6,127.3,122.1,82.8,69.7,29.7$; HRMS (ESI) $m / z$ : [M+H] ${ }^{+}$Calcd. for $\mathrm{C}_{12} \mathrm{H}_{13} \mathrm{BrNO} 266.0175$; Found: 266.0168.<smiles>C=C1OC(c2ccc(Br)cc2Cl)=NC1(C)C</smiles>

\section{2-(4-Bromo-2-chlorophenyl)-4,4-dimethyl-5-methylene-4,5-dihydrooxazole $1 \mathrm{f}$}

Column chromatoghraphy (silica gel; hexane/ethyl acetate, 20:1); Colorless oil, $780 \mathrm{mg}$, $87 \%$ yield, ${ }^{1} \mathrm{H}$ NMR $\left(500 \mathrm{MHz}, \mathrm{CDCl}_{3}\right.$ ) $(\delta, \mathrm{ppm}) 77.71-7.62$ (comp, 2H), $7.49-7.43$ $(\mathrm{m}, 1 \mathrm{H}), 4.72(\mathrm{~d}, J=3.0 \mathrm{~Hz}, 1 \mathrm{H}), 4.26(\mathrm{~d}, J=3.0 \mathrm{~Hz}, 1 \mathrm{H}), 1.46(\mathrm{~s}, 6 \mathrm{H}) ;{ }^{13} \mathrm{C}$ NMR $(125$ $\mathrm{MHz}, \mathrm{CDCl}_{3}$ ) $(\delta$, ppm) 167.5, 157.8, 134.7, 133.6, 132.4, 130.1, 125.8, 125.6, 83.0, 69.8, 29.7; HRMS (ESI) $m / z$ : $[\mathrm{M}+\mathrm{H}]^{+}$Calcd. for $\mathrm{C}_{12} \mathrm{H}_{12} \mathrm{BrClNO}$ 299.9785; Found: 299.9779.

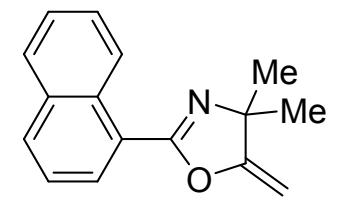

\section{4,4-Dimethyl-5-methylene-2-(naphthalen-1-yl)-4,5-dihydrooxazole $1 \mathrm{~g}$}

Column chromatoghraphy ( silica gel; hexane/ethyl acetate, 20:1); Colorless oil, $569 \mathrm{mg}$, $80 \%$ yield, ${ }^{1} \mathrm{H}$ NMR $\left(500 \mathrm{MHz}, \mathrm{CDCl}_{3}\right)(\delta, \mathrm{ppm}) 9.17(\mathrm{~d}, J=8.6 \mathrm{~Hz}, 1 \mathrm{H}), 8.21-8.13$ (m, 1H), $7.98(\mathrm{~d}, J=8.2 \mathrm{~Hz}, 1 \mathrm{H}), 7.89(\mathrm{~d}, J=8.1 \mathrm{~Hz}, 1 \mathrm{H}), 7.68-7.61(\mathrm{~m}, 1 \mathrm{H}), 7.59-$ 7.49 (comp, 2H), 4.80 (d, $J=2.8 \mathrm{~Hz}, 1 \mathrm{H}), 4.31$ (d, $J=2.8 \mathrm{~Hz}, 1 \mathrm{H}), 1.57$ (s, 6H); ${ }^{13} \mathrm{C}$ NMR $\left(125 \mathrm{MHz}, \mathrm{CDCl}_{3}\right)(\delta, \mathrm{ppm}) 167.4,159.6,133.9,132.3,131.2,129.2,128.6$, 127.7, 126.4, 126.3, 124.7, 123.7, 82.1, 70.1, 30.0; HRMS (ESI) $m / z:[\mathrm{M}+\mathrm{H}]^{+}$Calcd. for $\mathrm{C}_{16} \mathrm{H}_{16} \mathrm{NO} 238.1226$; Found: 238.1222 . 
<smiles>C=C1OC(c2ccco2)=NC1(C)C</smiles>

\section{2-(Furan-2-yl)-4,4-dimethyl-5-methylene-4,5-dihydrooxazole $1 \mathrm{~h}$}

Column chromatoghraphy (silica gel; hexane/ethyl acetate, 20:1); Colorless oil, $452 \mathrm{mg}$, 85\% yield, ${ }^{1} \mathrm{H}$ NMR $\left(500 \mathrm{MHz}, \mathrm{CDCl}_{3}\right)(\delta, \mathrm{ppm}) 7.58-7.52(\mathrm{~m}, 1 \mathrm{H}), 7.00(\mathrm{~d}, J=3.4$ $\mathrm{Hz}, 1 \mathrm{H}), 6.60-6.41(\mathrm{~m}, 1 \mathrm{H}), 4.72(\mathrm{~d}, J=3.0 \mathrm{~Hz}, 1 \mathrm{H}), 4.24(\mathrm{~d}, J=3.0 \mathrm{~Hz}, 1 \mathrm{H}), 1.43$ $(\mathrm{s}, 6 \mathrm{H}) ;{ }^{13} \mathrm{C} \mathrm{NMR}\left(125 \mathrm{MHz}, \mathrm{CDCl}_{3}\right)(\delta, \mathrm{ppm}) 167.3,152.5,145.7,142.2,115.0,111.8$, 83.0, 69.1, 29.8; HRMS (ESI) $m / z$ : $[\mathrm{M}+\mathrm{H}]^{+}$Calcd. for $\mathrm{C}_{10} \mathrm{H}_{12} \mathrm{NO}_{2}$ 178.0863; Found: 178.0858 .<smiles>C=C1OC(/C=C/c2ccccc2)=NC1(C)C</smiles>

\section{(E)-4,4-Dimethyl-5-methylene-2-styryl-4,5-dihydrooxazole 1i}

Column chromatoghraphy (silica gel; hexane/ethyl acetate, 20:1); Colorless oil, $575 \mathrm{mg}$, 90\% yield, ${ }^{1} \mathrm{H}$ NMR $\left(500 \mathrm{MHz}, \mathrm{CDCl}_{3}\right)(\delta, \mathrm{ppm}) 7.52-7.48$ (comp, $\left.2 \mathrm{H}\right), 7.44$ (d, $J=$ $16.3 \mathrm{~Hz}, 1 \mathrm{H}), 7.40-7.32$ (comp, 3H), 6.61 (d, $J=16.3 \mathrm{~Hz}, 1 \mathrm{H}), 4.69$ (d, $J=2.9 \mathrm{~Hz}$, $1 \mathrm{H}), 4.21(\mathrm{~d}, J=2.9 \mathrm{~Hz}, 1 \mathrm{H}), 1.41(\mathrm{~s}, 6 \mathrm{H}) ;{ }^{13} \mathrm{C} \mathrm{NMR}\left(125 \mathrm{MHz}, \mathrm{CDCl}_{3}\right)(\delta, \mathrm{ppm})$ 167.7, 159.8, 140.8, 135.1, 129.8, 129.0, 127.6, 114.5, 82.1, 68.9, 29.8; HRMS (ESI) $m / z:[\mathrm{M}+\mathrm{H}]^{+}$Calcd. for $\mathrm{C}_{14} \mathrm{H}_{16} \mathrm{NO} 214.1226$; Found: 214.1221 .

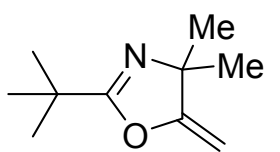

\section{2-(tert-Butyl)-4,4-dimethyl-5-methylene-4,5-dihydrooxazole $\mathbf{1 j}$}

Column chromatoghraphy (silica gel; hexane/ethyl acetate, 10:1); Colorless oil, $326 \mathrm{mg}$, $65 \%$ yield, ${ }^{1} \mathrm{H}$ NMR $\left(500 \mathrm{MHz}, \mathrm{CDCl}_{3}\right)(\delta, \mathrm{ppm}) 4.58(\mathrm{~d}, J=2.7 \mathrm{~Hz}, 1 \mathrm{H}), 4.13(\mathrm{~d}, J=$ $2.7 \mathrm{~Hz}, 1 \mathrm{H}), 1.35$ (s, 6H), 1.27 (s, 9H); ${ }^{13} \mathrm{C} \mathrm{NMR}\left(125 \mathrm{MHz}, \mathrm{CDCl}_{3}\right)(\delta, \mathrm{ppm})$ 174.1, 168.6, 81.2, 68.4, 29.9, 27.5, 26.6; HRMS (ESI) $m / z$ : $[\mathrm{M}+\mathrm{H}]^{+}$Calcd. for $\mathrm{C}_{10} \mathrm{H}_{18} \mathrm{NO}$ 168.1383; Found: 168.1378.

\section{General Procedure for the Preparation of Spiroketal 4.}

Table S1 Optimization of Spiroketal 4aa of 1a and 5a.

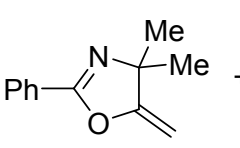

$1 \mathbf{a}$

\begin{tabular}{lllll}
\hline Entry $^{a}$ & $\mathrm{Rh}(\mathrm{II})$ & solvent & yield (\%) & ee $(\%)^{c}$ \\
\hline 1 & $\mathrm{Rh}_{2}(\mathrm{OAc})_{4}$ & $\mathrm{DCM}$ & 67 & - \\
2 & $\mathrm{Rh}_{2}(\mathrm{~S}-\mathrm{PTTL})_{4}$ & $\mathrm{DCM}$ & 85 & 60 \\
3 & $\mathrm{Rh}_{2}(\mathrm{~S}-\mathrm{TFPTTL})_{4}$ & $\mathrm{DCM}$ & 88 & 49
\end{tabular}




\begin{tabular}{|c|c|c|c|}
\hline 4 & $\mathrm{Rh}_{2}(\mathrm{~S}-\mathrm{TCPTTL})_{4}$ & DCM & 92 \\
\hline 5 & $\mathrm{Rh}_{2}(\mathrm{~S}-\mathrm{TBPTTL})_{4}$ & DCM & 91 \\
\hline 6 & $\mathrm{Rh}_{2}(\mathrm{~S}-\mathrm{NTTL})_{4}$ & DCM & 93 \\
\hline 7 & $\mathrm{Rh}_{2}(\mathrm{~S}-\mathrm{PTAD})_{4}$ & $\mathrm{DCM}$ & 82 \\
\hline 8 & $\mathrm{Rh}_{2}(\mathrm{~S}-\mathrm{DOSP})_{4}$ & $\mathrm{DCM}$ & NR \\
\hline 9 & $\mathrm{Rh}_{2}(\mathrm{~S}-\mathrm{BTPCP})_{4}$ & $\mathrm{DCM}$ & 88 \\
\hline 10 & $\mathrm{Rh}_{2}(\mathrm{~S}-\mathrm{NTTL})_{4}$ & DCE & 85 \\
\hline 11 & $\mathrm{Rh}_{2}(\mathrm{~S}-\mathrm{NTTL})_{4}$ & $\mathrm{CHCl}_{3}$ & 86 \\
\hline 12 & $\mathrm{Rh}_{2}(\mathrm{~S}-\mathrm{NTTL})_{4}$ & TBME & 80 \\
\hline 13 & $\mathrm{Rh}_{2}(\mathrm{~S}-\mathrm{NTTL})_{4}$ & Toluene & 90 \\
\hline 14 & $\mathrm{Rh}_{2}(\mathrm{~S}-\mathrm{NTTL})_{4}$ & Cyclohexane & 78 \\
\hline 15 & $\mathrm{Rh}_{2}(\mathrm{~S}-\mathrm{NTTL})_{4}$ & $\mathrm{Et}_{2} \mathrm{O}$ & 85 \\
\hline $16^{d}$ & $\mathrm{Rh}_{2}(\mathrm{~S}-\mathrm{NTTL})_{4}$ & DCM & 90 \\
\hline $17^{e}$ & $\mathrm{Rh}_{2}(\mathrm{~S}-\mathrm{NTTL})_{4}$ & DCM & 91 \\
\hline
\end{tabular}

[a] Reactions were carried out at room temperature on a $0.1 \mathrm{mmol}$ scale of 1a $(0.1 \mathrm{~mol})$ with dirhodium catalyst $(1.0 \mathrm{~mol} \%)$, in $1.0 \mathrm{~mL}$ solvent; 0.15 mmol of $5 \mathrm{a}$ in $1.0 \mathrm{~mL}$ same solvent was added over 60 min via a syringe pump. ${ }^{[b]}$ Yields were determined by ${ }^{1} \mathrm{H}$ NMR spectroscopic analysis of reaction mixture using 1,3,5-trimethoxybenzene as the internal standard. [c] The $e e$ 's were determined by chiral HPLC analysis, ODH column, 97:3 (hexanes: $\left.{ }^{i} \mathrm{PrOH}\right)$. [d] The reaction was carried out at $0{ }^{\circ} \mathrm{C}$; ${ }^{[\mathrm{e}]}$ The reaction was carried out at $40^{\circ} \mathrm{C}$.

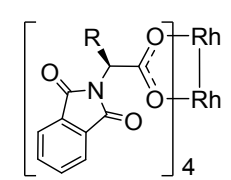

$\mathrm{R}=t \mathrm{Bu} \quad \mathrm{Rh}_{2}(\mathrm{~S}-\mathrm{PTTL})_{4}$ $\mathrm{R}=$ adamantyl $\mathrm{Rh}_{2}(\mathrm{~S}-\mathrm{PTAD})_{4}$

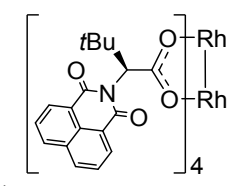

$\mathrm{Rh}_{2}(\mathrm{~S}-\mathrm{NTTL})_{4}$

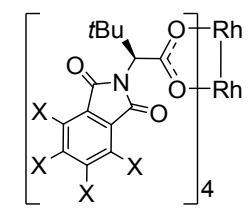

$\mathrm{X}=\mathrm{F}, \mathrm{Rh}_{2}(\mathrm{~S} \text {-TFPTTL })_{4}$ $\mathrm{X}=\mathrm{Cl}, \mathrm{Rh}_{2}(\mathrm{~S} \text {-TCPTTL })_{4}$ $\mathrm{X}=\mathrm{Br}, \mathrm{Rh}_{2}(S \text {-TBPTTL })_{4}$

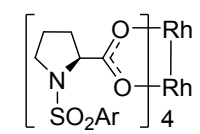

$\mathrm{Rh}_{2}(\mathrm{~S} \text {-DOSP })_{4}$ $\mathrm{Ar}=p-\left(n \mathrm{C}_{12} \mathrm{H}_{25}\right) \mathrm{C}_{6} \mathrm{H}_{4}$

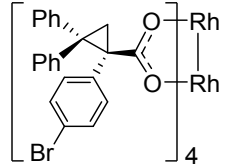

$\mathrm{Rh}_{2}(\mathrm{~S} \text {-BTPCP })_{4}$

\subsection{General Procedure for the Preparation of Spiroketal 4 from 1 and 2.}<smiles>C=C1OC([Al])=NC1(C)C</smiles>

1<smiles>[R16]OC(=[W])C(=C[PH2+])O[R]</smiles>

2
(1) $\mathrm{Rh}_{2}(\mathrm{~S} \text {-TCPTTL })_{4}(1.0 \mathrm{~mol} \%)$ DCM, rt, overnight

(2) TABF (2.0 eq), DCM, $5 \mathrm{~min}$<smiles>[R]OC(=O)C1=C([R])OC2(OC([Bi])=NC2[R])O1</smiles>

4

To a $10-\mathrm{mL}$ oven-dried vial containing a magnetic stirring bar were added $\mathrm{Rh}_{2}(S$ TCPTTL $)_{4}(3.6 \mathrm{mg}, 1.0 \mathrm{~mol} \%)$ and 5-methylene-4,5-dihydrooxazole $1(0.2 \mathrm{mmol})$ in dry DCM $(2.0 \mathrm{~mL})$. Then enoldiazo compound $2(0.3 \mathrm{mmol})$ in dry DCM $(2.0 \mathrm{~mL})$ was added as a solution via syringe pump over $1 \mathrm{~h}$ under an argon atmosphere at room temperature. After addition, the reaction solution was stirred overnight under these conditions until consumption of the reactants (monitored by TLC) was complete. Then the reaction vial was moved to an ice bath, and $0.4 \mathrm{~mL} \mathrm{TABF} \mathrm{(1.0} M$ in THF) solution was slowly addition to reaction mixture. The reaction solution was stirred at $0{ }^{\circ} \mathrm{C}$ until consumption of the reactants (monitored by TLC) was complete (about $5 \mathrm{~min}$ ). Then the reaction solution was purified by column chromatography on silica gel without any additional treatment (hexanes : EtOAc $=10: 1$ to $5: 1$ ) to give 4 as pure products. 
<smiles>CC(=O)C1=C(C)O[C@]2(C1)OC(c1ccccc1)=NC2(C)C</smiles>

\section{Methyl (S)-4,4,7-Trimethyl-2-phenyl-1,6-dioxa-3-azaspiro[4.4]nona-2,7-diene-8- carboxylate 4 aa}

Column chromatoghraphy (silica gel; hexane/ethyl acetate, 10:1); Colorless oil, 49.4 mg, 82\% yield, 99\% ee; [HPLC: Chiralpak OD-H column, 2\% IPA in hexane (v/v), 1.0 $\mathrm{mL} / \mathrm{min}, 250 \mathrm{~nm}, \mathrm{t}_{1}=7.9 \mathrm{~min}$ (major), $\mathrm{t}_{2}=5.6 \mathrm{~min}$ (minor) $] ;{ }^{1} \mathrm{H} \mathrm{NMR}\left(500 \mathrm{MHz}, \mathrm{CDCl}_{3}\right.$ ) ( $\delta$, ppm) $7.99-7.87$ (comp, 2H), $7.52-7.33$ (comp, 3H), 3.74 (s, 3H), $3.27-3.17$ (m, $1 \mathrm{H}), 3.16-3.06(\mathrm{~m}, 1 \mathrm{H}), 2.23(\mathrm{t}, J=1.8 \mathrm{~Hz}, 3 \mathrm{H}), 1.42(\mathrm{~s}, 3 \mathrm{H}), 1.27(\mathrm{~s}, 3 \mathrm{H}) ;{ }^{13} \mathrm{C}$ NMR $\left(125 \mathrm{MHz}, \mathrm{CDCl}_{3}\right)(\delta, \mathrm{ppm}) 165.8,165.7,160.4,131.7,128.4,128.3,127.5,118.6$, 101.8, 77.6, 51.2, 34.6, 25.7, 21.7, 14.0; HRMS (ESI) $\mathrm{m} / \mathrm{z}:[\mathrm{M}+\mathrm{H}]^{+}$Calcd. for $\mathrm{C}_{17} \mathrm{H}_{20} \mathrm{NO}_{4}: 302.1387$; Found 302.1382<smiles>CC1=C(C)C(C(=O)OCC(F)F)=C(C)[C@@]2(OC(c3ccccc3)=NC2(C)C)O1</smiles>

2,2,2-Trifluoroethyl $(S)-4,4,7-T r i m e t h y l-2-p h e n y l-1,6-d i o x a-3-a z a s p i r o[4.4]$ nona2,7-diene-8-carboxylate 4ab

Column chromatoghraphy (silica gel; hexane/ethyl acetate, 10:1); Colorless oil, 63.5 mg, 86\% yield, 99\% ee; [HPLC: Chiralpak OD-H column, 3\% IPA in hexane (v/v), 1.0 $\mathrm{mL} / \mathrm{min}, 250 \mathrm{~nm}, \mathrm{t}_{1}=6.0 \mathrm{~min}$ (major), $\mathrm{t}_{2}=4.6 \mathrm{~min}$ (minor) $] ;{ }^{1} \mathrm{H} \mathrm{NMR}\left(500 \mathrm{MHz}, \mathrm{CDCl}_{3}\right.$ )

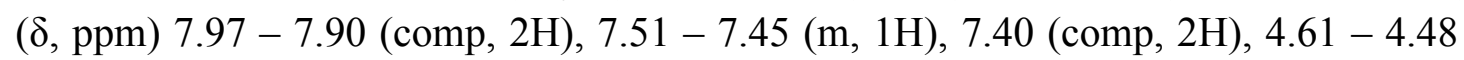
(comp, 2H), $3.29-3.22(\mathrm{~m}, 1 \mathrm{H}), 3.20-3.14(\mathrm{~m}, 1 \mathrm{H}), 2.26(\mathrm{t}, J=1.8 \mathrm{~Hz}, 3 \mathrm{H}), 1.44(\mathrm{~s}$, $3 \mathrm{H}), 1.30(\mathrm{~s}, 3 \mathrm{H}) ;{ }^{13} \mathrm{C} \mathrm{NMR}\left(125 \mathrm{MHz}, \mathrm{CDCl}_{3}\right)(\delta, \mathrm{ppm}) 168.3,163.2,160.4,131.8$, $128.5,128.3,127.4,123.4$ (q, $J=277.3 \mathrm{~Hz}), 119.2,100.6,70.9,59.9$ (q, $J=36.4 \mathrm{~Hz}$ ), 34.2, 25.7, 21.7, 14.4; HRMS (ESI) $m / z$ : $[\mathrm{M}+\mathrm{H}]^{+}$Calcd. for $\mathrm{C}_{18} \mathrm{H}_{19} \mathrm{~F}_{3} \mathrm{NO}_{4}$ 370.1261; Found 370.1251<smiles>CC1=C(C)[C@@]2(OC(c3ccccc3)=NC2(C)C)C(C)=C1C(=O)OCc1ccccc1</smiles>

Benzyl (S)-4,4,7-Trimethyl-2-phenyl-1,6-dioxa-3-azaspiro[4.4]nona-2,7-diene-8carboxylate 4ac

Column chromatoghraphy (silica gel; hexane/ethyl acetate, 10:1); Colorless solid, m.p: $87-88{ }^{\circ} \mathrm{C}, 59.6 \mathrm{mg}, 79 \%$ yield, 96\% ee; [HPLC: Chiralpak OD-H column, 3\% IPA in hexane (v/v), $2.0 \mathrm{~mL} / \mathrm{min}, 250 \mathrm{~nm}, \mathrm{t}_{1}=10.3 \mathrm{~min}$ (major), $\mathrm{t}_{2}=6.9 \mathrm{~min}($ minor $\left.)\right] ;{ }^{1} \mathrm{H} \mathrm{NMR}$ $\left(500 \mathrm{MHz}, \mathrm{CDCl}_{3}\right)(\delta, \mathrm{ppm}) 7.97-7.92$ (comp, $\left.2 \mathrm{H}\right), 7.50-7.45(\mathrm{~m}, 1 \mathrm{H}), 7.43-7.32$ (comp, 7H), $5.24(\mathrm{~d}, J=12.4 \mathrm{~Hz}, 1 \mathrm{H}), 5.21(\mathrm{~d}, J=12.4 \mathrm{~Hz}, 1 \mathrm{H}), 3.29-3.22(\mathrm{~m}, 1 \mathrm{H})$, $3.21-3.13(\mathrm{~m}, 1 \mathrm{H}), 2.26(\mathrm{t}, J=1.8 \mathrm{~Hz}, 3 \mathrm{H}), 1.44(\mathrm{~s}, 3 \mathrm{H}), 1.29(\mathrm{~s}, 3 \mathrm{H}) ;{ }^{13} \mathrm{C} \mathrm{NMR}(125$ 
$\left.\mathrm{MHz}, \mathrm{CDCl}_{3}\right)(\delta, \mathrm{ppm}) 166.2,165.1,160.5,136.5,131.7,128.7,128.5,128.3,128.3$, $128.2,127.5,118.9,101.8,70.7,65.8,34.6,25.7,21.8,14.2 ;$ HRMS (ESI) $m / z:[\mathrm{M}+\mathrm{H}]^{+}$ Calcd. for $\mathrm{C}_{23} \mathrm{H}_{24} \mathrm{NO}_{4} 378.1700$; Found 378.1691.<smiles>CC1=C(C(=O)OCc2ccc(C)cc2)O[C@@]2(C)OC(c3ccccc3)=NC12C</smiles>

Benzyl (S)-4,4,7-Trimethyl-2-phenyl-1,6-dioxa-3-azaspiro[4.4]nona-2,7-diene-8carboxylate 4ad

Column chromatoghraphy (silica gel; hexane/ethyl acetate, 10:1); Colorless oil, 63.4 mg, 81\% yield, 98\% ee; [HPLC: Chiralpak OD-H column, 3\% IPA in hexane (v/v), 1.0 $\mathrm{mL} / \mathrm{min}, 250 \mathrm{~nm}, \mathrm{t}_{1}=8.0 \mathrm{~min}$ (major), $\mathrm{t}_{2}=6.5 \mathrm{~min}$ (minor) $] ;{ }^{1} \mathrm{H} \mathrm{NMR}\left(500 \mathrm{MHz}, \mathrm{CDCl}_{3}\right.$ )

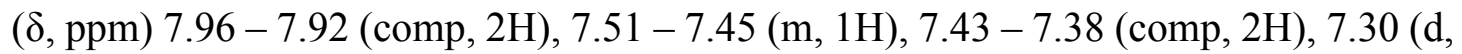
$J=8.0 \mathrm{~Hz}, 2 \mathrm{H}), 7.19(\mathrm{~d}, J=8.0 \mathrm{~Hz}, 2 \mathrm{H}), 5.20(\mathrm{~d}, J=12.2 \mathrm{~Hz}, 1 \mathrm{H}), 5.16(\mathrm{~d}, J=12.2$ $\mathrm{Hz}, 1 \mathrm{H}), 3.29-3.21(\mathrm{~m}, 1 \mathrm{H}), 3.19-3.12(\mathrm{~m}, 1 \mathrm{H}), 2.37(\mathrm{~s}, 3 \mathrm{H}), 2.25(\mathrm{t}, J=1.8 \mathrm{~Hz}$, $3 \mathrm{H}), 1.43(\mathrm{~s}, 3 \mathrm{H}), 1.28(\mathrm{~s}, 3 \mathrm{H}) ;{ }^{13} \mathrm{C} \mathrm{NMR}\left(125 \mathrm{MHz}, \mathrm{CDCl}_{3}\right)(\delta, \mathrm{ppm}) 166.1,165.2$, $160.5,138.1,133.5,131.7,129.4,128.5,128.5,128.3,127.5,118.9,101.9,70.7,65.8$, 34.7, 25.7, 21.8, 21.3, 14.2; HRMS (ESI) $\mathrm{m} / z$ : $[\mathrm{M}+\mathrm{H}]^{+}$Calcd. for $\mathrm{C}_{24} \mathrm{H}_{26} \mathrm{NO}_{4} 392.1856$; Found 392.1848.<smiles></smiles>

4-Methoxybenzyl (S)-4,4,7-Trimethyl-2-phenyl-1,6-dioxa-3-azaspiro[4.4]nona2,7-diene-8-carboxylate 4ae

Column chromatoghraphy (silica gel; hexane/ethyl acetate, 5:1); Colorless oil, $63.5 \mathrm{mg}$, 78\% yield, 98\% ee; [HPLC: Chiralpak OD-H column, 3\% IPA in hexane (v/v), 1.0 $\mathrm{mL} / \mathrm{min}, 250 \mathrm{~nm}, \mathrm{t}_{1}=14.2 \min$ (major), $\mathrm{t}_{2}=9.0 \min$ (minor)]; ${ }^{1} \mathrm{H}$ NMR $(500 \mathrm{MHz}$, $\left.\mathrm{CDCl}_{3}\right)(\delta, \mathrm{ppm}) 7.97-7.91$ (comp, 2H), $7.50-7.45(\mathrm{~m}, 1 \mathrm{H}), 7.43-7.38$ (comp, 2H), $7.35(\mathrm{~d}, J=8.7 \mathrm{~Hz}, 2 \mathrm{H}), 6.91(\mathrm{~d}, J=8.7 \mathrm{~Hz}, 2 \mathrm{H}), 5.17(\mathrm{~d}, J=12.1 \mathrm{~Hz}, 1 \mathrm{H}), 5.14(\mathrm{~d}, J$ $=12.1 \mathrm{~Hz}, 1 \mathrm{H}), 3.81(\mathrm{~s}, 3 \mathrm{H}), 3.27-3.19(\mathrm{~m}, 1 \mathrm{H}), 3.18-3.11(\mathrm{~m}, 1 \mathrm{H}), 2.24(\mathrm{t}, J=1.8$ $\mathrm{Hz}, 3 \mathrm{H}), 1.42$ (s, 3H), $1.28(\mathrm{~s}, 3 \mathrm{H}) ;{ }^{13} \mathrm{C} \mathrm{NMR}\left(125 \mathrm{MHz}, \mathrm{CDCl}_{3}\right)(\delta, \mathrm{ppm}) 166.0,165.2$, $160.4,159.7,131.7,130.2,128.6,128.4,128.3,127.5,118.9,114.0,101.9,70.7,65.6$, 55.4, 34.7, 25.7, 21.7, 14.2; HRMS (ESI) $m / z$ : $[\mathrm{M}+\mathrm{H}]^{+}$Calcd. for $\mathrm{C}_{24} \mathrm{H}_{26} \mathrm{NO}_{5} 408.1805$; Found 408.1797.<smiles>CC1=C(C)[C@@]2(OC(c3ccccc3)=NC2(C)C)C(C)=C1C(=O)OCc1ccc(Br)cc1</smiles>

4-Bromobenzyl (S)-4,4,7-Trimethyl-2-phenyl-1,6-dioxa-3-azaspiro[4.4]nona-2,7diene-8-carboxylate 4 af 
Column chromatoghraphy (silica gel; hexane/ethyl acetate, 10:1); Colorless oil, 72.8 mg, 80\% yield, 98\% ee; [HPLC: Chiralpak OD-H column, 3\% IPA in hexane (v/v), 1.0 $\mathrm{mL} / \mathrm{min}, 250 \mathrm{~nm}, \mathrm{t}_{1}=9.8 \mathrm{~min}$ (major), $\mathrm{t}_{2}=7.3 \mathrm{~min}$ (minor) $] ;{ }^{1} \mathrm{H} \mathrm{NMR}\left(500 \mathrm{MHz}, \mathrm{CDCl}_{3}\right.$ ) $(\delta, \mathrm{ppm}) 7.96-7.91$ (comp, 2H), $7.50(\mathrm{~d}, J=8.4 \mathrm{~Hz}, 2 \mathrm{H}), 7.49-7.45(\mathrm{~m}, 1 \mathrm{H}), 7.42-$ 7.38 (comp, 2H), 7.27 (d, $J=8.4 \mathrm{~Hz}, 2 \mathrm{H}), 5.17$ (d, $J=12.5 \mathrm{~Hz}, 1 \mathrm{H}), 5.14$ (d, $J=12.5$ $\mathrm{Hz}, 1 \mathrm{H}), 3.29-3.21(\mathrm{~m}, 1 \mathrm{H}), 3.19-3.11(\mathrm{~m}, 1 \mathrm{H}), 2.24(\mathrm{t}, J=1.8 \mathrm{~Hz}, 3 \mathrm{H}), 1.43(\mathrm{~s}$, $3 \mathrm{H}), 1.28(\mathrm{~s}, 3 \mathrm{H}) ;{ }^{13} \mathrm{C} \mathrm{NMR}\left(125 \mathrm{MHz}, \mathrm{CDCl}_{3}\right)(\delta, \mathrm{ppm}) 166.5,164.9,160.4,135.5$, $131.8,131.7,130.0,128.5,128.3,127.5,122.3,118.9,101.6,70.7,65.0,34.6,25.7$, 21.8, 14.2; HRMS (ESI) $m / z$ : $[\mathrm{M}+\mathrm{H}]^{+}$Calcd. for $\mathrm{C}_{23} \mathrm{H}_{23} \mathrm{BrNO}_{4}$ 456.0805; Found 456.0793 .<smiles>CC1=C(C(=O)OCc2ccc(C(F)(F)F)cc2)[C@@]2(C)OC(c3ccccc3)=NC2(C)C1</smiles>

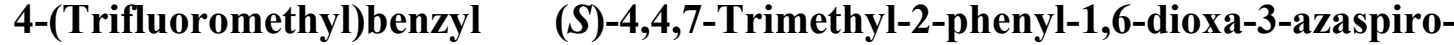
[4.4]nona-2,7-diene-8-carboxylate 4ag

Column chromatoghraphy (silica gel; hexane/ethyl acetate, 5:1); Colorless oil, $59.7 \mathrm{mg}$, 67\% yield, 99\% ee; [HPLC: Chiralpak OD-H column, 3\% IPA in hexane (v/v), 1.0 $\mathrm{mL} / \mathrm{min}, 250 \mathrm{~nm}, \mathrm{t}_{1}=8.0 \mathrm{~min}$ (major), $\mathrm{t}_{2}=6.5 \mathrm{~min}$ (minor) $] ;{ }^{1} \mathrm{H} \mathrm{NMR}\left(500 \mathrm{MHz}, \mathrm{CDCl}_{3}\right.$ ) $(\delta, \mathrm{ppm}) 7.94(\mathrm{~d}, J=7.4 \mathrm{~Hz}, 2 \mathrm{H}), 7.65(\mathrm{~d}, J=7.8 \mathrm{~Hz}, 2 \mathrm{H}), 7.52(\mathrm{~d}, J=7.8 \mathrm{~Hz}, 2 \mathrm{H})$, 7.48 (d, $J=7.5 \mathrm{~Hz}, 1 \mathrm{H}), 7.44-7.37$ (comp, 2H), 5.29 (d, $J=13.1 \mathrm{~Hz}, 1 \mathrm{H}), 5.25$ (d, $J$ $=13.1 \mathrm{~Hz}, 1 \mathrm{H}), 3.26(\mathrm{~d}, J=16.7 \mathrm{~Hz}, 1 \mathrm{H}), 3.17(\mathrm{~d}, J=16.7 \mathrm{~Hz}, 1 \mathrm{H}), 2.26(\mathrm{~s}, 3 \mathrm{H}), 1.44$ $(\mathrm{s}, 3 \mathrm{H}), 1.30(\mathrm{~s}, 3 \mathrm{H}) ;{ }^{13} \mathrm{C} \mathrm{NMR}\left(125 \mathrm{MHz}, \mathrm{CDCl}_{3}\right)(\delta, \mathrm{ppm}) 166.8,164.9,160.5,140.5$, $131.8,130.3$ (q, $J=38.4 \mathrm{~Hz}), 128.5,128.4,128.3,127.5,127.0125 .7$ (q, $J=3.5 \mathrm{~Hz}$ ), $124.2(\mathrm{~d}, J=272.1 \mathrm{~Hz}), 119.0,70.8,64.9,34.6,25.7,21.8,14.3$; HRMS (ESI) $m / z$ : $[\mathrm{M}+\mathrm{H}]^{+}$Calcd. for $\mathrm{C}_{24} \mathrm{H}_{23} \mathrm{~F}_{3} \mathrm{NO}_{4}$ 446.1574; Found 446.1562.<smiles>CCC1=C(C(=O)OC)C[C@@]2(OC(c3ccccc3)=NC2(C)C)C1(C)C</smiles>

Methyl (S)-7-Ethyl-4,4-dimethyl-2-phenyl-1,6-dioxa-3-azaspiro[4.4]nona-2,7diene-8-carboxylate $4 \mathrm{ah}$

Column chromatoghraphy (silica gel; hexane/ethyl acetate, 10:1); Colorless solid, m.p: 99-100 ${ }^{\circ} \mathrm{C}, 48.5 \mathrm{mg}, 77 \%$ yield, 97\% ee; [HPLC: Chiralpak OD-H column, 3\% IPA in hexane $(\mathrm{v} / \mathrm{v}), 1.0 \mathrm{~mL} / \mathrm{min}, 250 \mathrm{~nm}, \mathrm{t}_{1}=6.0 \mathrm{~min}$ (major), $\mathrm{t}_{2}=4.8 \mathrm{~min}($ minor) $] ;{ }^{1} \mathrm{H} \mathrm{NMR}$ $\left(500 \mathrm{MHz}, \mathrm{CDCl}_{3}\right)(\delta, \mathrm{ppm}) 7.96-7.89(\mathrm{comp}, 2 \mathrm{H}), 7.47$ (t, $\left.J=7.4 \mathrm{~Hz}, 1 \mathrm{H}\right), 7.43-$ 7.35 (comp, 2H), 3.74 (s, 3H), 3.22 (d, $J=16.7 \mathrm{~Hz}, 1 \mathrm{H}), 3.13$ (d, $J=16.7 \mathrm{~Hz}, 1 \mathrm{H}), 2.75$ (dq, $J=15.0,7.5 \mathrm{~Hz}, 1 \mathrm{H}), 2.65$ (dq, $J=15.0,7.5 \mathrm{~Hz}, 1 \mathrm{H}), 1.42(\mathrm{~s}, 3 \mathrm{H}), 1.28(\mathrm{~s}, 3 \mathrm{H})$, $1.11(\mathrm{t}, J=7.5 \mathrm{~Hz}, 3 \mathrm{H}) ;{ }^{13} \mathrm{C} \mathrm{NMR}\left(125 \mathrm{MHz}, \mathrm{CDCl}_{3}\right)(\delta, \mathrm{ppm}) 170.5,165.6,160.5$, $131.7,128.5,128.3,127.6,118.7,100.7,70.8,51.2,34.7,25.7,21.6,21.2,11.1$; HRMS (ESI) $m / z:[\mathrm{M}+\mathrm{H}]^{+}$Calcd. for $\mathrm{C}_{18} \mathrm{H}_{22} \mathrm{NO}_{4}$ 316.1543; Found 316.1536 . 
<smiles>COC(=O)C1=C(C(=O)c2ccccc2)OC2(OC(c3ccccc3)=NC2(C)C)O1</smiles>

Methyl (S)-7-Butyl-4,4-dimethyl-2-phenyl-1,6-dioxa-3-azaspiro[4.4]nona-2,7diene-8-carboxylate 4ai

Column chromatoghraphy (silica gel; hexane/ethyl acetate, 10:1); Colorless oil, 49.4 mg, 75\% yield, 96\% ee; [HPLC: Chiralpak OD-H column, 3\% IPA in hexane (v/v), 1.0 $\mathrm{mL} / \mathrm{min}, 250 \mathrm{~nm}, \mathrm{t}_{1}=5.6 \mathrm{~min}$ (major), $\mathrm{t}_{2}=4.6 \mathrm{~min}$ (minor) $] ;{ }^{1} \mathrm{H} \mathrm{NMR}\left(500 \mathrm{MHz}, \mathrm{CDCl}_{3}\right.$ ) ( $\delta$, ppm) $7.95-7.90$ (comp, 2H), $7.50-7.45$ (m, 1H), $7.43-7.36$ (comp, 2H), 3.75 (s, $3 \mathrm{H}), 3.23(\mathrm{~d}, J=16.7 \mathrm{~Hz}, 1 \mathrm{H}), 3.13(\mathrm{~d}, J=16.7 \mathrm{~Hz}, 1 \mathrm{H}), 2.74-2.60$ (comp, 2H), 1.65 - 1.52 (comp, 2H), 1.43 (s, 3H), 1.29 (s, 3H), 0.93 (t, $J=7.4 \mathrm{~Hz}, 3 \mathrm{H}) ;{ }^{13} \mathrm{C}$ NMR (125 $\left.\mathrm{MHz}, \mathrm{CDCl}_{3}\right)(\delta, \mathrm{ppm}) 169.4,165.7,160.5,131.7,128.5,128.3,127.6,118.7,101.6$, 70.7, 51.2, 34.7, 29.4, 25.7, 21.7, 20.1, 13.7; HRMS (ESI) $m / z:[\mathrm{M}+\mathrm{H}]^{+}$Calcd. for $\mathrm{C}_{19} \mathrm{H}_{24} \mathrm{NO}_{4} 330.1700$; Found 330.1690.<smiles>CC(=O)C1=C(CBr)O[C@]2(C1)OC(c1ccccc1)=NC2(C)C</smiles>

Methyl (S)-4,4-Dimethyl-7-phenethyl-2-phenyl-1,6-dioxa-3-azaspiro[4.4]nona2,7-diene-8-carboxylate 4aj

Column chromatoghraphy (silica gel; hexane/ethyl acetate, 10:1); Colorless oil, 61.0 mg, 78\% yield, 95\% ee; [HPLC: Chiralpak OD-H column, 3\% IPA in hexane (v/v), 1.0 $\mathrm{mL} / \mathrm{min}, 250 \mathrm{~nm}, \mathrm{t}_{1}=7.5 \mathrm{~min}$ (major), $\mathrm{t}_{2}=6.8 \mathrm{~min}$ (minor) $] ;{ }^{1} \mathrm{H} \mathrm{NMR}\left(500 \mathrm{MHz}, \mathrm{CDCl}_{3}\right.$ ) ( $\delta$, ppm) $7.98-7.91$ (comp, 2H), 7.49 (t, $J=7.4 \mathrm{~Hz}, 1 \mathrm{H}), 7.45-7.39$ (comp, 2H), 7.27 - 7.22 (comp, 2H), 7.22 - 7.14 (comp, 3H), 3.73 (s, 3H), 3.21 (d, $J=16.8 \mathrm{~Hz}, 1 \mathrm{H})$, $3.13(\mathrm{~d}, J=16.8 \mathrm{~Hz}, 1 \mathrm{H}), 3.09-2.96$ (comp, 2H), $2.94-2.80$ (comp, 2H), 1.41 (s, 3H), 1.29 (s, 3H); ${ }^{13} \mathrm{C}$ NMR (125 MHz, $\left.\mathrm{CDCl}_{3}\right)(\delta, \mathrm{ppm}) 168.1,165.4,160.5,140.6,131.7$, 128.5, 128.5, 128.4, 128.3, 127.6, 126.2, 118.9, 102.0, 70.7, 51.2, 34.7, 32.7, 29.4, 25.7, 21.7; HRMS (ESI) $m / z$ : $[\mathrm{M}+\mathrm{H}]^{+}$Calcd. for $\mathrm{C}_{24} \mathrm{H}_{26} \mathrm{NO}_{4}$ 392.1856; Found 392.1856.<smiles>COc1ccc(C2=NC(C)(C)[C@]3(O2)OC(C)=C(C(C)=O)O3)cc1</smiles>

Methyl (S)-2-(4-Methoxyphenyl)-4,4,7-trimethyl-1,6-dioxa-3-azaspiro[4.4]nona2,7-diene-8-carboxylate $4 \mathrm{ba}$

Column chromatoghraphy (silica gel; hexane/ethyl acetate, 5:1); Colorless oil, $48.3 \mathrm{mg}$, 73\% yield, 98\% ee; [HPLC: Chiralpak OD-H column, 3\% IPA in hexane (v/v), 1.0 $\mathrm{mL} / \mathrm{min}, 250 \mathrm{~nm}, \mathrm{t}_{1}=15.2 \min$ (major), $\mathrm{t}_{2}=8.0 \mathrm{~min}$ (minor)]; ${ }^{1} \mathrm{H}$ NMR (500 MHz, $\left.\mathrm{CDCl}_{3}\right)(\delta, \mathrm{ppm}) 7.86(\mathrm{~d}, J=8.9 \mathrm{~Hz}, 2 \mathrm{H}), 6.88(\mathrm{~d}, J=8.9 \mathrm{~Hz}, 2 \mathrm{H}), 3.82(\mathrm{~s}, 3 \mathrm{H}), 3.74$ 
(s, 3H), $3.25-3.17(\mathrm{~m}, 1 \mathrm{H}), 3.15-3.08(\mathrm{~m}, 1 \mathrm{H}), 2.22(\mathrm{t}, J=1.6 \mathrm{~Hz}, 3 \mathrm{H}), 1.40(\mathrm{~s}, 3 \mathrm{H})$, $1.26(\mathrm{~s}, 3 \mathrm{H}) ;{ }^{13} \mathrm{C} \mathrm{NMR}\left(125 \mathrm{MHz}, \mathrm{CDCl}_{3}\right)(\delta, \mathrm{ppm}) 165.9,165.7,162.4,160.2,130.1$, $119.9,118.8,113.8,101.8,70.5,55.5,51.2,34.6,25.7,21.8,14.1$; HRMS (ESI) $m / z$ : $[\mathrm{M}+\mathrm{H}]^{+}$Calcd. for $\mathrm{C}_{18} \mathrm{H}_{22} \mathrm{NO}_{5}$ 332.1492; Found 332.1480.<smiles>COC(=O)C1=C(C)O[C@]2(C1)OC(c1ccc(C(F)(F)F)cc1)=NC2(C)C</smiles>

Methyl

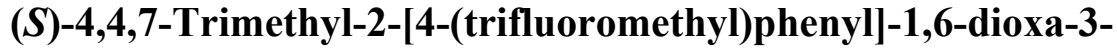
azaspiro[4.4]nona-2,7-diene-8-carboxylate 4ca

Column chromatoghraphy (silica gel; hexane/ethyl acetate, 5:1); Colorless oil, $56.1 \mathrm{mg}$, 76\% yield, 98\% ee; [HPLC: Chiralpak OD-H column, 3\% IPA in hexane (v/v), 1.0 $\mathrm{mL} / \mathrm{min}, 250 \mathrm{~nm}, \mathrm{t}_{1}=12.2 \min$ (major), $\mathrm{t}_{2}=11.1 \min$ (minor) $] ;{ }^{1} \mathrm{H}$ NMR $(500 \mathrm{MHz}$, $\left.\mathrm{CDCl}_{3}\right)(\delta, \mathrm{ppm}) 8.05(\mathrm{~d}, J=8.1 \mathrm{~Hz}, 2 \mathrm{H}), 7.66(\mathrm{~d}, J=8.1 \mathrm{~Hz}, 2 \mathrm{H}), 3.76(\mathrm{~s}, 3 \mathrm{H}), 3.27-$ $3.20(\mathrm{~m}, 1 \mathrm{H}), 3.17-3.10(\mathrm{~m}, 1 \mathrm{H}), 2.24(\mathrm{t}, J=1.8 \mathrm{~Hz}, 3 \mathrm{H}), 1.43(\mathrm{~s}, 3 \mathrm{H}), 1.29(\mathrm{~s}, 3 \mathrm{H})$; ${ }^{13} \mathrm{C}$ NMR $\left(125 \mathrm{MHz}, \mathrm{CDCl}_{3}\right)(\delta, \mathrm{ppm}) 165.7,165.6,159.4,133.3$ (q, $J=32.7 \mathrm{~Hz}$ ), 130.9, 128.7, $125.6(\mathrm{q}, J=3.7 \mathrm{~Hz}), 123.9$ (q, $J=272.6 \mathrm{~Hz}), 119.1,102.0,71,51.3$, 34.8, 25.6, 21.7, 14.1; HRMS (ESI) $m / z$ : $[\mathrm{M}+\mathrm{H}]^{+}$Calcd. for $\mathrm{C}_{18} \mathrm{H}_{19} \mathrm{~F}_{3} \mathrm{NO}_{4} 370.1261$; Found 370.1252 .<smiles></smiles>

Methyl (S)-2-(4-Bromophenyl)-4,4,7-trimethyl-1,6-dioxa-3-azaspiro[4.4]nona-2,7diene-8-carboxylate $4 \mathrm{da}$

Column chromatoghraphy (silica gel; hexane/ethyl acetate, 10:1); Colorless oil, 56.9 mg, 75\% yield, 96\% ee; [HPLC: Chiralpak OD-H column, 3\% IPA in hexane (v/v), 1.0 $\mathrm{mL} / \mathrm{min}, 250 \mathrm{~nm}, \mathrm{t}_{1}=5.9 \mathrm{~min}$ (major), $\mathrm{t}_{2}=5.2 \mathrm{~min}$ (minor) $] ;{ }^{1} \mathrm{H} \mathrm{NMR}\left(500 \mathrm{MHz}, \mathrm{CDCl}_{3}\right)$ $(\delta, \mathrm{ppm}) 7.78(\mathrm{~d}, J=8.3 \mathrm{~Hz}, 2 \mathrm{H}), 7.52(\mathrm{~d}, J=8.3 \mathrm{~Hz}, 2 \mathrm{H}), 3.74(\mathrm{~s}, 3 \mathrm{H}), 3.24-3.16$ $(\mathrm{m}, 1 \mathrm{H}), 3.14-3.07(\mathrm{~m}, 1 \mathrm{H}), 2.22(\mathrm{~s}, 3 \mathrm{H}), 1.40(\mathrm{~s}, 3 \mathrm{H}), 1.26(\mathrm{~s}, 3 \mathrm{H}) ;{ }^{13} \mathrm{C}$ NMR $(125$ $\left.\mathrm{MHz}, \mathrm{CDCl}_{3}\right)(\delta, \mathrm{ppm}) 165.7,165.6,159.7,131.7,129.8,126.5,126.4,119.0,101.9$, 70.8, 51.2, 34.7, 25.6, 21.7, 14.0; HRMS (ESI) $m / z$ : $[\mathrm{M}+\mathrm{H}]^{+}$Calcd. for $\mathrm{C}_{17} \mathrm{H}_{19} \mathrm{~B}_{\mathrm{r}} \mathrm{NO}_{4}$ 380.0492; Found 380.0492 .<smiles>CC(=O)C1=C(C)O[C@@]2(C1)OC(c1ccccc1Br)=NC2(C)C</smiles>

Methyl (S)-2-(2-Bromophenyl)-4,4,7-trimethyl-1,6-dioxa-3-azaspiro[4.4]nona-2,7- 


\section{diene-8-carboxylate 4ea}

Column chromatoghraphy (silica gel; hexane/ethyl acetate, 10:1); Colorless oil, 56.9 mg, 75\% yield, 95.5\% ee; [HPLC: Chiralpak OD-H column, 3\% IPA in hexane (v/v), $1.0 \mathrm{~mL} / \mathrm{min}, 250 \mathrm{~nm}, \mathrm{t}_{1}=9.6 \mathrm{~min}$ (major), $\mathrm{t}_{2}=8.0 \mathrm{~min}$ (minor) $] ;{ }^{1} \mathrm{H} \mathrm{NMR}(500 \mathrm{MHz}$, $\left.\mathrm{CDCl}_{3}\right)(\delta, \mathrm{ppm}) 7.66-7.63(\mathrm{~m}, 1 \mathrm{H}), 7.63-7.59(\mathrm{~m}, 1 \mathrm{H}), 7.35-7.30(\mathrm{~m}, 1 \mathrm{H}), 7.30-$ $7.25(\mathrm{~m}, 1 \mathrm{H}), 3.73(\mathrm{~s}, 3 \mathrm{H}), 3.25-3.18(\mathrm{~m}, 1 \mathrm{H}), 3.15-3.08(\mathrm{~m}, 1 \mathrm{H}), 2.25(\mathrm{t}, J=1.9$ $\mathrm{Hz}, 3 \mathrm{H}), 1.44$ (s, 3H), 1.33 (s, 3H); ${ }^{13} \mathrm{C} \mathrm{NMR}\left(125 \mathrm{MHz}, \mathrm{CDCl}_{3}\right)(\delta, \mathrm{ppm}) 165.8,165.6$, $159.9,133.9,132.0,131.5,129.4,127.2,122.0,119.1,102.0,71.0,51.2,34.6,25.5$, 21.5, 14.1; HRMS (ESI) $m / z$ : $[\mathrm{M}+\mathrm{H}]^{+}$Calcd. for $\mathrm{C}_{17} \mathrm{H}_{19} \mathrm{~B}_{\mathrm{r}} \mathrm{NO}_{4}$ 380.0492; Found 380.0492 .<smiles>CCOC(C)=C(C)O[C@]1(C)OC(c2ccc(Br)cc2Cl)=NC1(C)C</smiles>

\section{Methyl (S)-2-(4-Bromo-2-chlorophenyl)-4,4,7-trimethyl-1,6-dioxa-3-azaspiro-} [4.4]nona-2,7-diene-8-carboxylate 4 fa

Column chromatoghraphy (silica gel; hexane/ethyl acetate, 10:1); Colorless oil, 66.9 mg, 81\% yield, 96\% ee; [HPLC: Chiralpak OD-H column, 3\% IPA in hexane (v/v), 1.0 $\mathrm{mL} / \mathrm{min}, 250 \mathrm{~nm}, \mathrm{t}_{1}=7.0 \mathrm{~min}$ (major), $\mathrm{t}_{2}=6.4 \mathrm{~min}$ (minor) $] ;{ }^{1} \mathrm{H} \mathrm{NMR}\left(500 \mathrm{MHz}, \mathrm{CDCl}_{3}\right)$ $(\delta, \mathrm{ppm}) 7.61-7.56(\mathrm{comp}, 2 \mathrm{H}), 7.43-7.38(\mathrm{~m}, 1 \mathrm{H}), 3.72(\mathrm{~s}, 3 \mathrm{H}), 3.25-3.17(\mathrm{~m}$, $1 \mathrm{H}), 3.13-3.05(\mathrm{~m}, 1 \mathrm{H}), 2.23(\mathrm{~s}, 3 \mathrm{H}), 1.41(\mathrm{~s}, 3 \mathrm{H}), 1.29(\mathrm{~s}, 3 \mathrm{H}) ;{ }^{13} \mathrm{C}$ NMR $(125 \mathrm{MHz}$, $\left.\mathrm{CDCl}_{3}\right)(\delta, \mathrm{ppm}) 165.7,165.5,158.4,134.7,133.5,132.4,129.9,126.1,125.4,118.9$, 101.9, 71.1, 51.2, 34.6, 25.5, 21.4, 14.0; HRMS (ESI) $\mathrm{m} / \mathrm{z}:[\mathrm{M}+\mathrm{H}]^{+}$Calcd. for $\mathrm{C}_{17} \mathrm{H}_{18} \mathrm{~B}_{\mathrm{r}} \mathrm{ClNO}_{4}$ 414.0102; Found 414.0102.<smiles>CC(=O)C1=C(C)O[C@@]2(OC(c3cccc4ccccc34)=NC2(C)C)O1</smiles>

Methyl (S)-4,4,7-Trimethyl-2-(naphthalen-1-yl)-1,6-dioxa-3-azaspiro[4.4]nona2,7-diene-8-carboxylate 4ga

Column chromatoghraphy (silica gel; hexane/ethyl acetate, 10:1); Colorless oil, 55.5 mg, 79\% yield, 97\% ee; [HPLC: Chiralpak OD-H column, 3\% IPA in hexane (v/v), 1.0 $\mathrm{mL} / \mathrm{min}, 250 \mathrm{~nm}, \mathrm{t}_{1}=7.4 \mathrm{~min}$ (major), $\mathrm{t}_{2}=6.8 \mathrm{~min}$ (minor) $] ;{ }^{1} \mathrm{H} \mathrm{NMR}\left(500 \mathrm{MHz}, \mathrm{CDCl}_{3}\right)$ $(\delta, \mathrm{ppm}) 9.09(\mathrm{~d}, J=8.6 \mathrm{~Hz}, 1 \mathrm{H}), 8.08-8.02(\mathrm{~m}, 1 \mathrm{H}), 7.95(\mathrm{~d}, J=8.2 \mathrm{~Hz}, 1 \mathrm{H}), 7.86$ $(\mathrm{d}, J=8.1 \mathrm{~Hz}, 1 \mathrm{H}), 7.63-7.57(\mathrm{~m}, 1 \mathrm{H}), 7.55-7.44(\mathrm{comp}, 2 \mathrm{H}), 3.77(\mathrm{~s}, 3 \mathrm{H}), 3.32-$ $3.25(\mathrm{~m}, 1 \mathrm{H}), 3.23-3.16(\mathrm{~m}, 1 \mathrm{H}), 2.30(\mathrm{t}, J=1.8 \mathrm{~Hz}, 3 \mathrm{H}), 1.53(\mathrm{~s}, 3 \mathrm{H}), 1.40(\mathrm{~s}, 3 \mathrm{H})$; ${ }^{13} \mathrm{C} \mathrm{NMR}\left(125 \mathrm{MHz}, \mathrm{CDCl}_{3}\right)(\delta, \mathrm{ppm}) 165.9,165.7,160.3,133.8,132.2,131.2,129.2$, 128.5, 127.6, 126.3, 126.3, 124.7, 124.2, 118.1, 101.9, 71.4, 51.2, 34.5, 25.8, 21.8, 14.1; HRMS (ESI) $m / z$ : [M+H] $]^{+}$Calcd. for $\mathrm{C}_{21} \mathrm{H}_{22} \mathrm{NO}_{4}$ 352.1543; Found 352.1543. 
<smiles>CC(=O)C1=C(C)O[C@]2(C1)OC(c1ccco1)=NC2(C)C</smiles>

Methyl (S)-2-(Furan-2-yl)-4,4,7-trimethyl-1,6-dioxa-3-azaspiro[4.4]nona-2,7diene-8-carboxylate 4 ha

Column chromatoghraphy (silica gel; hexane/ethyl acetate, 10:1); Colorless oil, 42.5 mg, 73\% yield, 98\% ee; [HPLC: Chiralpak OD-H column, 3\% IPA in hexane (v/v), 1.0 $\mathrm{mL} / \mathrm{min}, 250 \mathrm{~nm}, \mathrm{t}_{1}=10.5 \min$ (major), $\mathrm{t}_{2}=9.9 \min$ (minor) $] ;{ }^{1} \mathrm{H}$ NMR (500 MHz, $\left.\mathrm{CDCl}_{3}\right)(\delta, \mathrm{ppm}) 7.57-7.52(\mathrm{~m}, 1 \mathrm{H}), 6.99-6.94(\mathrm{~m}, 1 \mathrm{H}), 6.52-6.46(\mathrm{~m}, 1 \mathrm{H}), 3.74$ $(\mathrm{s}, 3 \mathrm{H}), 3.25-3.18(\mathrm{~m}, 1 \mathrm{H}), 3.17-3.08(\mathrm{~m}, 1 \mathrm{H}), 2.24(\mathrm{t}, J=1.9 \mathrm{~Hz}, 3 \mathrm{H}), 1.42(\mathrm{~s}, 3 \mathrm{H})$, 1.29 (s, 3H); ${ }^{13} \mathrm{C}$ NMR $\left(125 \mathrm{MHz}, \mathrm{CDCl}_{3}\right)(\delta, \mathrm{ppm}) 165.7,165.5,152.9,145.6,142.6$, $118.9,115.0,111.7,101.9,70.5,51.2,34.7,25.6,21.7,14.0$; HRMS (ESI) $m / z:[\mathrm{M}+\mathrm{H}]^{+}$ Calcd. for $\mathrm{C}_{15} \mathrm{H}_{18} \mathrm{NO}_{5}$ 292.1179; Found 292.1178.<smiles>CCOC(C)=C(C)OC12OC(/C=C/c3ccccc3)=NC1(C)C2(C)C</smiles>

Methyl (S, E)-4,4,7-Trimethyl-2-styryl-1,6-dioxa-3-azaspiro[4.4]nona-2,7-diene-8carboxylate 4ia

Column chromatoghraphy (silica gel; hexane/ethyl acetate, 10:1); Colorless oil, 53.7 mg, 82\% yield, 97\% ee; [HPLC: Chiralpak OD-H column, 3\% IPA in hexane (v/v), 1.0 $\mathrm{mL} / \mathrm{min}, 250 \mathrm{~nm}, \mathrm{t}_{1}=14.0 \min$ (major), $\mathrm{t}_{2}=7.0 \min ($ minor) $) ;{ }^{1} \mathrm{H}$ NMR $(500 \mathrm{MHz}$, $\left.\mathrm{CDCl}_{3}\right)(\delta, \mathrm{ppm}) 7.49-7.45$ (comp, 2H), $7.38-7.32$ (comp, 4H), 6.58 (d, $J=16.3 \mathrm{~Hz}$, $1 \mathrm{H}), 3.75(\mathrm{~s}, 3 \mathrm{H}), 3.23-3.17(\mathrm{~m}, 1 \mathrm{H}), 3.15-3.06(\mathrm{~m}, 1 \mathrm{H}), 2.25(\mathrm{t}, J=1.8 \mathrm{~Hz}, 3 \mathrm{H})$, $1.38(\mathrm{~s}, 3 \mathrm{H}), 1.25(\mathrm{~s}, 3 \mathrm{H}) ;{ }^{13} \mathrm{C} \mathrm{NMR}\left(125 \mathrm{MHz}, \mathrm{CDCl}_{3}\right)(\delta, \mathrm{ppm}) 165.9,165.7,160.3$, 140.7, 135.2, 129.7, 129.0, 127.6, 118.6, 115.0, 101.8, 70.4, 51.2, 34.7, 25.7, 21.7, 14.1; HRMS (ESI) $m / z$ : [M+H] ${ }^{+}$Calcd. for $\mathrm{C}_{19} \mathrm{H}_{22} \mathrm{NO}_{4} 328.1543$; Found 328.1543.<smiles>COC(=O)C1=C(C)O[C@@]2(OC(C(C)(C)C)=NC2(C)C)O1</smiles>

Methyl (S)-2-(tert-Butyl)-4,4,7-trimethyl-1,6-dioxa-3-azaspiro[4.4]nona-2,7-diene8-carboxylate 4 ja

Column chromatoghraphy (silica gel; hexane/ethyl acetate, 5:1); Colorless oil, $29.8 \mathrm{mg}$, 53\% yield, 96\% ee; [HPLC: Chiralpak OD-H column, 3\% IPA in hexane (v/v), 1.0 $\mathrm{mL} / \mathrm{min}, 250 \mathrm{~nm}, \mathrm{t}_{1}=10.7 \min$ (major), $\mathrm{t}_{2}=9.5 \min ($ minor) $) ;{ }^{1} \mathrm{H}$ NMR $(500 \mathrm{MHz}$, $\left.\mathrm{CDCl}_{3}\right)(\delta, \mathrm{ppm}) 3.73(\mathrm{~s}, 3 \mathrm{H}), 3.14-3.08(\mathrm{~m}, 1 \mathrm{H}), 3.02-2.94(\mathrm{~m}, 1 \mathrm{H}), 2.21(\mathrm{t}, J=1.7$ $\mathrm{Hz}, 3 \mathrm{H}), 1.30$ (s, 3H), 1.19 (s, 9H), 1.14 (s, 3H); ${ }^{13} \mathrm{C} \mathrm{NMR}\left(125 \mathrm{MHz}, \mathrm{CDCl}_{3}\right)(\delta, \mathrm{ppm})$ 170.6, 165.9, 165.8, 118.6, 101.6, 69.9, 51.2, 34.3, 33.0, 27.4, 25.6, 21.4, 14.1; HRMS 
(ESI) $m / z:[\mathrm{M}+\mathrm{H}]^{+}$Calcd. for $\mathrm{C}_{15} \mathrm{H}_{24} \mathrm{NO}_{4}$ 282.1700; Found 282.1700.<smiles>CC(=O)C1=C(C)O[C@]2(CCCc3ccccc32)C1</smiles>

Methyl (R)-5-Methyl-3',4'-dihydro-2' $H, 3 H$-spiro[furan-2, 1'-naphthalene]-4carboxylate 4la

Column chromatoghraphy (silica gel; hexane/ethyl acetate, 20:1); Colorless oil, 34.1 mg, 66\% yield, 94\% ee; [HPLC: Chiralpak OD-H column, 1\% IPA in hexane (v/v), $0.75 \mathrm{~mL} / \mathrm{min}, 250 \mathrm{~nm}, \mathrm{t}_{1}=5.2 \mathrm{~min}$ (major), $\mathrm{t}_{2}=6.6 \min ($ minor $\left.)\right] ;{ }^{1} \mathrm{H}$ NMR $(500 \mathrm{MHz}$, $\left.\mathrm{CDCl}_{3}\right)(\delta, \mathrm{ppm}) 7.39-7.33(\mathrm{~m}, 1 \mathrm{H}), 7.24-7.16$ (comp, 2H), $7.13-7.04(\mathrm{~m}, 1 \mathrm{H})$, $3.73(\mathrm{~s}, 3 \mathrm{H}), 3.17$ (dd, $J=14.8,1.8 \mathrm{~Hz}, 1 \mathrm{H}), 2.99(\mathrm{dd}, J=14.8,1.8 \mathrm{~Hz}, 1 \mathrm{H}), 2.90-$ 2.75 (comp, 2H), 2.27 (t, $J=1.6 \mathrm{~Hz}, 3 \mathrm{H}), 2.16-2.08$ (m, 1H), $2.04-1.94$ (comp, 2H), $1.90-1.79(\mathrm{~m}, 1 \mathrm{H}) ;{ }^{13} \mathrm{C} \mathrm{NMR}\left(125 \mathrm{MHz}, \mathrm{CDCl}_{3}\right)(\delta, \mathrm{ppm}) 167.4,166.8,139.8,136.5$, $128.9,128.0,126.8,126.5,100.6,87.2,50.9,45.0,36.9,29.5,19.9,14.5$; HRMS (ESI) $m / z:[\mathrm{M}+\mathrm{Na}]^{+}$Calcd. for $\mathrm{C}_{16} \mathrm{H}_{18} \mathrm{NaO}_{3}$ 281.1148; Found 281.1146.<smiles>COC(=O)C1(C(=O)c2ccccc2)CC12OC(c1ccccc1)=NC2(C)C</smiles>

Methyl (1R, 3R)-1-Benzoyl-7,7-dimethyl-5-phenyl-4-oxa-6-azaspiro[2.4]hept-5ene-1-carboxylate 6ab

Column chromatoghraphy (silica gel; hexane/ethyl acetate, 10:1); Colorless oil, 63.2 mg, 87\% yield, 98\% ee; [HPLC: Chiralpak OD-H column, 3\% IPA in hexane (v/v), 1.2 $\mathrm{mL} / \mathrm{min}, 250 \mathrm{~nm}, \mathrm{t}_{1}=16.5 \min$ (major), $\mathrm{t}_{2}=15.2 \min$ (minor) $) ;{ }^{1} \mathrm{H}$ NMR $(500 \mathrm{MHz}$, $\left.\mathrm{CDCl}_{3}\right)(\delta, \mathrm{ppm}) 8.01-7.95$ (comp, 2H), 7.68 - 7.62 (comp, 2H), $7.54-7.48(\mathrm{~m}, 1 \mathrm{H})$, 7.47 - 7.41 (comp, 3H), 7.40 - 7.35 (comp, 2H), 3.74 (s, 3H), 3.52 (d, J=15.7 Hz, 1H), $3.38(\mathrm{~d}, J=15.7 \mathrm{~Hz}, 1 \mathrm{H}), 1.50(\mathrm{~s}, 3 \mathrm{H}), 1.33(\mathrm{~s}, 3 \mathrm{H}) ;{ }^{13} \mathrm{C} \mathrm{NMR}\left(125 \mathrm{MHz}, \mathrm{CDCl}_{3}\right)(\delta$, ppm) 190.0, 164.2, 160.4, 140.3, 132.0, 130.7, 128.6, 128.3, 128.1, 127.7, 127.1, 117.9, 87.9, 71.5, 57.3, 34.2, 25.1, 21.7; HRMS (ESI) $m / z$ : $[\mathrm{M}+\mathrm{H}]^{+}$Calcd. for $\mathrm{C}_{22} \mathrm{H}_{22} \mathrm{NO}_{4}$ 364.1543; Found 364.1543.<smiles>CC1(C)N=C(c2ccccc2)OC12CC2(C)C(=O)c1ccccc1</smiles>

\section{Phenyl(1S,3R)-1,7,7-trimethyl-5-phenyl-4-oxa-6-azaspiro[2.4]hept-5-en-1-yl-} methanone 6ad

Column chromatoghraphy (silica gel; hexane/ethyl acetate, 10:1); Colorless oil, 56.2 $\mathrm{mg}, 88 \%$ yield, 99\% ee; [HPLC: (R,R-Whelk-O1) column, 2\% IPA in hexane (v/v), $0.75 \mathrm{~mL} / \mathrm{min}, 250 \mathrm{~nm}, \mathrm{t}_{1}=21.7 \mathrm{~min}$ (major), $\mathrm{t}_{2}=14.4 \mathrm{~min}$ (minor) $) ;{ }^{1} \mathrm{H} \mathrm{NMR}(500 \mathrm{MHz}$, $\left.\mathrm{CDCl}_{3}\right)(\delta, \mathrm{ppm}) 8.00-7.95$ (comp, 2H), 7.84 - 7.79 (comp, 2H), 7.54 - 7.50 (comp, 
2H), $7.48-7.42$ (comp, 4H), 2.30 (d, $J=6.4 \mathrm{~Hz}, 1 \mathrm{H}), 1.71$ (s, 3H), 1.39 (s, 3H), 1.13 (s, 3H), 1.06 (d, $J=6.4 \mathrm{~Hz}, 1 \mathrm{H}) ;{ }^{13} \mathrm{C} \mathrm{NMR}\left(125 \mathrm{MHz}, \mathrm{CDCl}_{3}\right)(\delta, \mathrm{ppm}) 201.5,160.7$, $137.8,132.3,131.6,129.2$, 128.5, 128.4, 128.3, 128.2, 127.8, 82.7, 68.4, 34.3, 28.1, 27.0, 21.8, 20.4; HRMS (ESI) $m / z$ : $[\mathrm{M}+\mathrm{H}]^{+}$Calcd. for $\mathrm{C}_{21} \mathrm{H}_{22} \mathrm{NO}_{2}$ 320.1645; Found 320.1645 .<smiles>COC(=O)C1=C(c2ccccc2)O[C@]2(C1)OC(c1ccccc1)=NC2(C)C</smiles>

Methyl (S)-4,4-Dimethyl-2,7-diphenyl-1,6-dioxa-3-azaspiro[4.4]nona-2,7-diene-8carboxylate 7 ab

Column chromatoghraphy (silica gel; hexane/ethyl acetate, 10:1); Colorless oil, $5.1 \mathrm{mg}$, $7 \%$ yield, 25\% ee; ${ }^{1} \mathrm{H}$ NMR (500 MHz, $\left.\mathrm{CDCl}_{3}\right)(\delta$, ppm) $7.99-7.93$ (comp, 2H), 7.89 - 7.83 (comp, 2H), $7.51-7.45(\mathrm{~m}, 1 \mathrm{H}), 7.44-7.35$ (comp, 5H), 3.75 (s, 3H), 3.50 (d, $J=17.3 \mathrm{~Hz}, 1 \mathrm{H}), 3.37$ (d, $J=17.3 \mathrm{~Hz}, 1 \mathrm{H}), 1.53(\mathrm{~s}, 3 \mathrm{H}), 1.36(\mathrm{~s}, 3 \mathrm{H}) ;{ }^{13} \mathrm{C}$ NMR $(125$ $\left.\mathrm{MHz}, \mathrm{CDCl}_{3}\right)(\delta, \mathrm{ppm}) 165.0,162.7,160.5,131.8,130.9,129.5,129.2,128.5,128.4$, $127.9,127.6,118.1,102.0,70.9,51.5,36.7,25.8,22.0$; HRMS (ESI) $m / z:[\mathrm{M}+\mathrm{H}]^{+}$ Calcd. for $\mathrm{C}_{22} \mathrm{H}_{22} \mathrm{NO}_{4}$ 364.1543; Found 364.1543.<smiles>CC(C)=C1COC2(C1)OC(c1ccccc1)=NC2(C)C</smiles>

Phenyl (S)-(4,4,7-Trimethyl-2-phenyl-1,6-dioxa-3-azaspiro[4.4]nona-2,7-dien-8yl)methanone 7 ac

Column chromatoghraphy (silica gel; hexane/ethyl acetate, 10:1); Colorless oil, 64.6 mg, 93\% yield, 0\% ee; ${ }^{1} \mathrm{H} \mathrm{NMR}\left(500 \mathrm{MHz}, \mathrm{CDCl}_{3}\right)(\delta$, ppm) $7.70-7.63$ (comp, 2H), $7.46-7.39$ (comp, 5H), $7.39-7.32$ (comp, 3H), 4.48 (d, $J=3.3 \mathrm{~Hz}, 1 \mathrm{H}), 4.08$ (d, $J=$ $3.3 \mathrm{~Hz}, 1 \mathrm{H}), 1.86(\mathrm{~s}, 3 \mathrm{H}), 1.84(\mathrm{~s}, 3 \mathrm{H}), 1.70(\mathrm{~s}, 3 \mathrm{H}) ;{ }^{13} \mathrm{C} \mathrm{NMR}\left(125 \mathrm{MHz}, \mathrm{CDCl}_{3}\right)(\delta$, ppm) $164.4,163.5,155.3,138.0,132.4,130.3,130.0,129.2,128.5,128.2,126.1,111.9$, 109.0, 81.9, 77.4, 77.2, 76.9, 63.2, 27.7, 26.8, 11.8; HRMS (ESI) $m / z:[\mathrm{M}+\mathrm{H}]^{+}$Calcd. for $\mathrm{C}_{22} \mathrm{H}_{22} \mathrm{NO}_{3}$ 348.1594; Found 348.1594.<smiles>CC(=O)C(Cc1cnc(-c2ccccc2)o1)C(C)=O</smiles>

\section{Methyl 3-Oxo-2-(2-phenyloxazol-5-ylmethyl)butanoate 8ka}

Column chromatoghraphy (silica gel; hexane/ethyl acetate, 10:1); Colorless oil, 42.6 mg, 78\% yield, ${ }^{1} \mathrm{H}$ NMR (500 MHz, $\left.\mathrm{CDCl}_{3}\right)(\delta$, ppm) $8.00-7.94(\mathrm{comp}, 2 \mathrm{H}), 7.46-$ 7.42 (comp, 3H), 6.89 (s, 1H), 3.91 (t, $J=7.4 \mathrm{~Hz}, 1 \mathrm{H}), 3.77$ (s, 3H), 3.36-3.25 (comp, 2H), $2.30(\mathrm{~s}, 3 \mathrm{H}) ;{ }^{13} \mathrm{C} \mathrm{NMR}\left(125 \mathrm{MHz}, \mathrm{CDCl}_{3}\right)(\delta, \mathrm{ppm})$ 201.0, 169.0, 161.4, 149.1, $130.4,128.9,127.5,126.2,125.4,57.9,53.0,29.6,24.3$.; HRMS (ESI) $m / z:[\mathrm{M}+\mathrm{H}]^{+}$ 
Calcd. for $\mathrm{C}_{15} \mathrm{H}_{16} \mathrm{NO}_{4}$ 274.1074; Found 274.1077.

3.2 General Procedure for Gram Scale Reaction of Spiroketal 4 from 1a and 2a.<smiles>C=C1OC(c2ccccc2)=NC1(C)C</smiles><smiles>C=C(OCC)C(=[18O])C(=O)OC</smiles>

(1) $\mathrm{Rh}_{2}(S-\mathrm{TCPTTL})_{4}(0.5 \mathrm{~mol} \%)$

(2) TABF (2.0 eq), DCM, $5 \mathrm{~min}$<smiles>CC(=O)C1=C(C)O[C@@]2(OC(c3ccccc3)=NC2(C)C)C1(C)C</smiles>

To a 50-mL oven-dried flask containing a magnetic stirring bar were added $\mathrm{Rh}_{2}(S$ TCPTTL) 4 (36 mg, $0.5 \mathrm{~mol} \%$ ) and 5-methylene-4,5-dihydrooxazole 1 (4.0 mmol) in dry DCM $(20.0 \mathrm{~mL})$. Then enoldiazo compound $2(6.0 \mathrm{mmol})$ in dry DCM $(15.0 \mathrm{~mL})$ was added as a solution via syringe pump over $1 \mathrm{~h}$ under an argon atmosphere at room temperature. After addition, the reaction solution was stirred overnight under these conditions until consumption of the reactants (monitored by TLC) was complete. Then the reaction vial was moved to an ice bath, and $8 \mathrm{~mL}$ TABF (1.0 $M$ in THF) solution was slowly addition to reaction mixture. The reaction solution was stirred at $0{ }^{\circ} \mathrm{C}$ until consumption of the reactants (monitored by TLC) was complete (about $5 \mathrm{~min}$ ). Then the reaction solution was purified by column chromatography on silica gel without any additional treatment (hexanes : EtOAc $=10: 1)$ to give 4aa $(904 \mathrm{mg})$ as pure products in $75 \%$ yield with $99 \%$ ee. 
4. NMR Spectra of New compounds

${ }^{1} \mathrm{H}-\mathrm{NMR}\left(500 \mathrm{MHz}, \mathrm{CDCl}_{3}\right.$ ) of compound 4aa
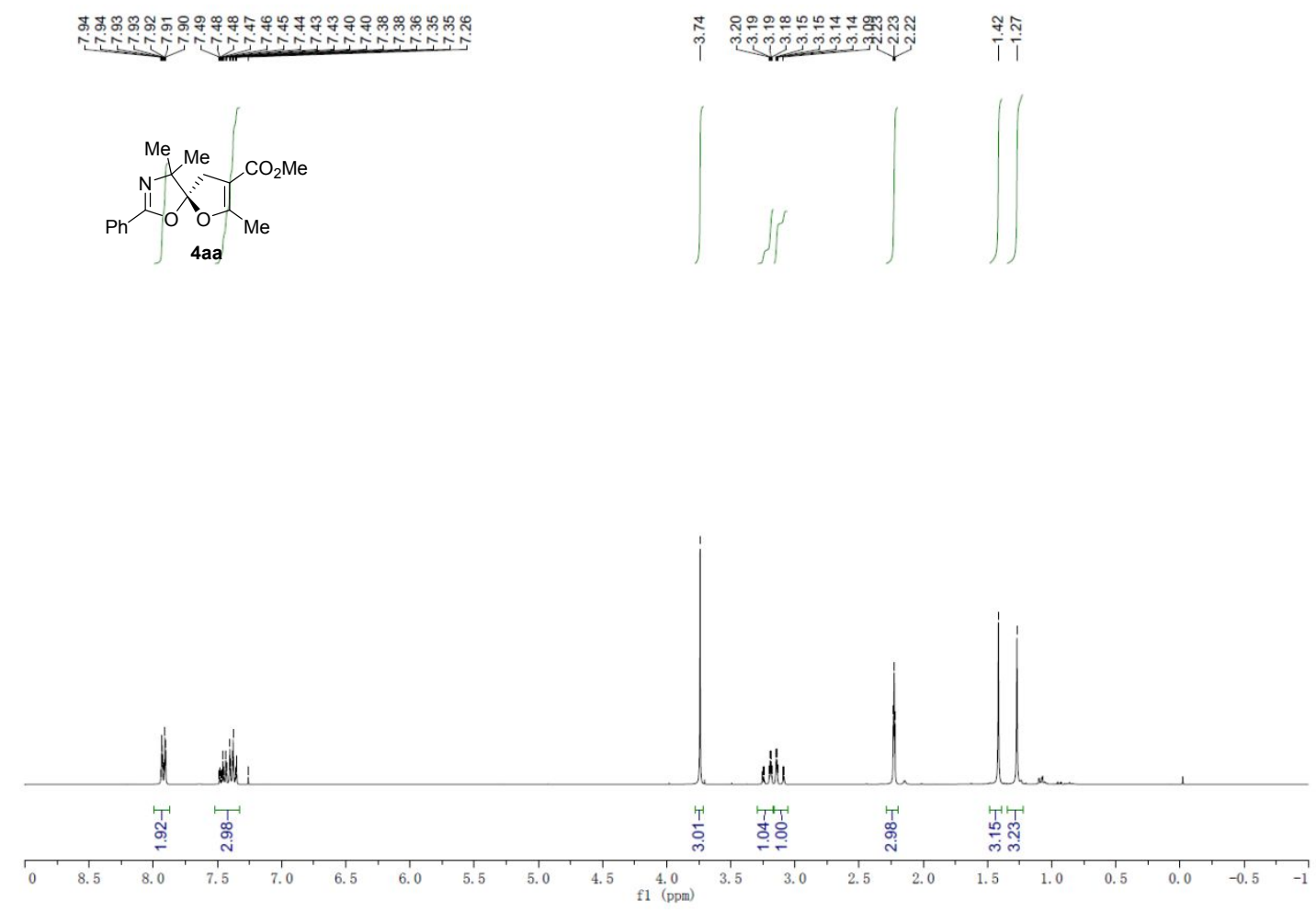

${ }^{13} \mathrm{C}-\mathrm{NMR}\left(125 \mathrm{MHz}, \mathrm{CDCl}_{3}\right)$ of compound 4aa

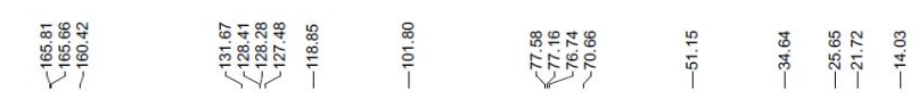

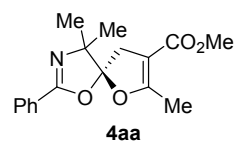

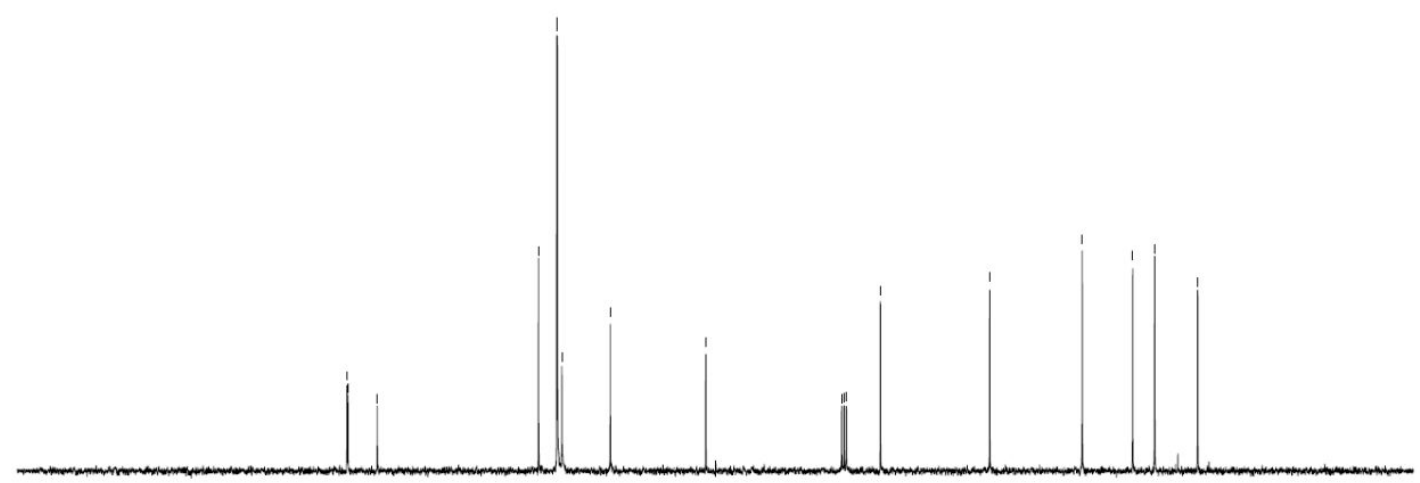

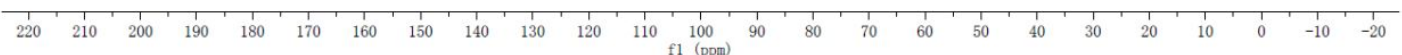


${ }^{1}$ H-NMR (500 MHz, $\mathrm{CDCl}_{3}$ ) of compound 4ab

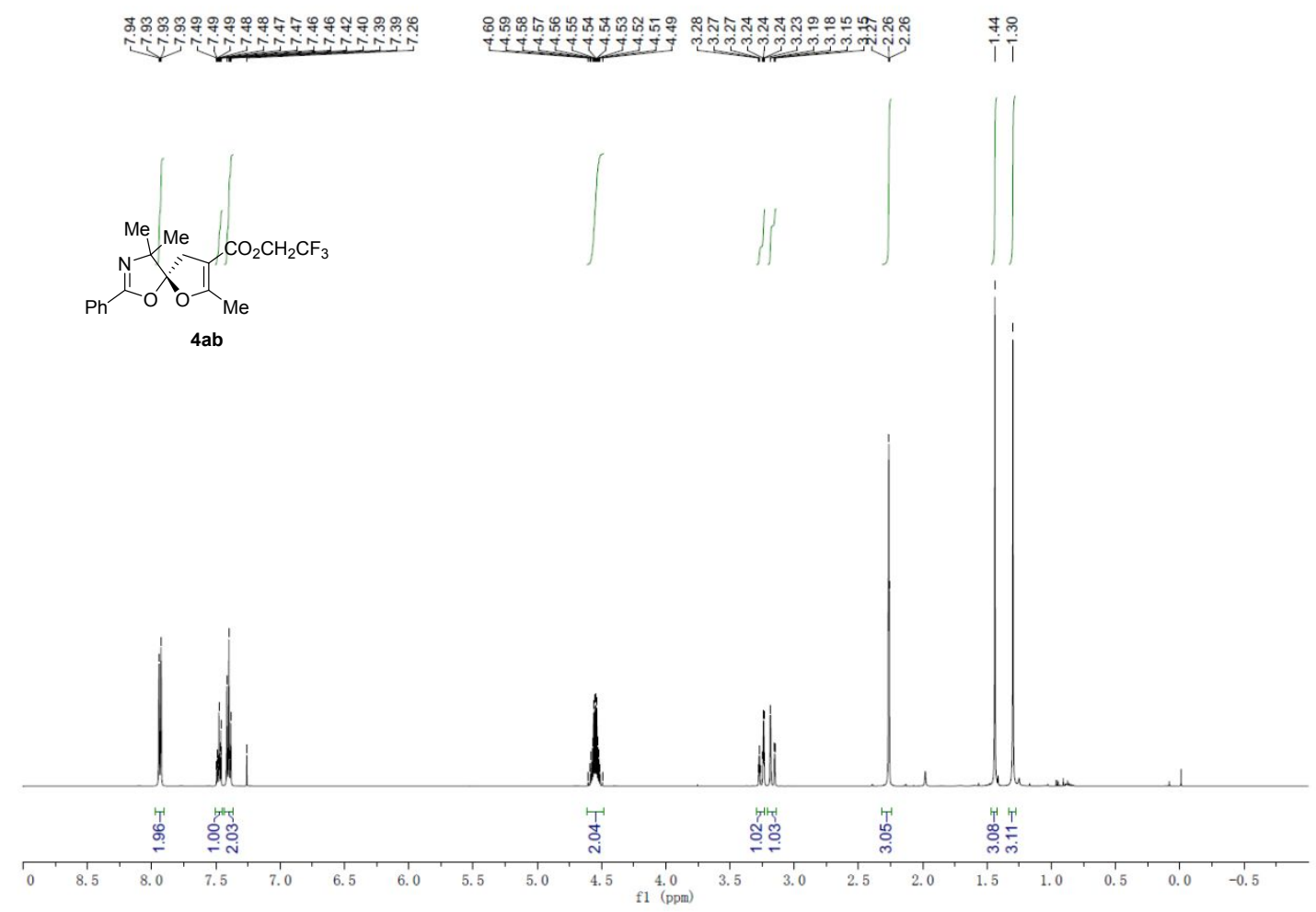

${ }^{13} \mathrm{C}-\mathrm{NMR}\left(125 \mathrm{MHz}, \mathrm{CDCl}_{3}\right)$ of compound 4ab

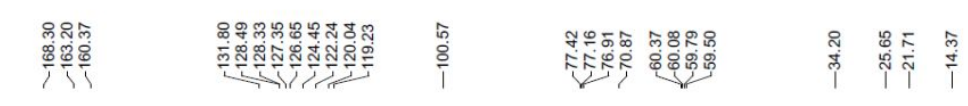
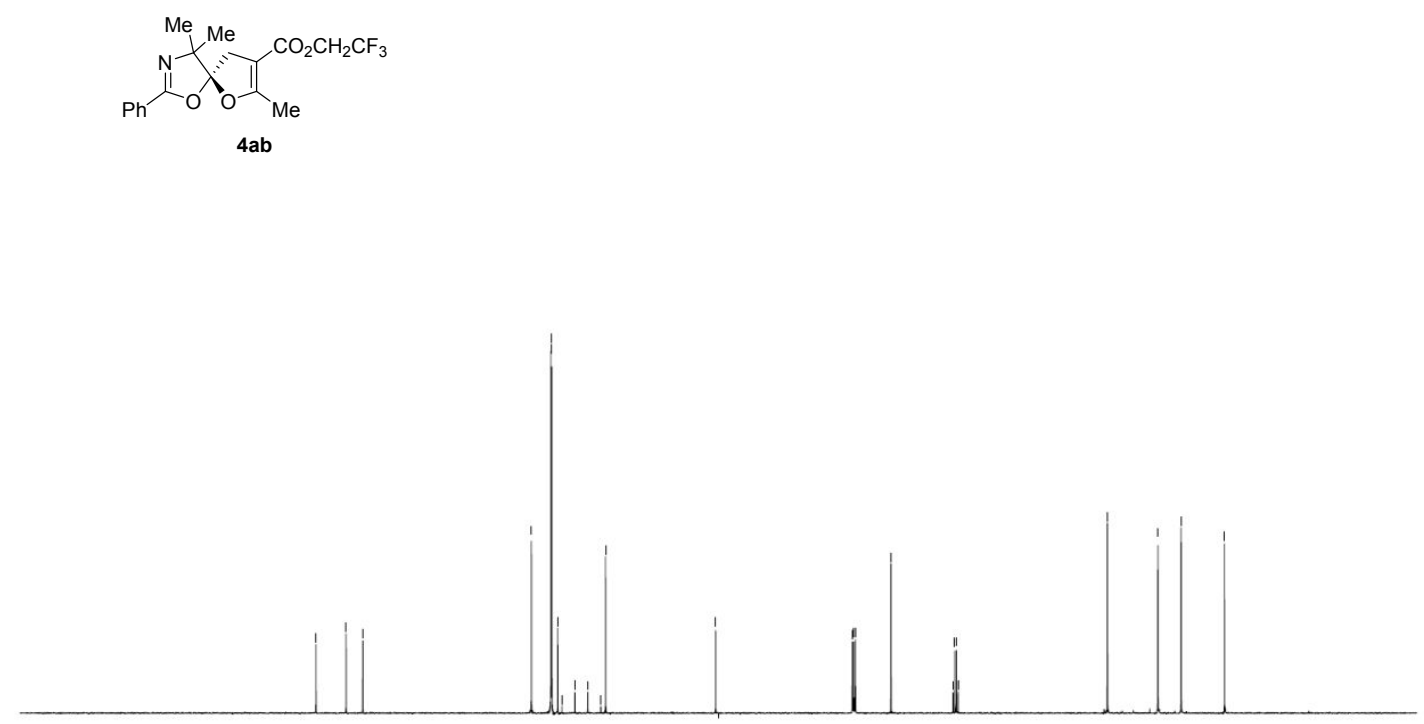

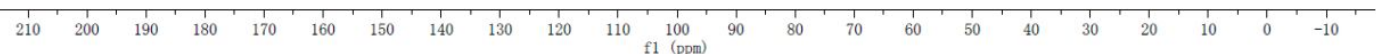


${ }^{1} \mathrm{H}-\mathrm{NMR}$ (500 MHz, $\mathrm{CDCl}_{3}$ ) of compound $4 \mathrm{ac}$
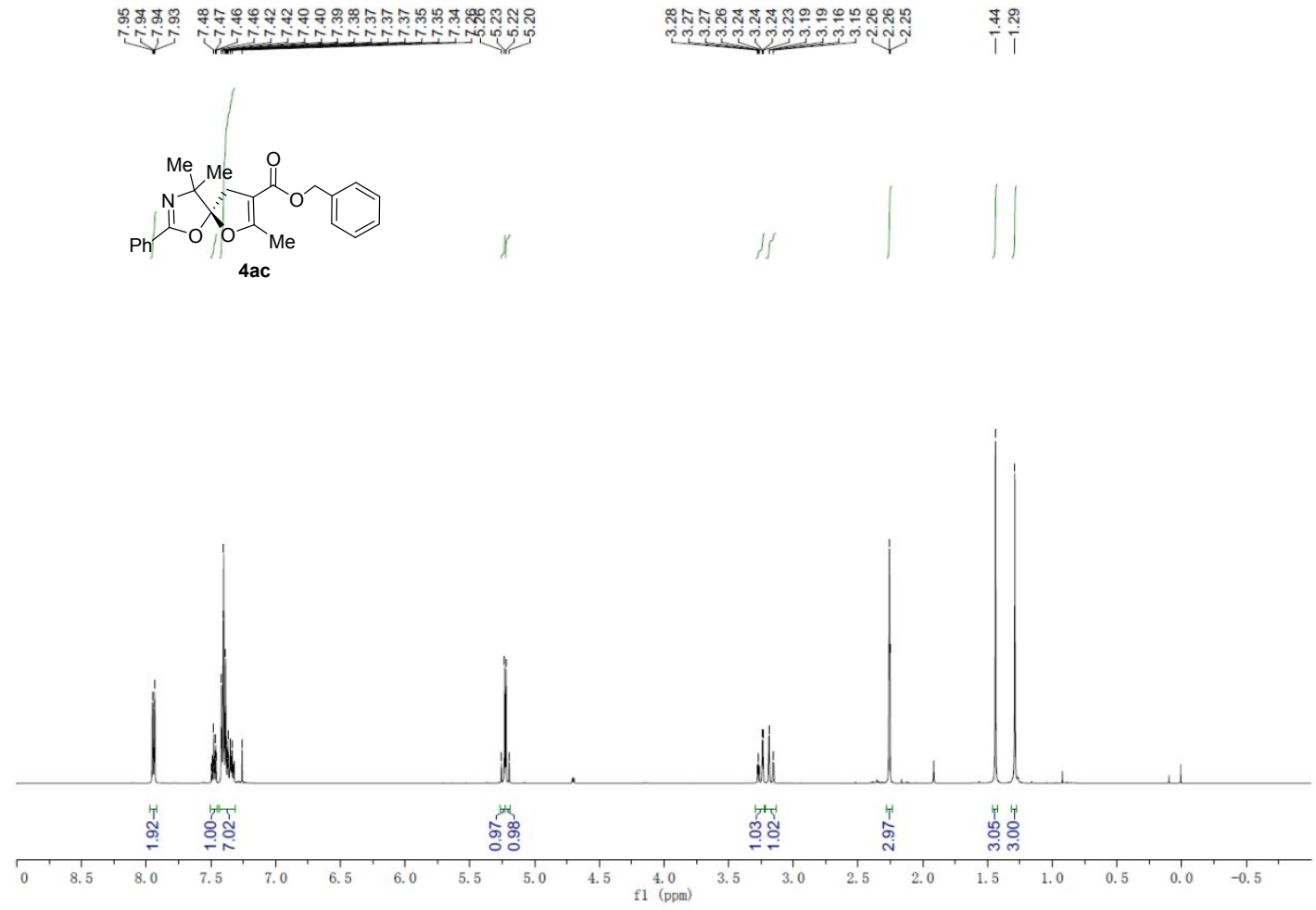

${ }^{13} \mathrm{C}-\mathrm{NMR}\left(125 \mathrm{MHz}, \mathrm{CDCl}_{3}\right)$ of compound $4 \mathrm{ac}$
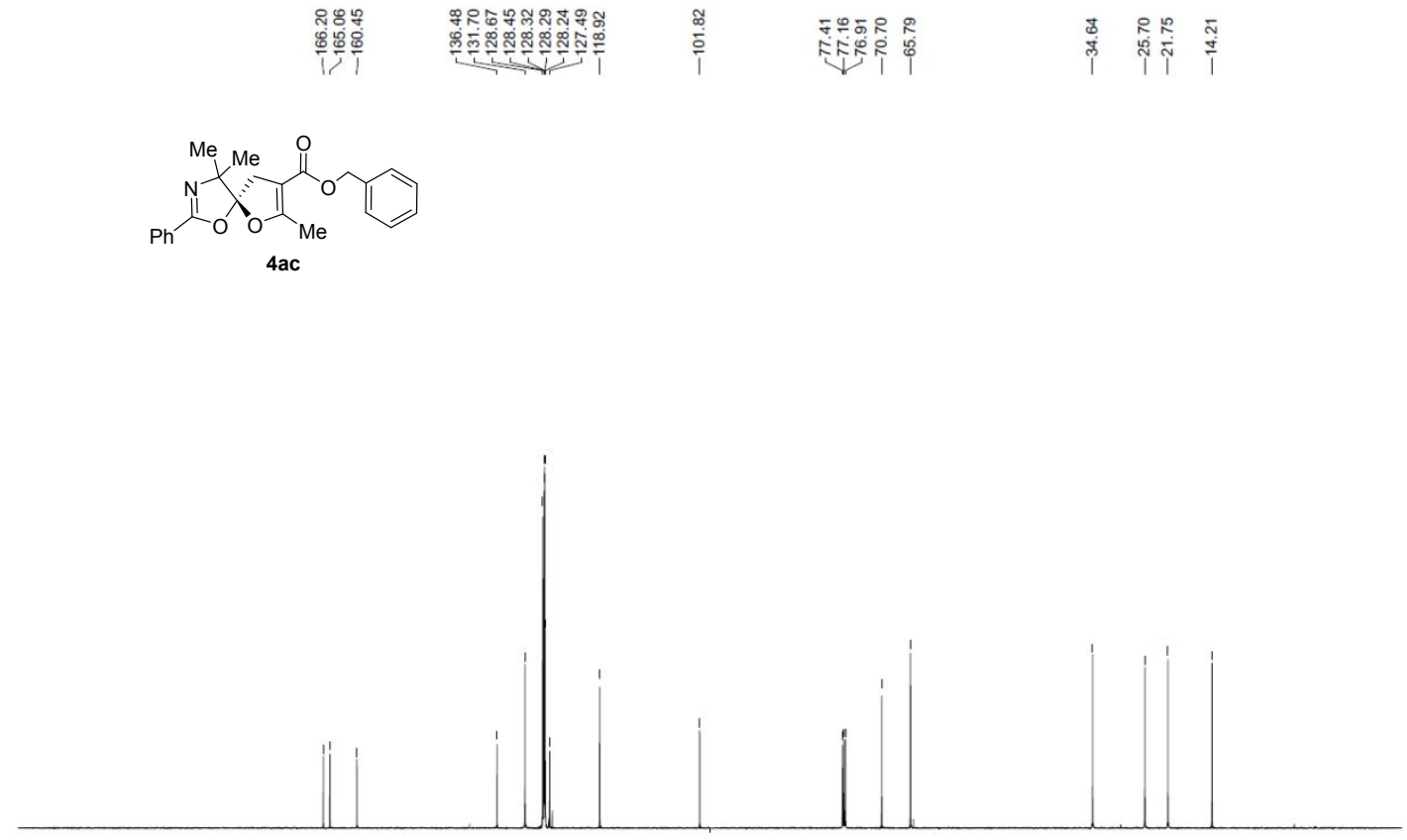
${ }^{1} \mathrm{H}-\mathrm{NMR}\left(500 \mathrm{MHz}, \mathrm{CDCl}_{3}\right)$ of compound 4ad
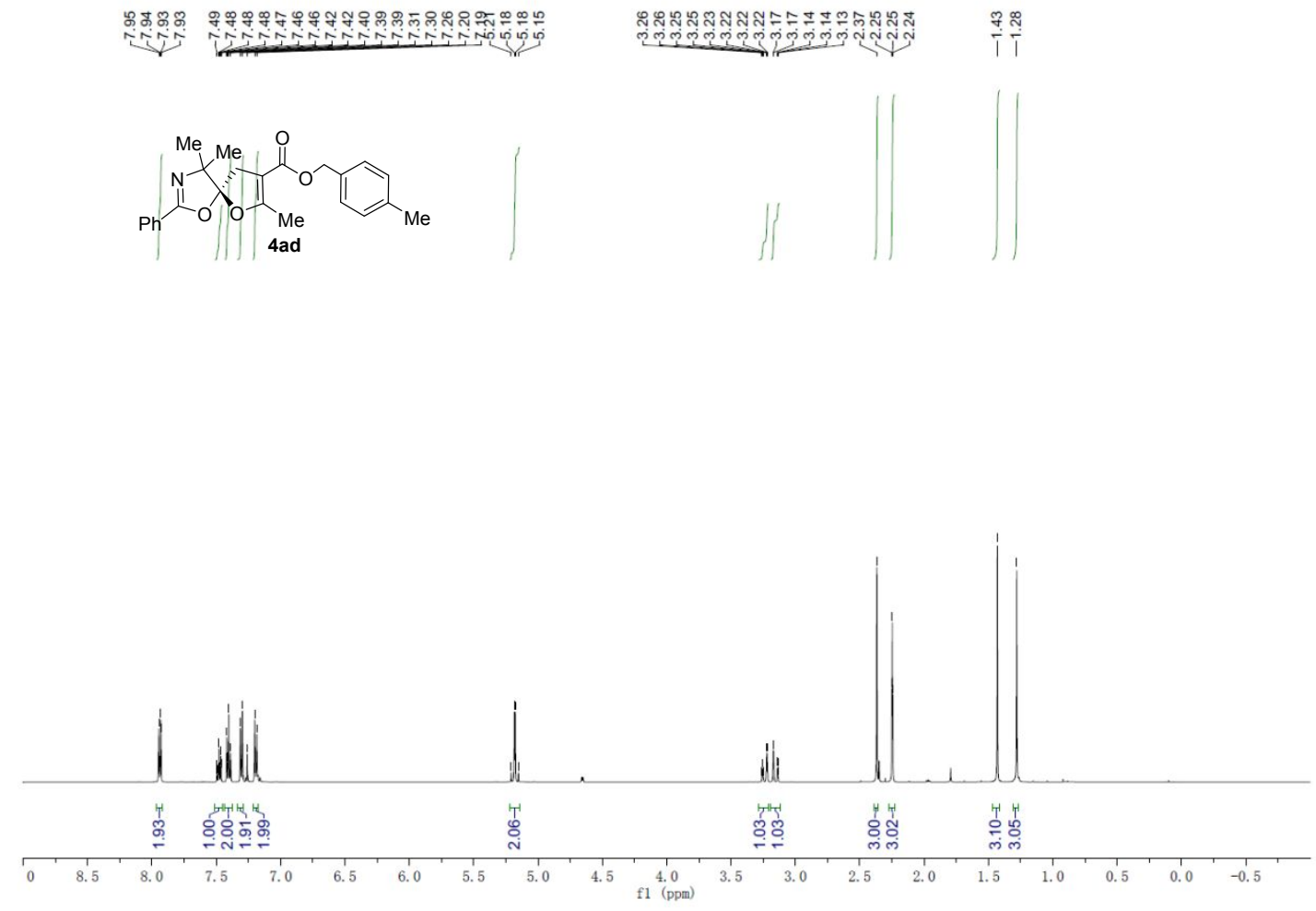

${ }^{13} \mathrm{C}-\mathrm{NMR}\left(125 \mathrm{MHz}, \mathrm{CDCl}_{3}\right)$ of compound 4ad

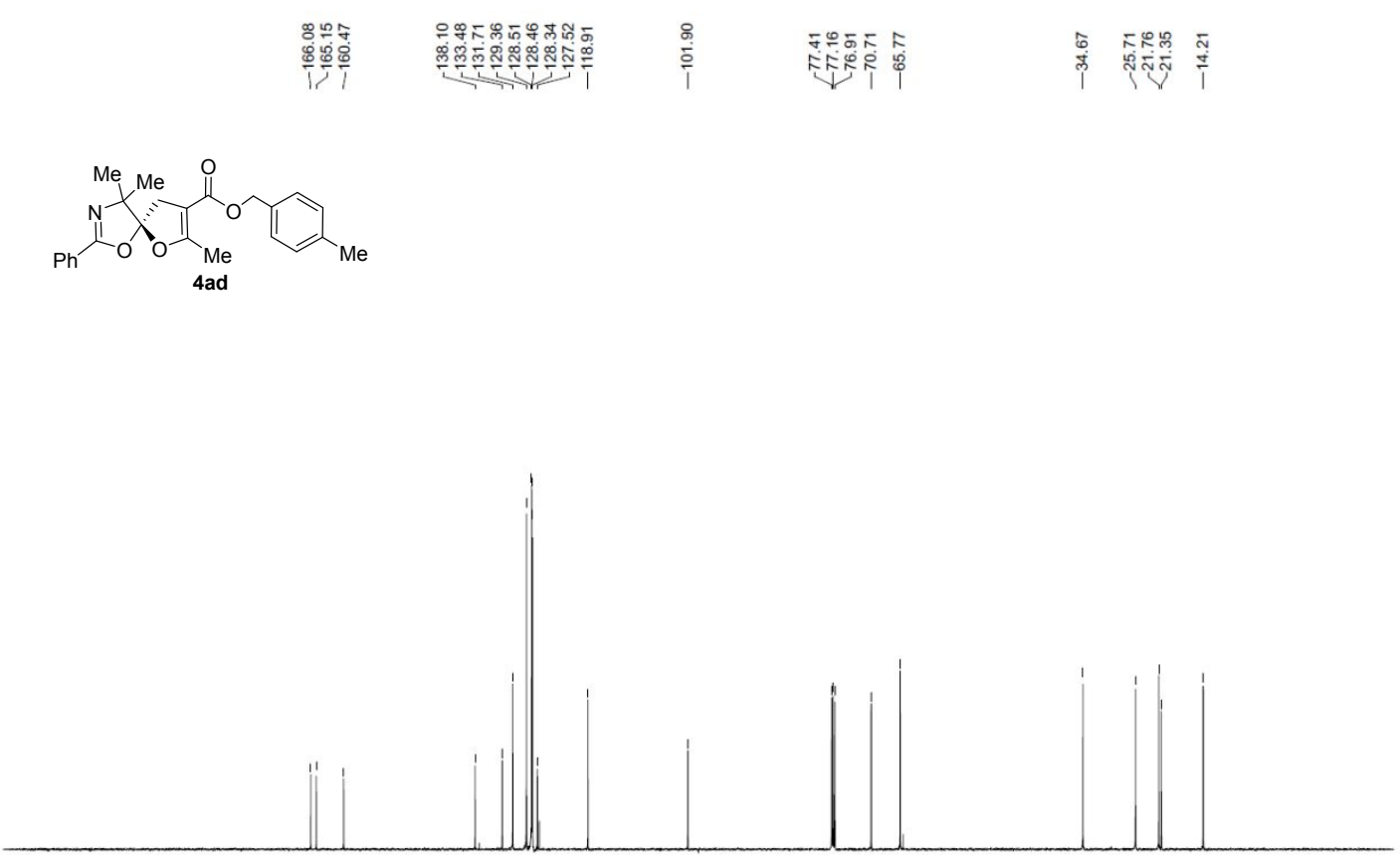

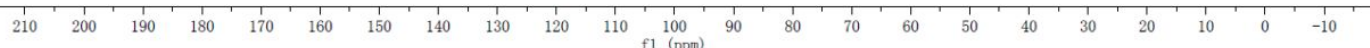


${ }^{1} \mathrm{H}-\mathrm{NMR}$ (500 MHz, $\mathrm{CDCl}_{3}$ ) of compound 4ae
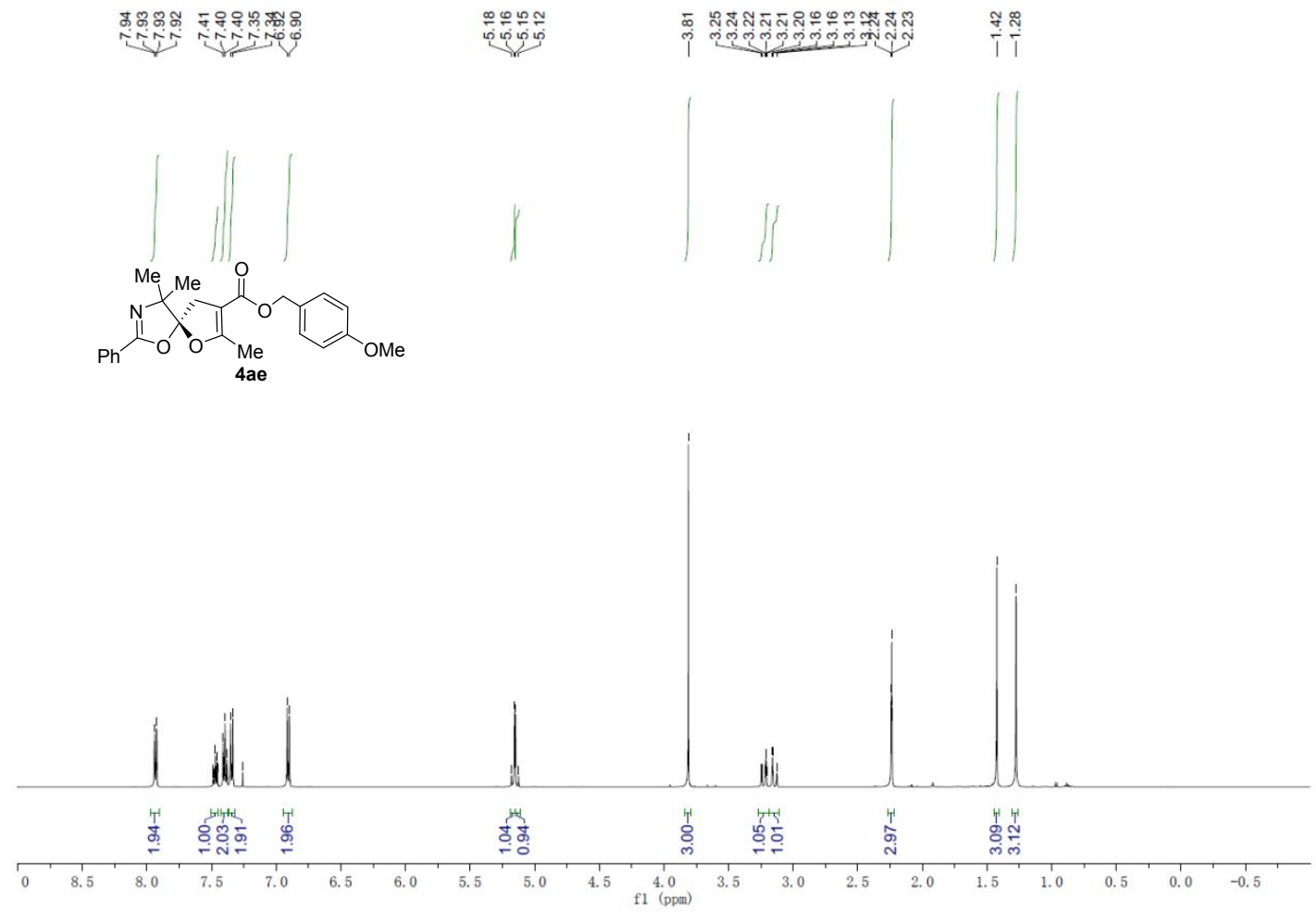

${ }^{13} \mathrm{C}-\mathrm{NMR}$ (125 MHz, $\mathrm{CDCl}_{3}$ ) of compound 4ae

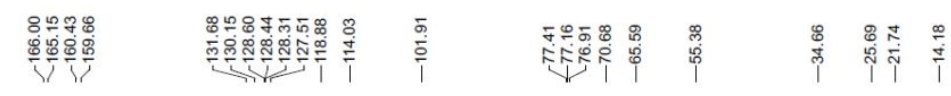
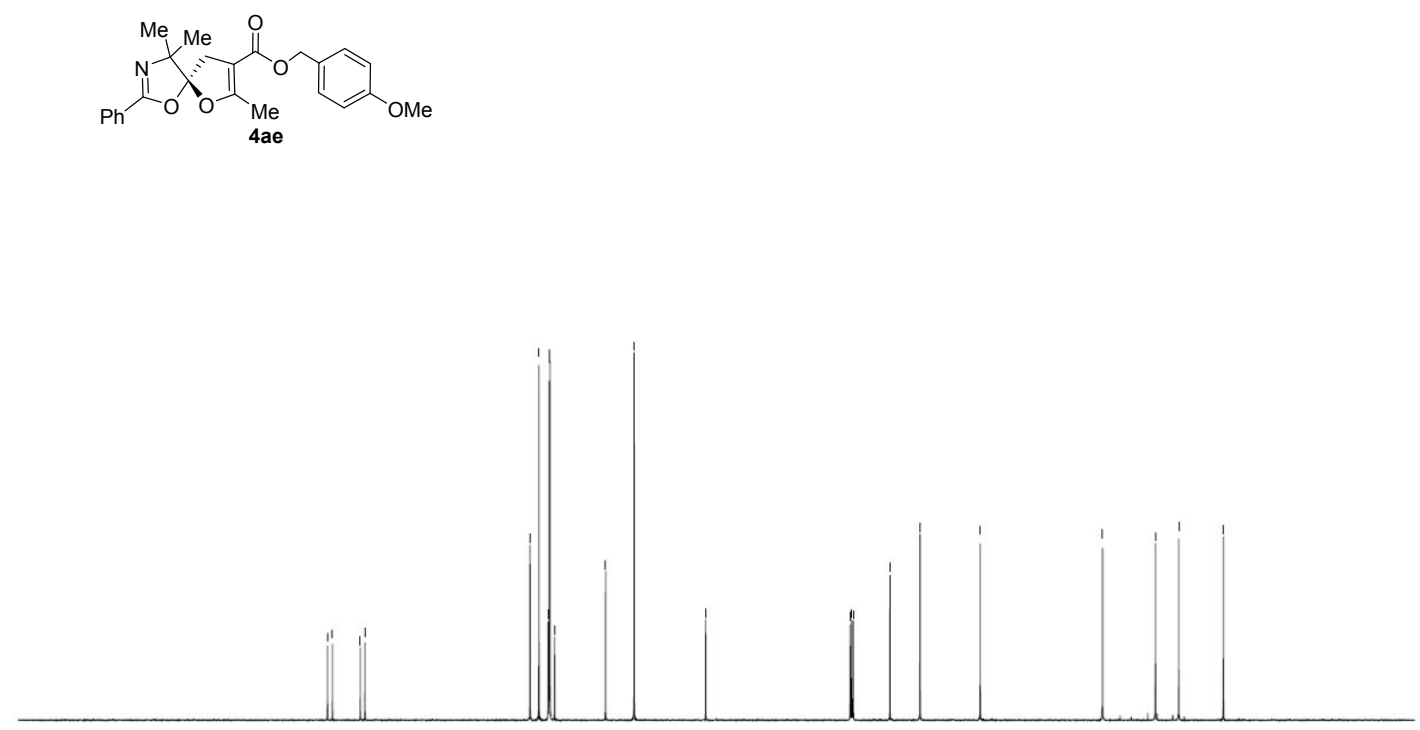

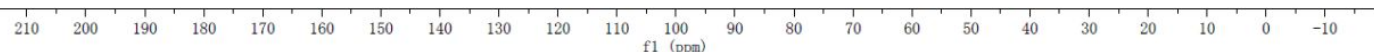


${ }^{1} \mathrm{H}-\mathrm{NMR}\left(500 \mathrm{MHz}, \mathrm{CDCl}_{3}\right.$ ) of compound 4af

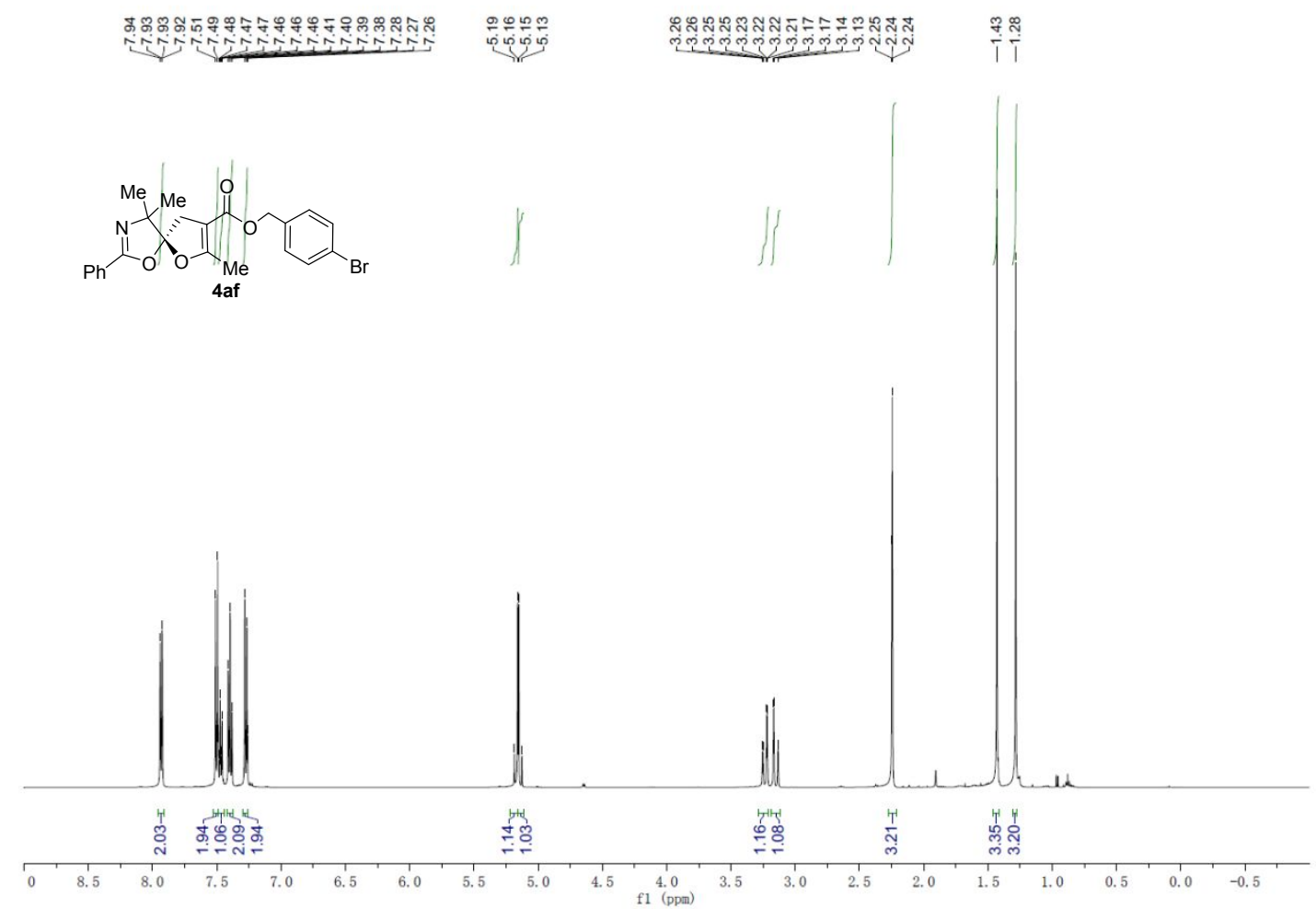

${ }^{13} \mathrm{C}$-NMR (125 MHz, $\left.\mathrm{CDCl}_{3}\right)$ of compound 4af
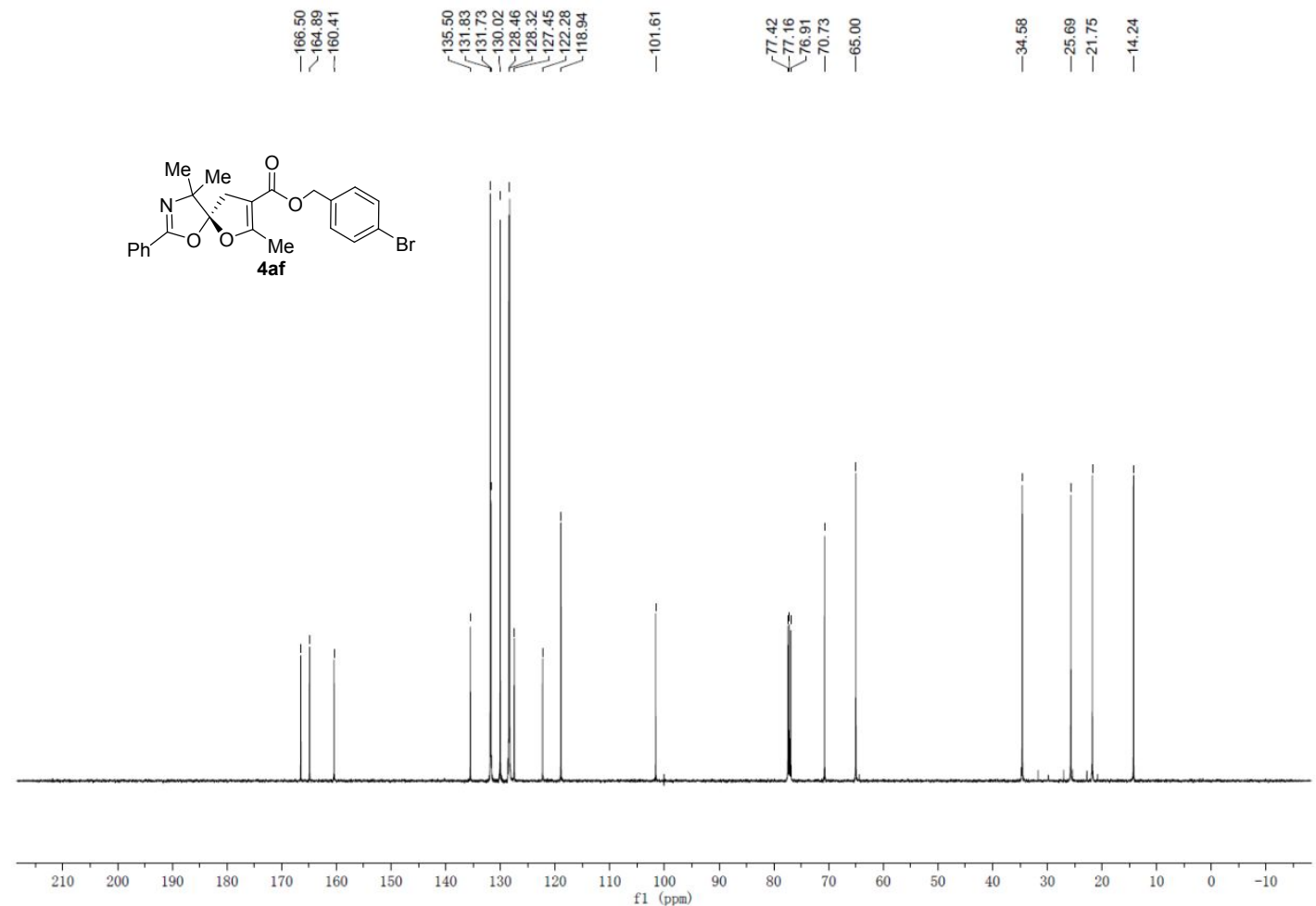
${ }^{1} \mathrm{H}-\mathrm{NMR}\left(500 \mathrm{MHz}, \mathrm{CDCl}_{3}\right)$ of compound $4 \mathrm{ag}$
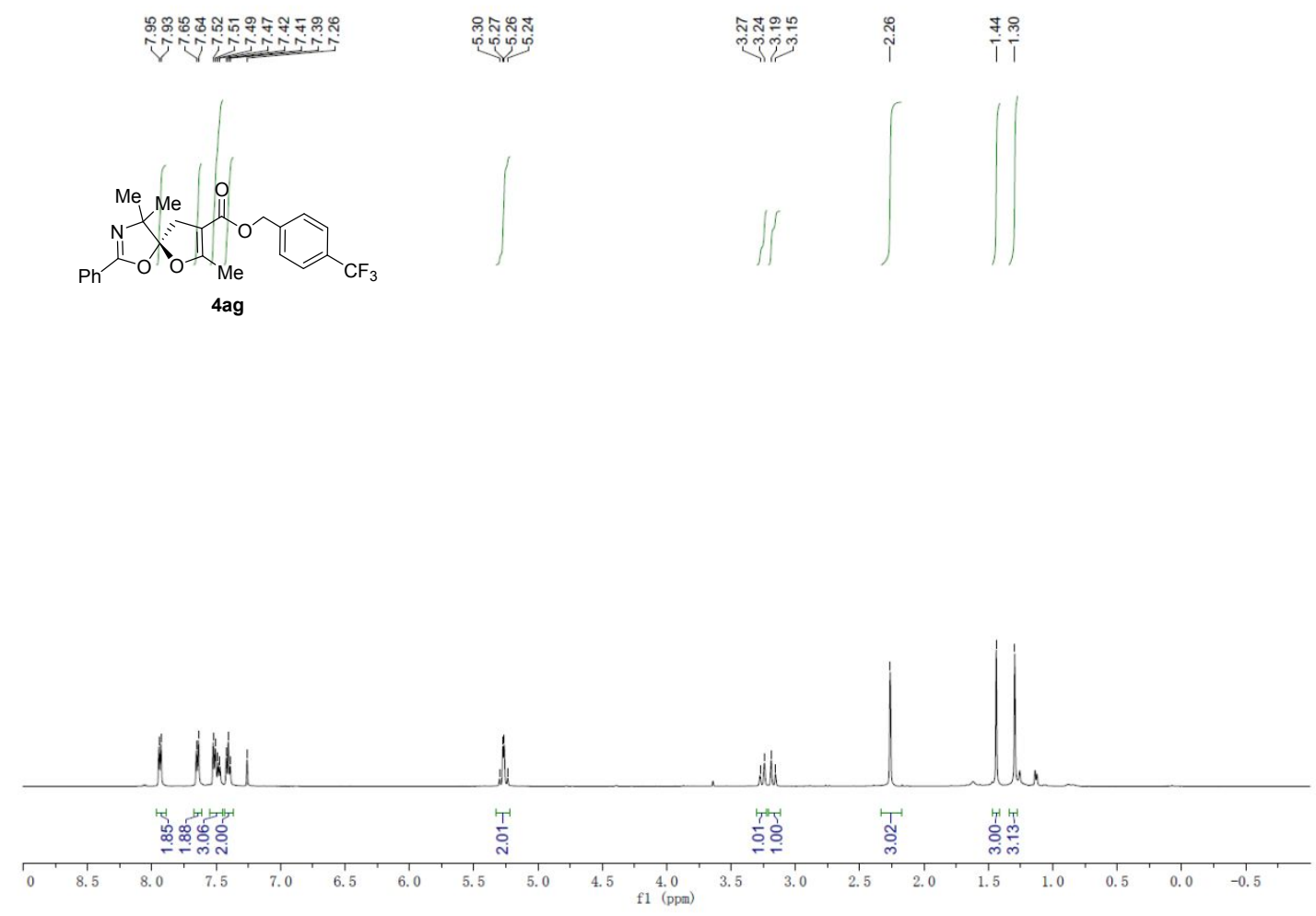

${ }^{13} \mathrm{C}-\mathrm{NMR}\left(125 \mathrm{MHz}, \mathrm{CDCl}_{3}\right)$ of compound 4ag

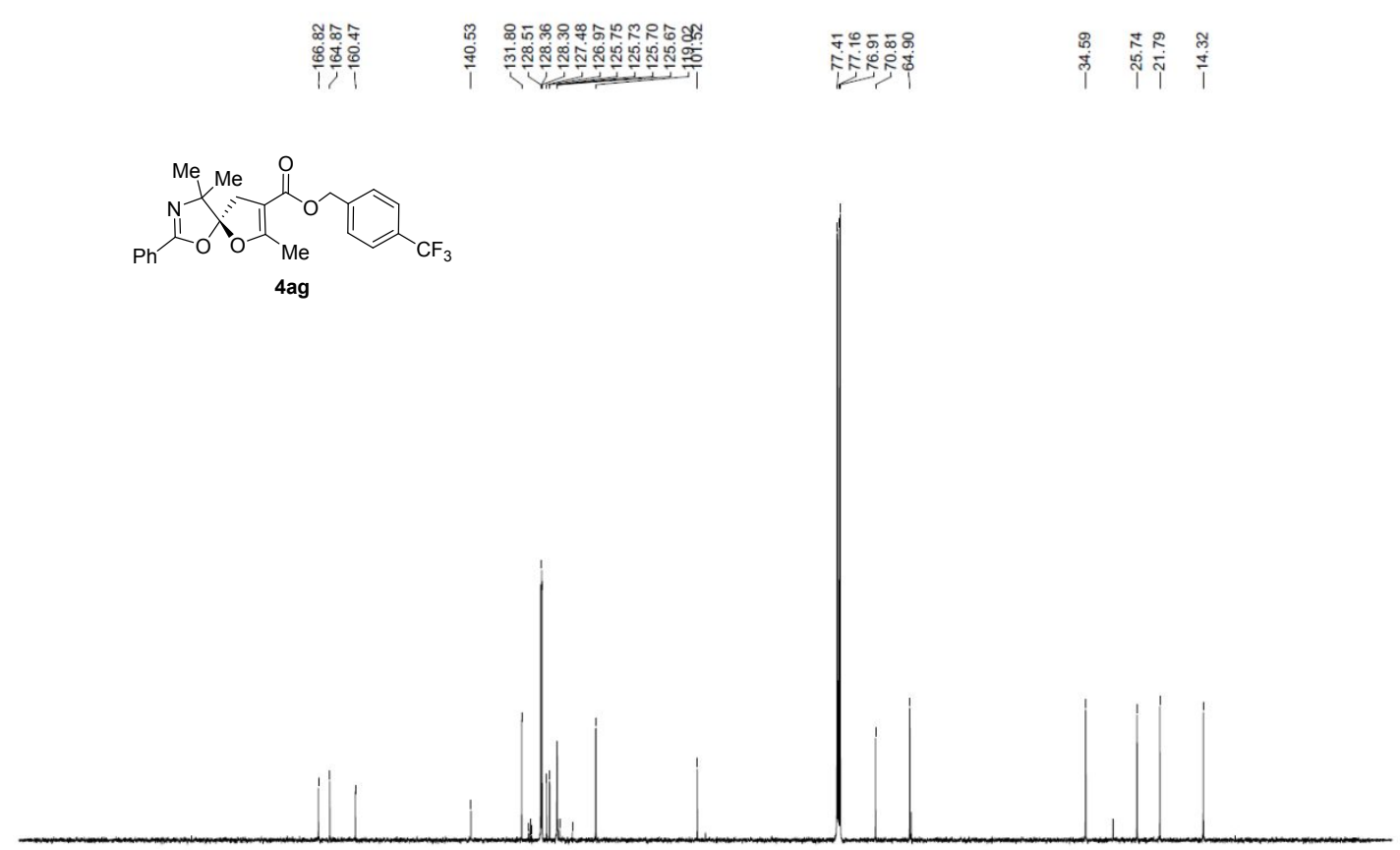


${ }^{1} \mathrm{H}-\mathrm{NMR}\left(500 \mathrm{MHz}, \mathrm{CDCl}_{3}\right)$ of compound $4 \mathrm{ah}$

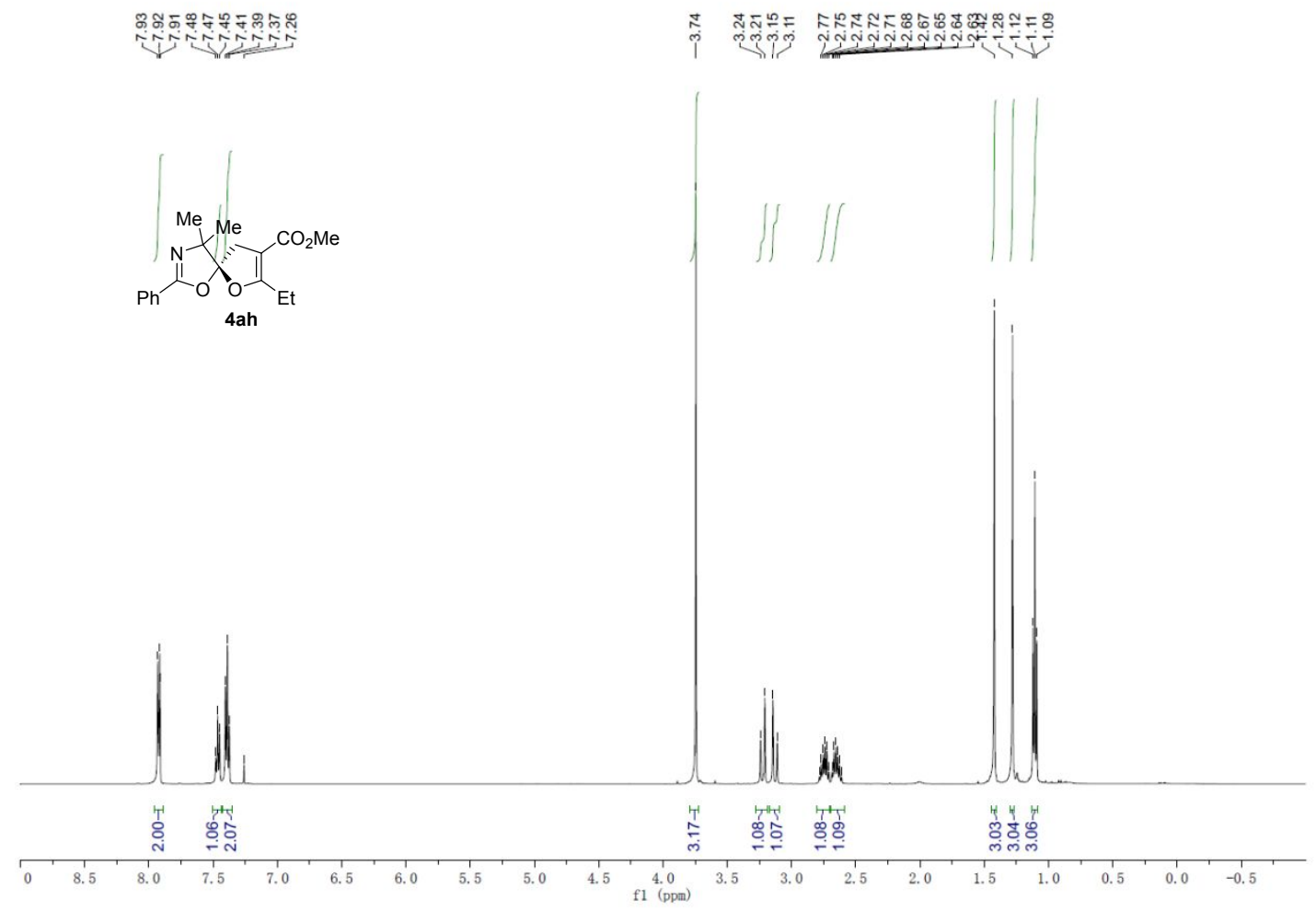

${ }^{13} \mathrm{C}$-NMR (125 MHz, $\left.\mathrm{CDCl}_{3}\right)$ of compound 4ah

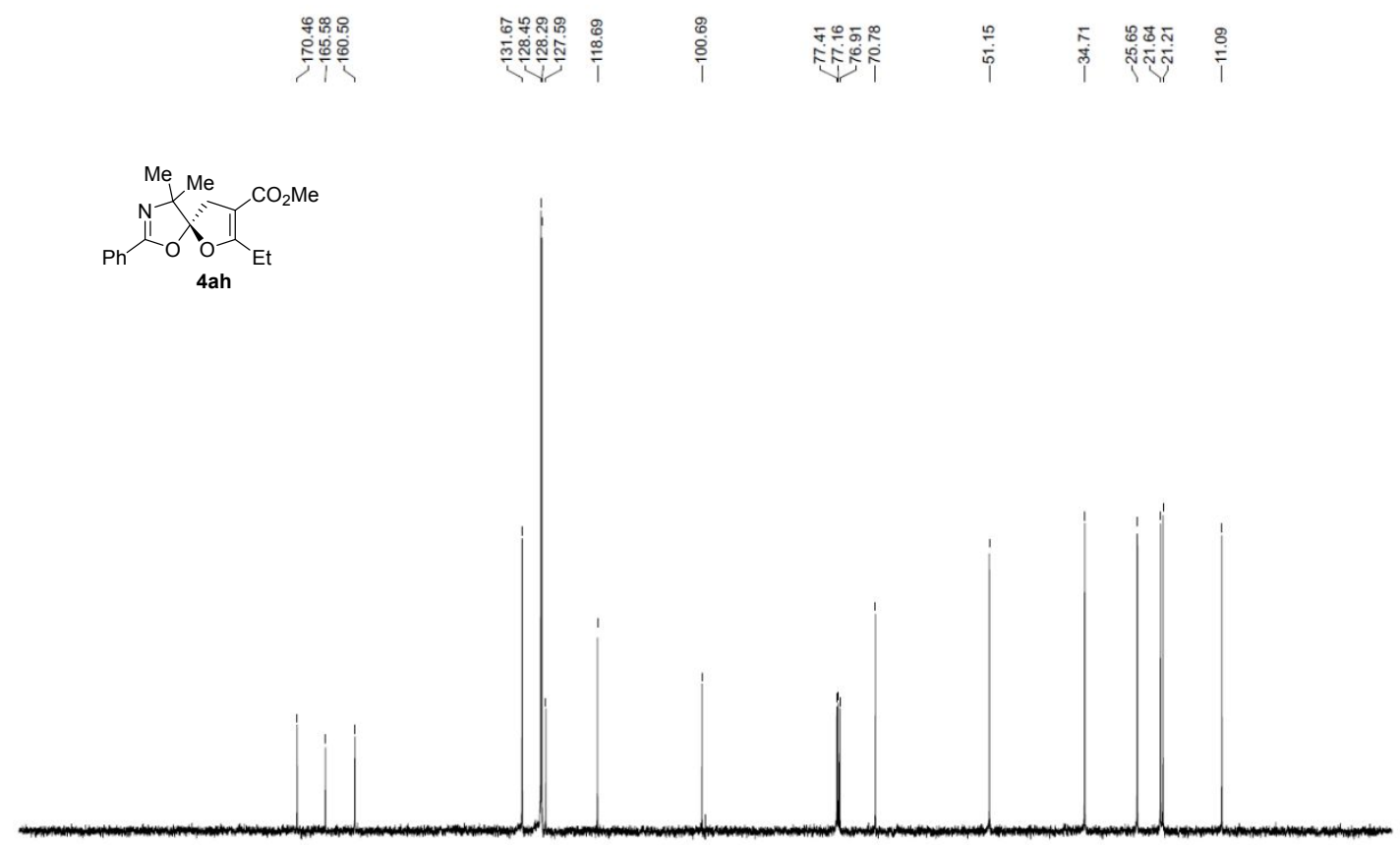

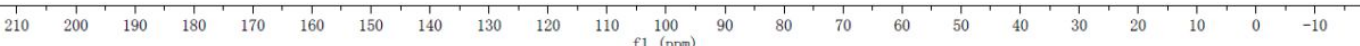


${ }^{1} \mathrm{H}-\mathrm{NMR}\left(500 \mathrm{MHz}, \mathrm{CDCl}_{3}\right)$ of compound 4ai

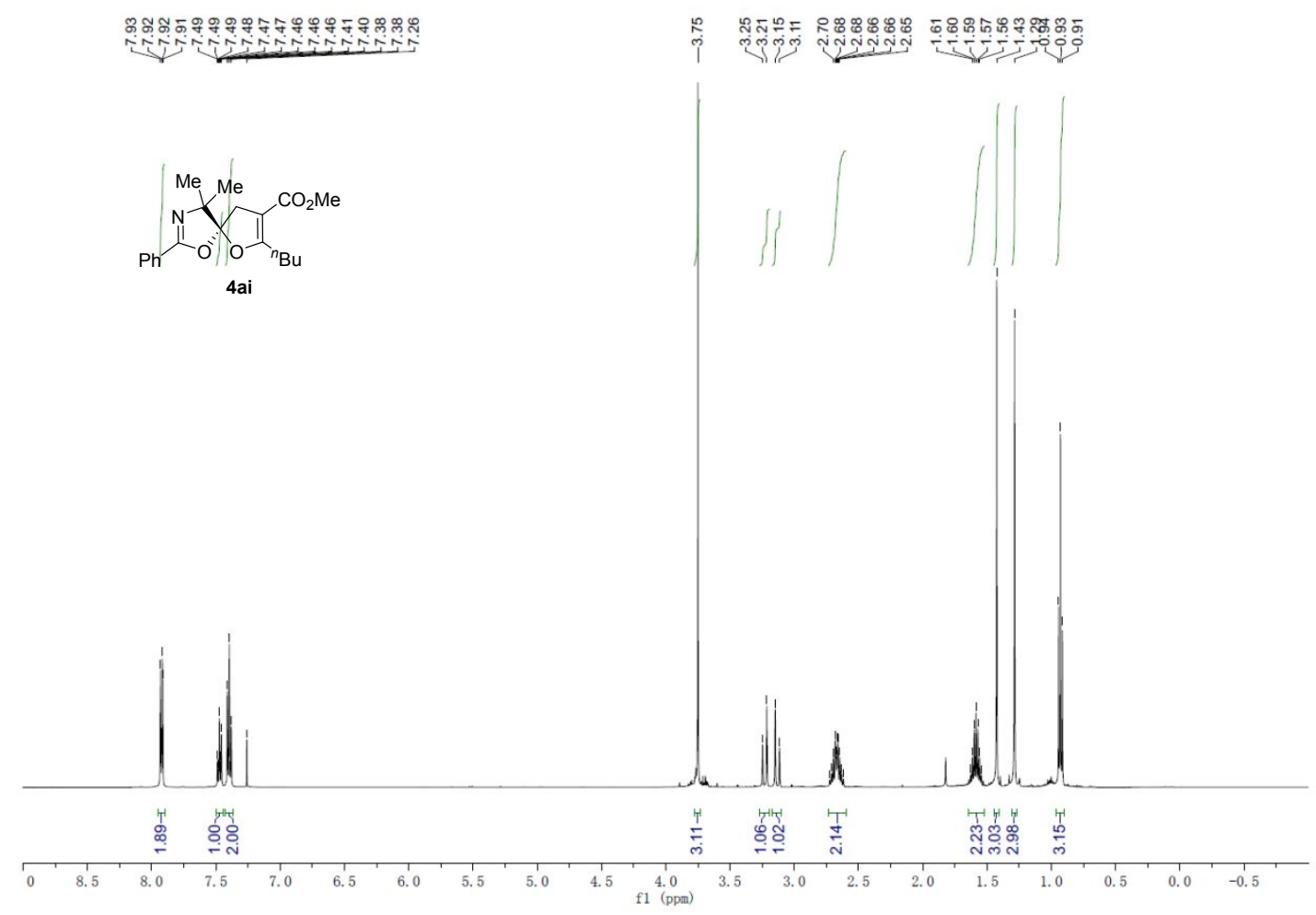

${ }^{13} \mathrm{C}$-NMR (125 MHz, $\left.\mathrm{CDCl}_{3}\right)$ of compound 4ai

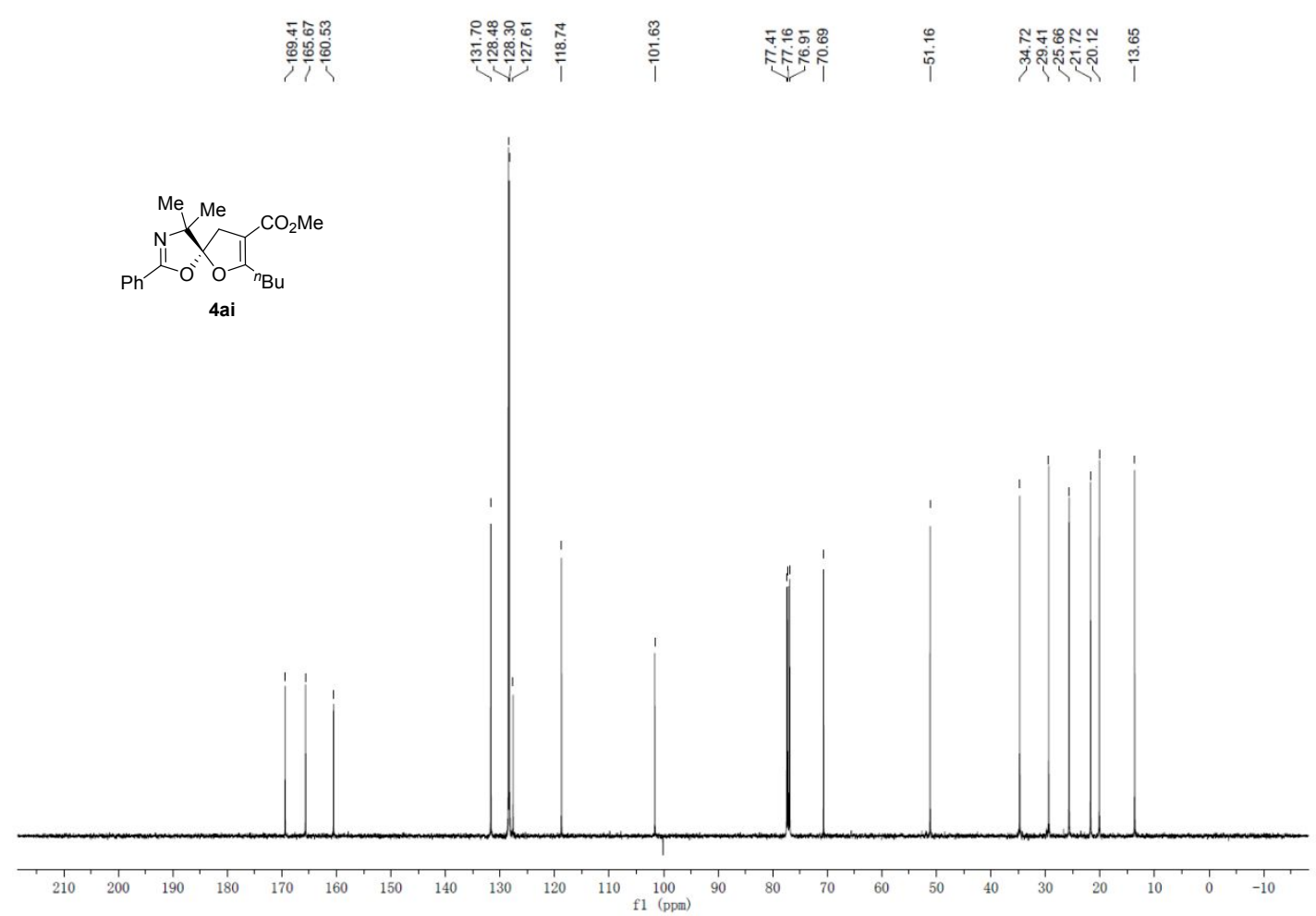


${ }^{1} \mathrm{H}-\mathrm{NMR}\left(500 \mathrm{MHz}, \mathrm{CDCl}_{3}\right)$ of compound 4aj

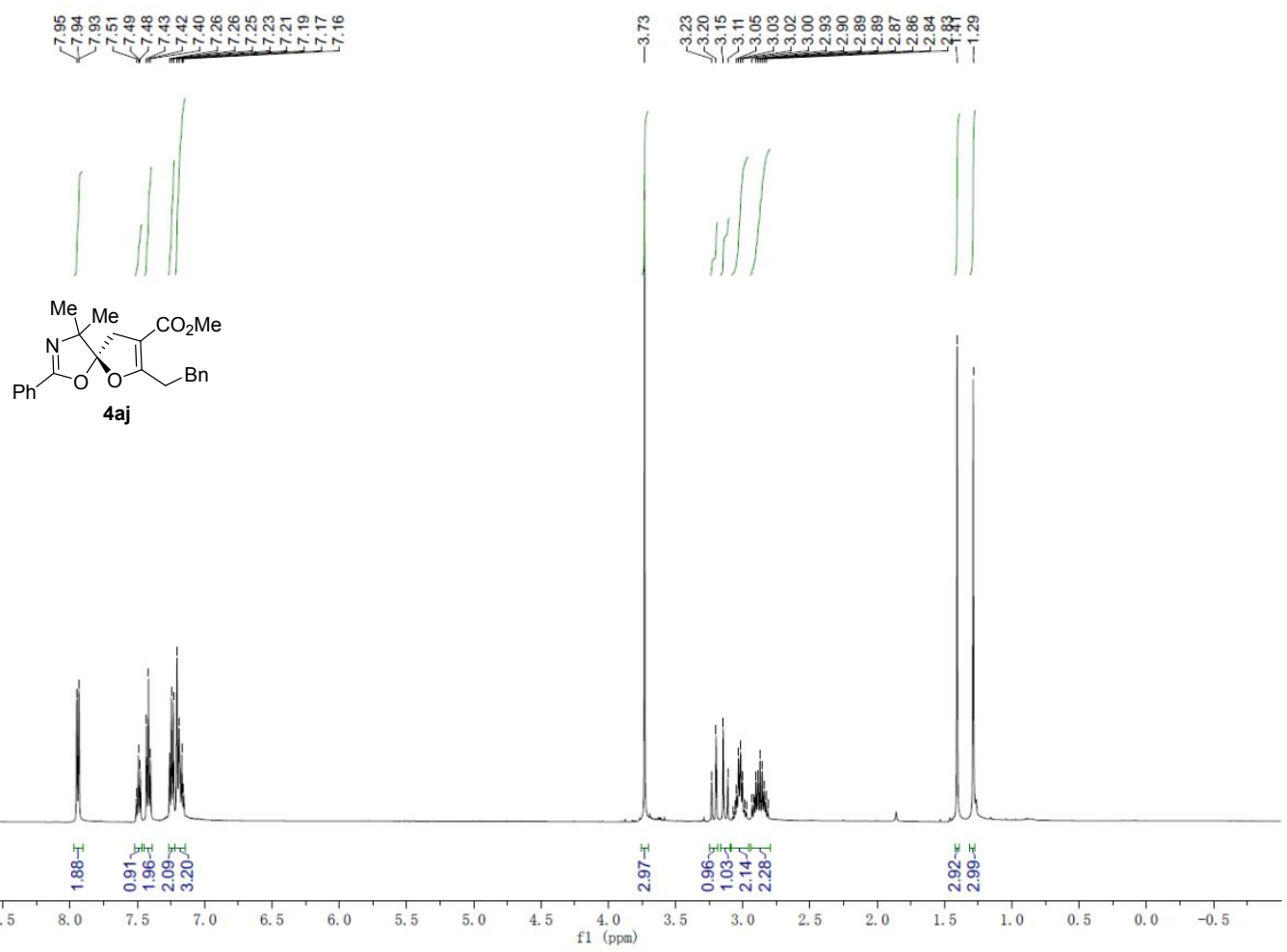

${ }^{13} \mathrm{C}-\mathrm{NMR}\left(125 \mathrm{MHz}, \mathrm{CDCl}_{3}\right)$ of compound 4aj

\begin{tabular}{|c|c|}
\hline 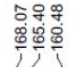 & 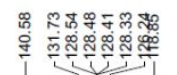 \\
\hline
\end{tabular}

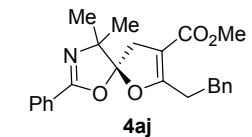

4aj

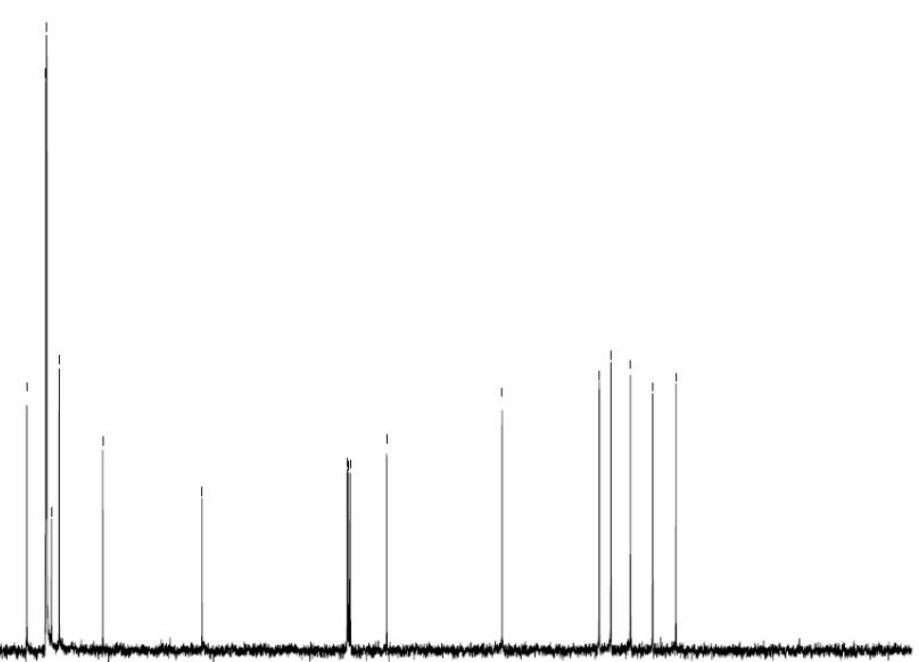

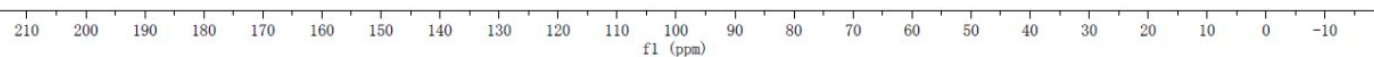


${ }^{1} \mathrm{H}-\mathrm{NMR}\left(500 \mathrm{MHz}, \mathrm{CDCl}_{3}\right)$ of compound $4 \mathrm{ba}$

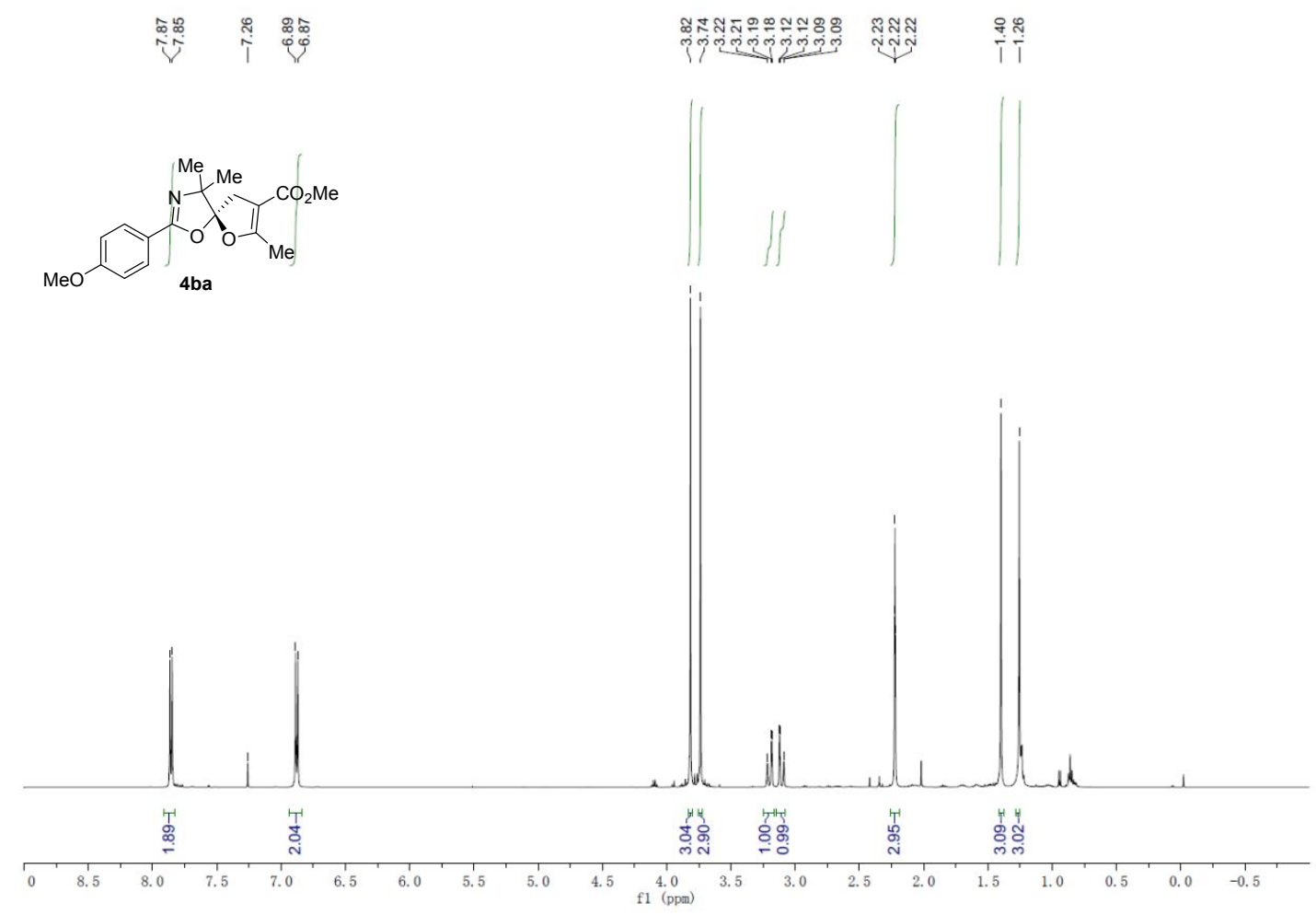

${ }^{13} \mathrm{C}-\mathrm{NMR}\left(125 \mathrm{MHz}, \mathrm{CDCl}_{3}\right)$ of compound $4 \mathrm{ba}$

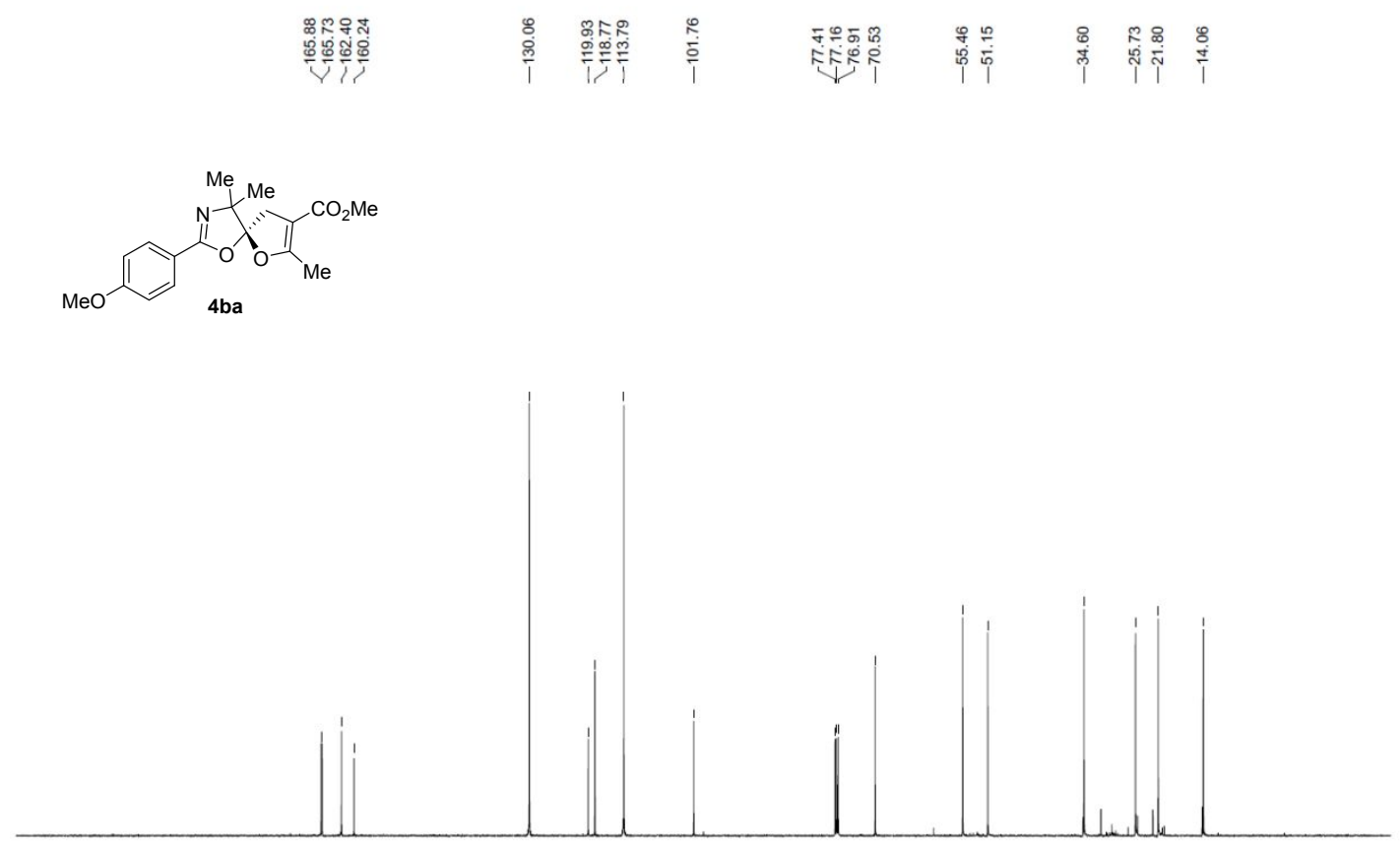

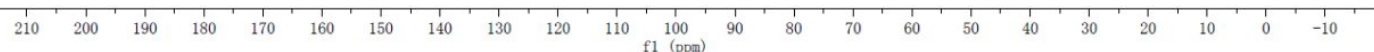


${ }^{1} \mathrm{H}-\mathrm{NMR}\left(500 \mathrm{MHz}, \mathrm{CDCl}_{3}\right)$ of compound $4 \mathrm{ca}$

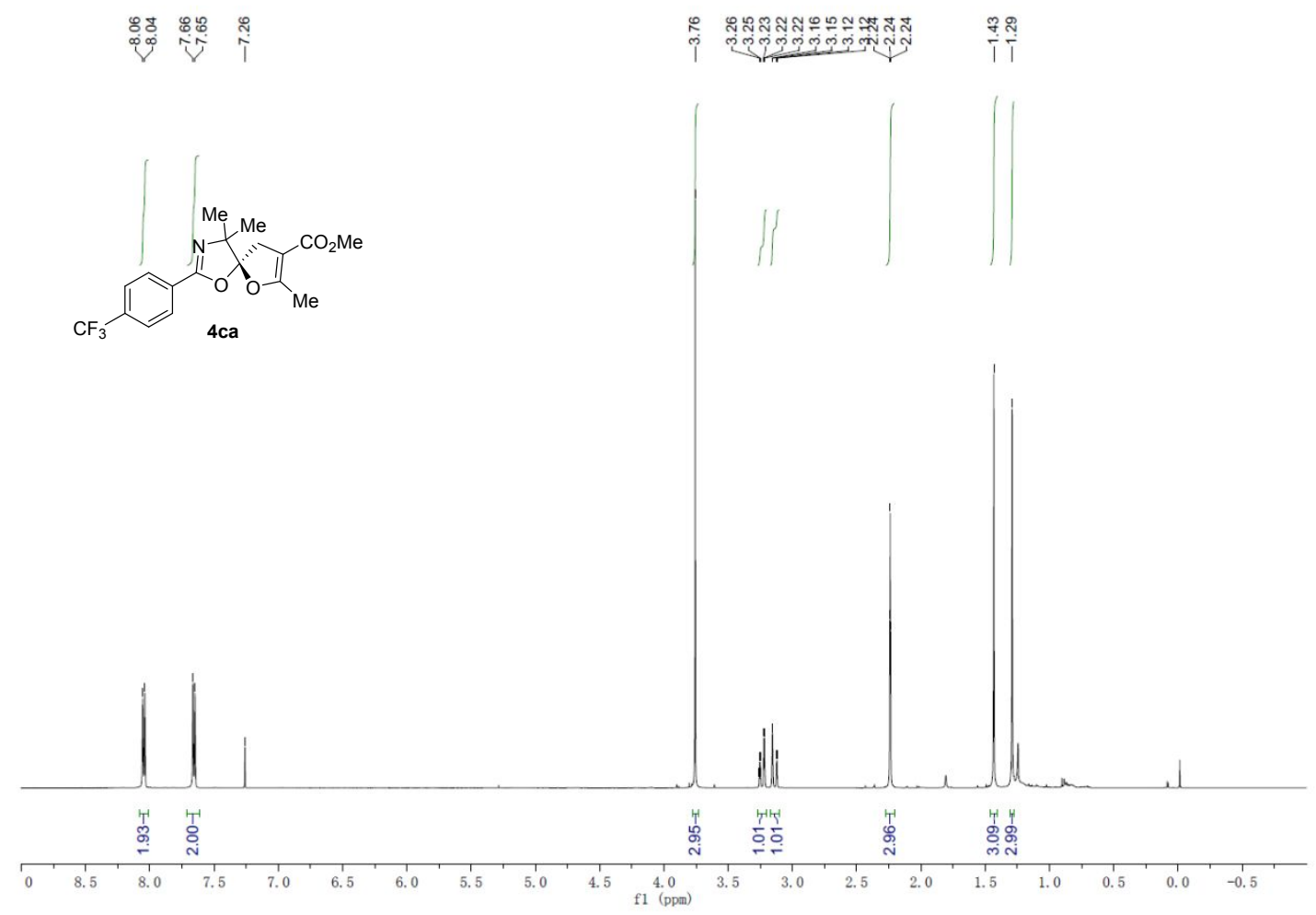

${ }^{13} \mathrm{C}-\mathrm{NMR}\left(125 \mathrm{MHz}, \mathrm{CDCl}_{3}\right)$ of compound 4ca
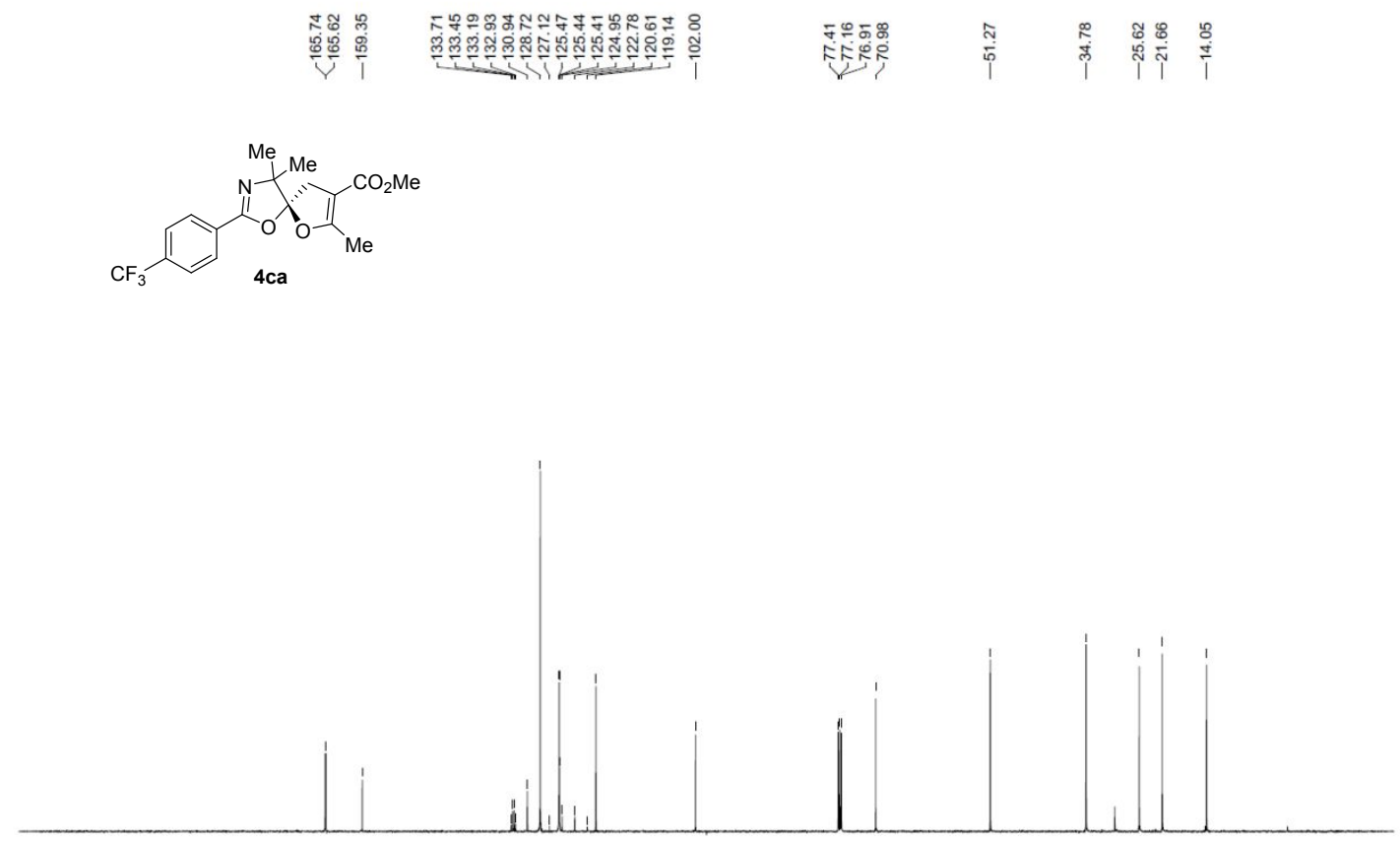

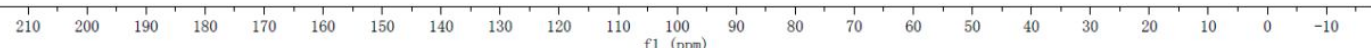


${ }^{1} \mathrm{H}-\mathrm{NMR}\left(500 \mathrm{MHz}, \mathrm{CDCl}_{3}\right)$ of compound 4da

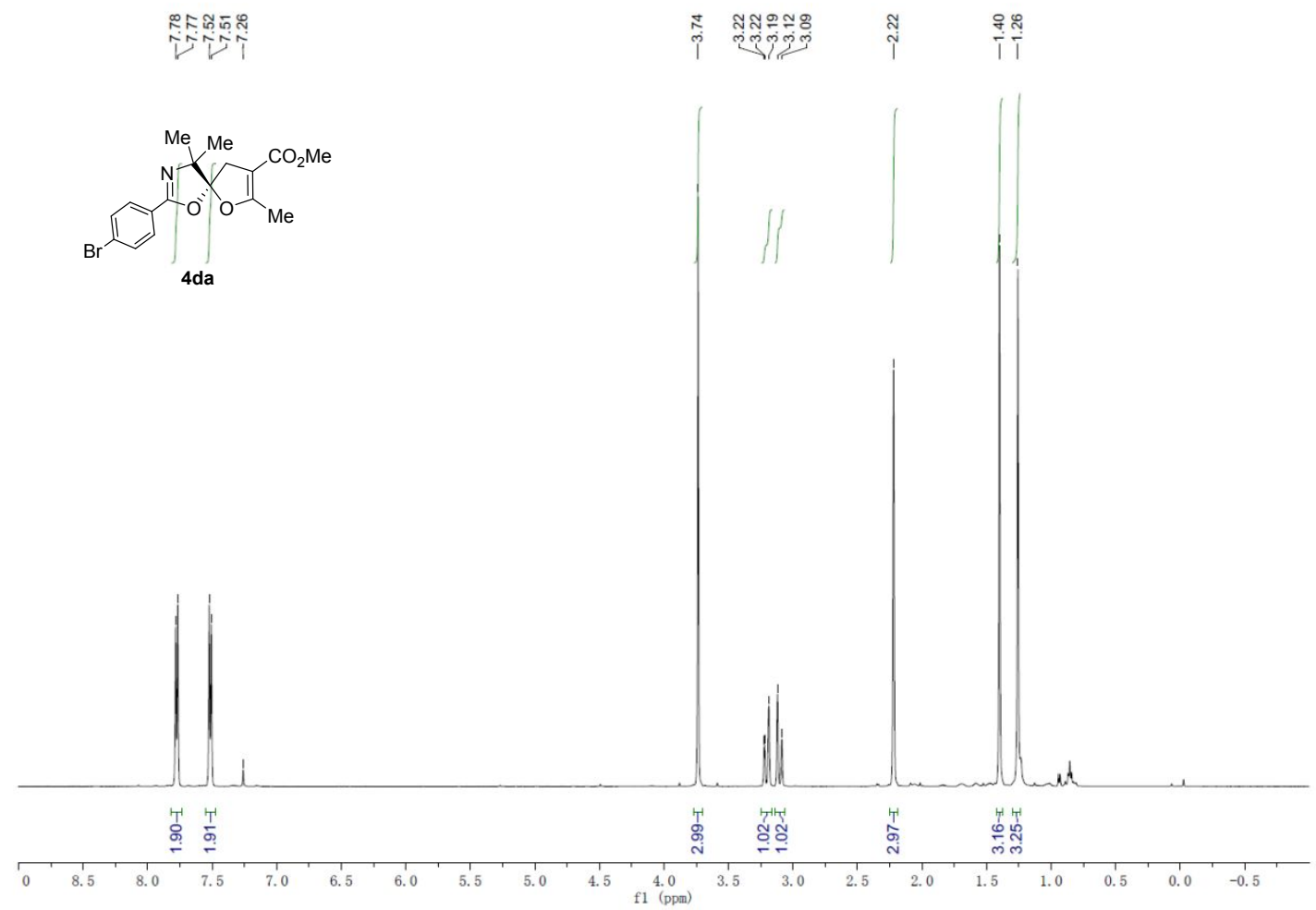

${ }^{13} \mathrm{C}-\mathrm{NMR}\left(125 \mathrm{MHz}, \mathrm{CDCl}_{3}\right)$ of compound 4da

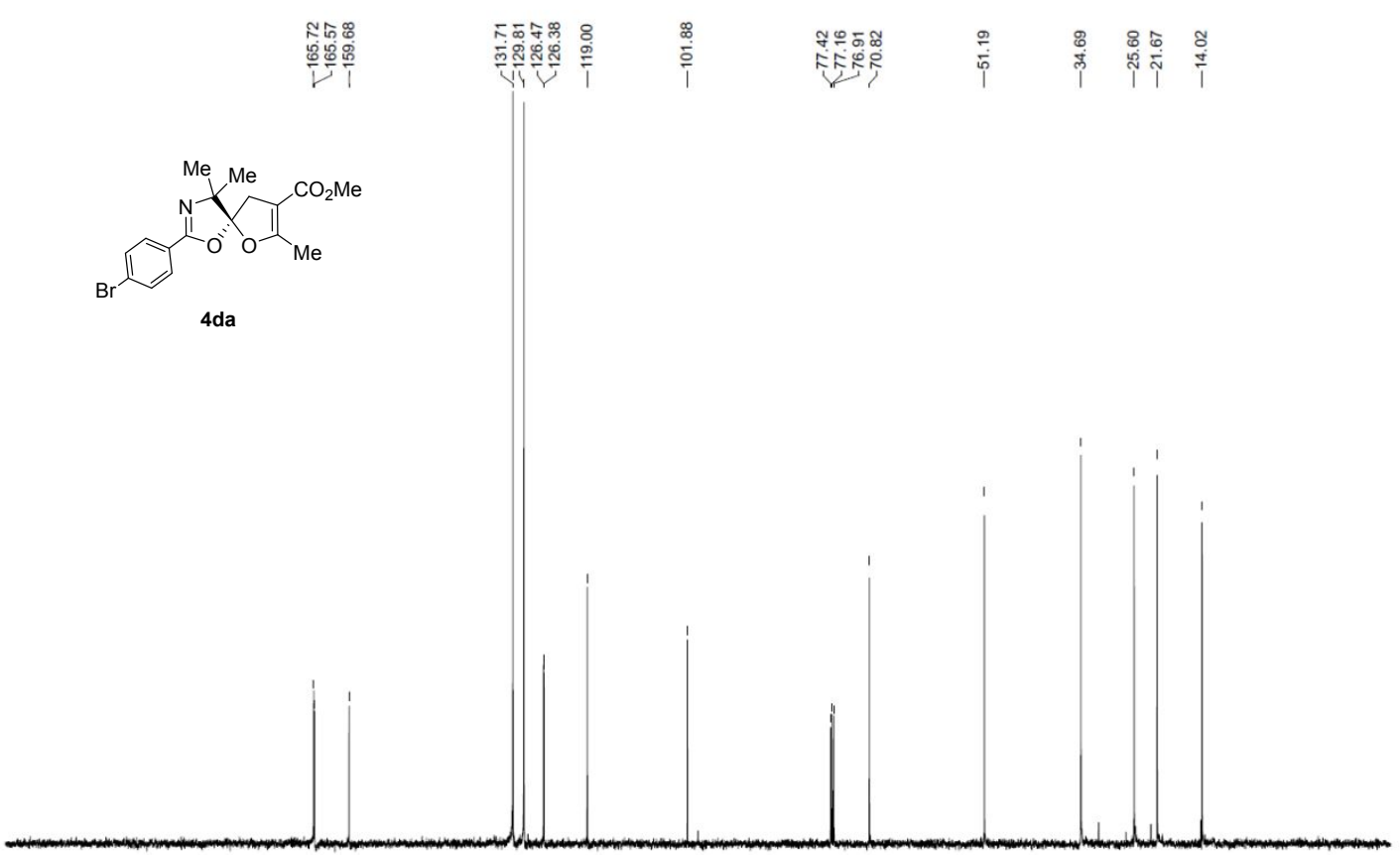

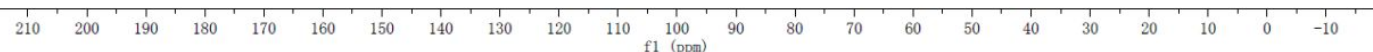


${ }^{1} \mathrm{H}-\mathrm{NMR}\left(500 \mathrm{MHz}, \mathrm{CDCl}_{3}\right.$ ) of compound 4ea

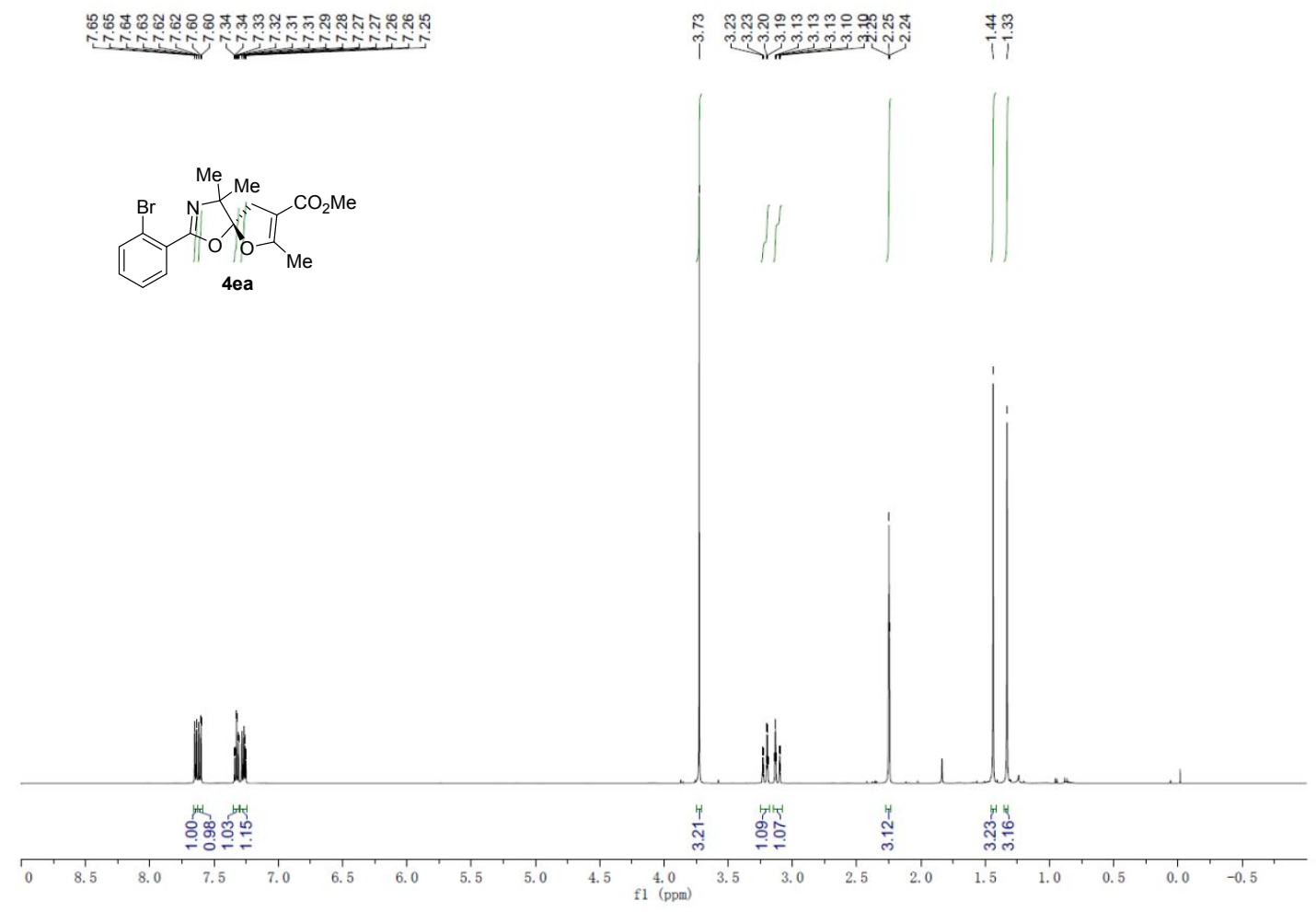

${ }^{13} \mathrm{C}-\mathrm{NMR}$ (125 MHz, $\mathrm{CDCl}_{3}$ ) of compound 4ea

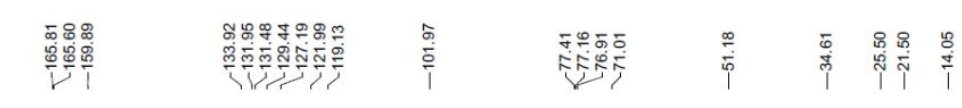
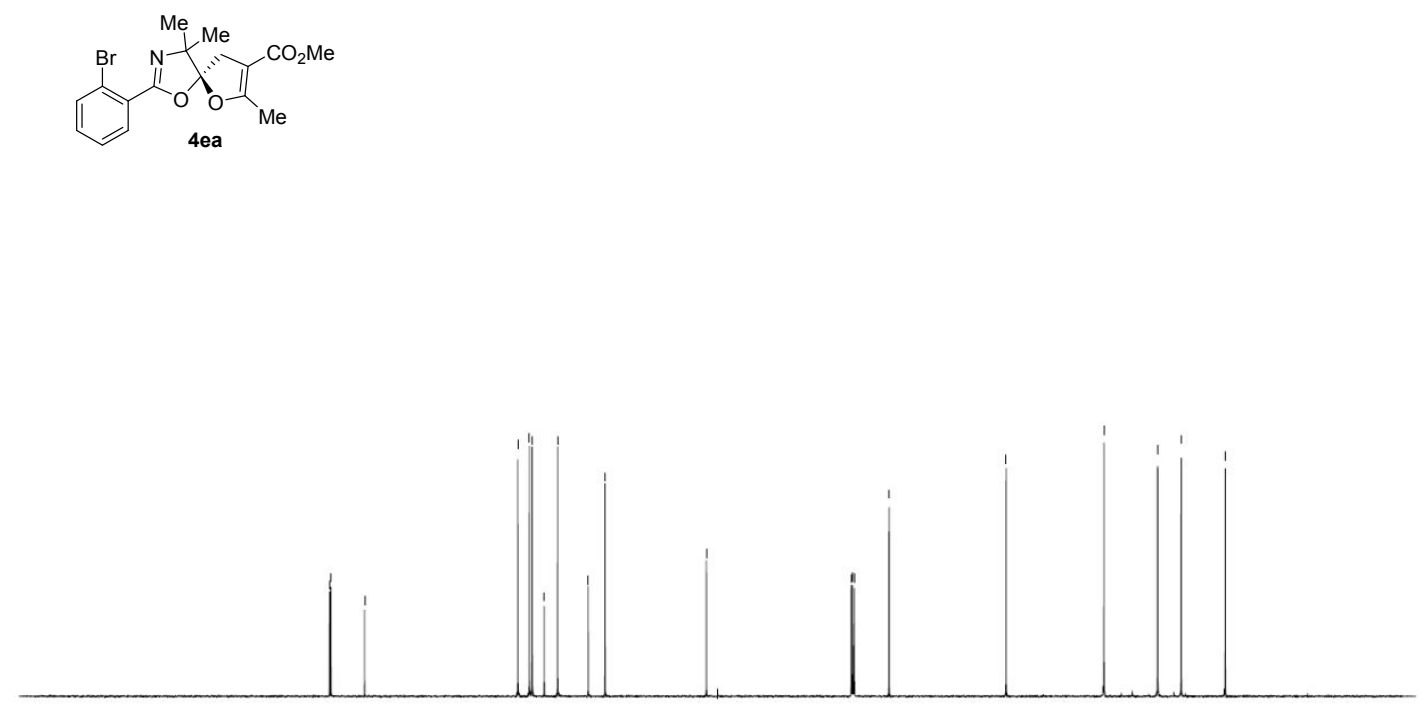

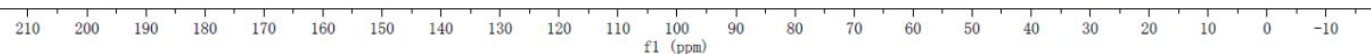


${ }^{1} \mathrm{H}-\mathrm{NMR}\left(500 \mathrm{MHz}, \mathrm{CDCl}_{3}\right)$ of compound $4 \mathrm{fa}$
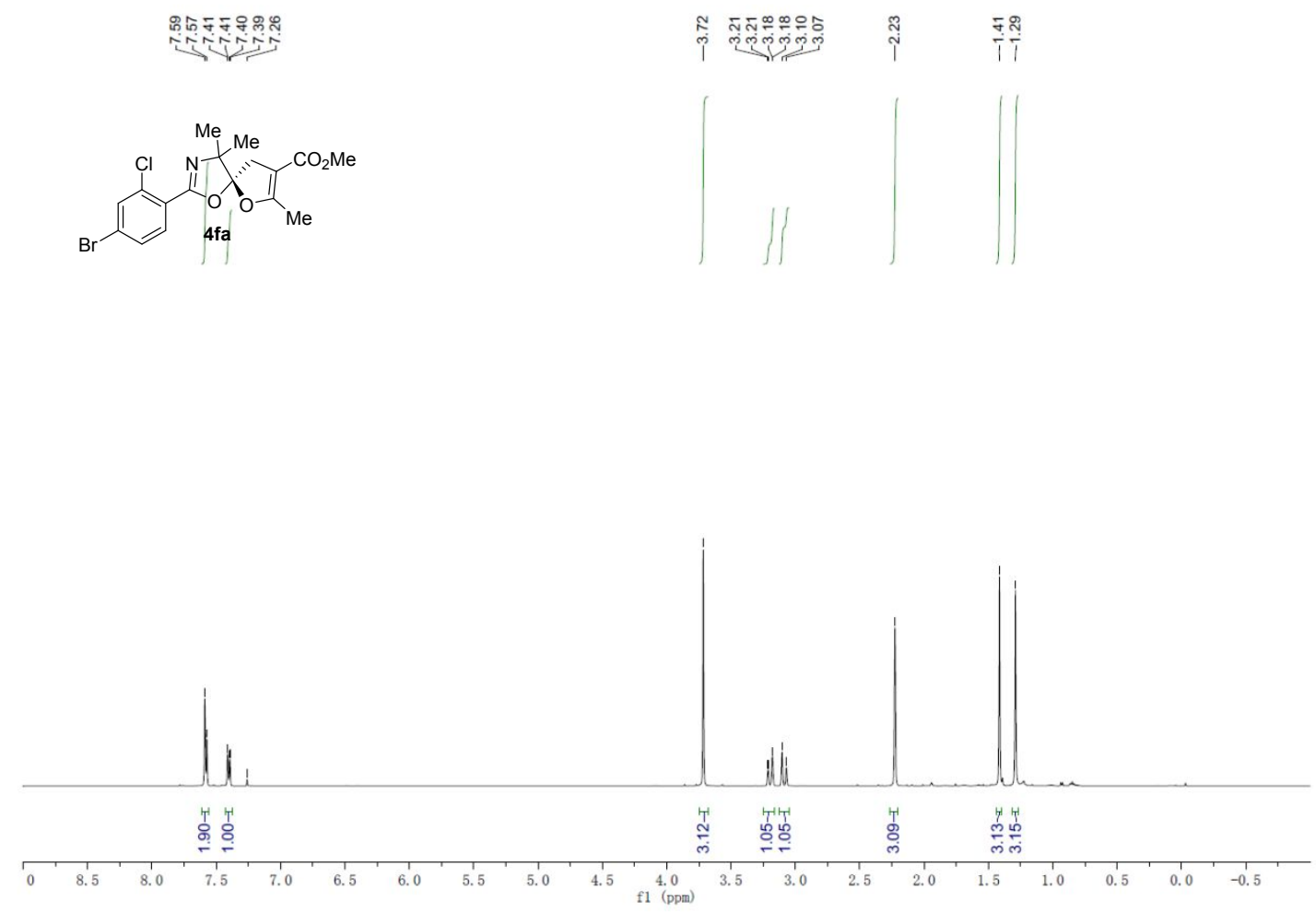

${ }^{13} \mathrm{C}$-NMR (125 $\left.\mathrm{MHz}, \mathrm{CDCl}_{3}\right)$ of compound $4 \mathrm{fa}$

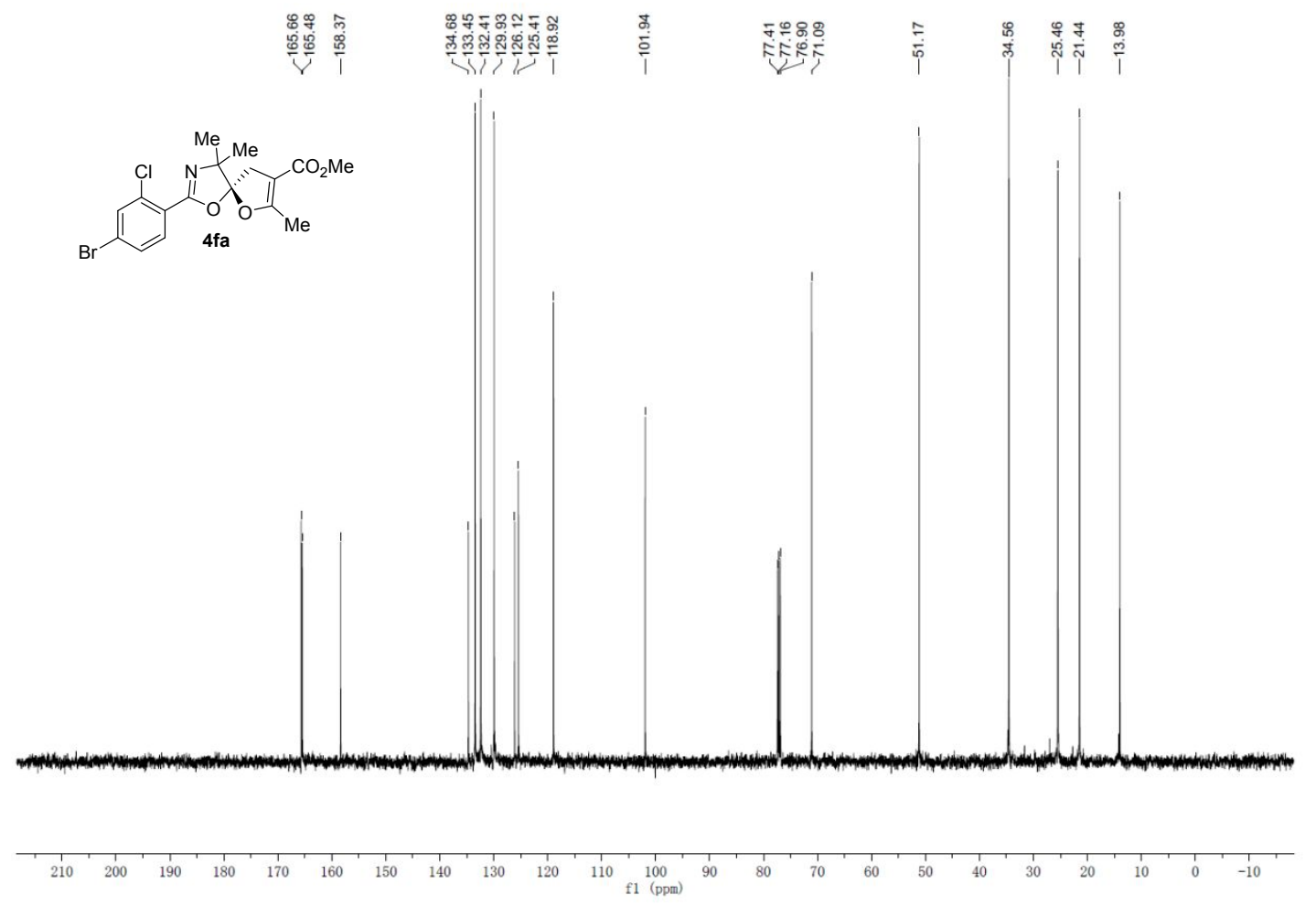


${ }^{1} \mathrm{H}-\mathrm{NMR}\left(500 \mathrm{MHz}, \mathrm{CDCl}_{3}\right)$ of compound 4ga

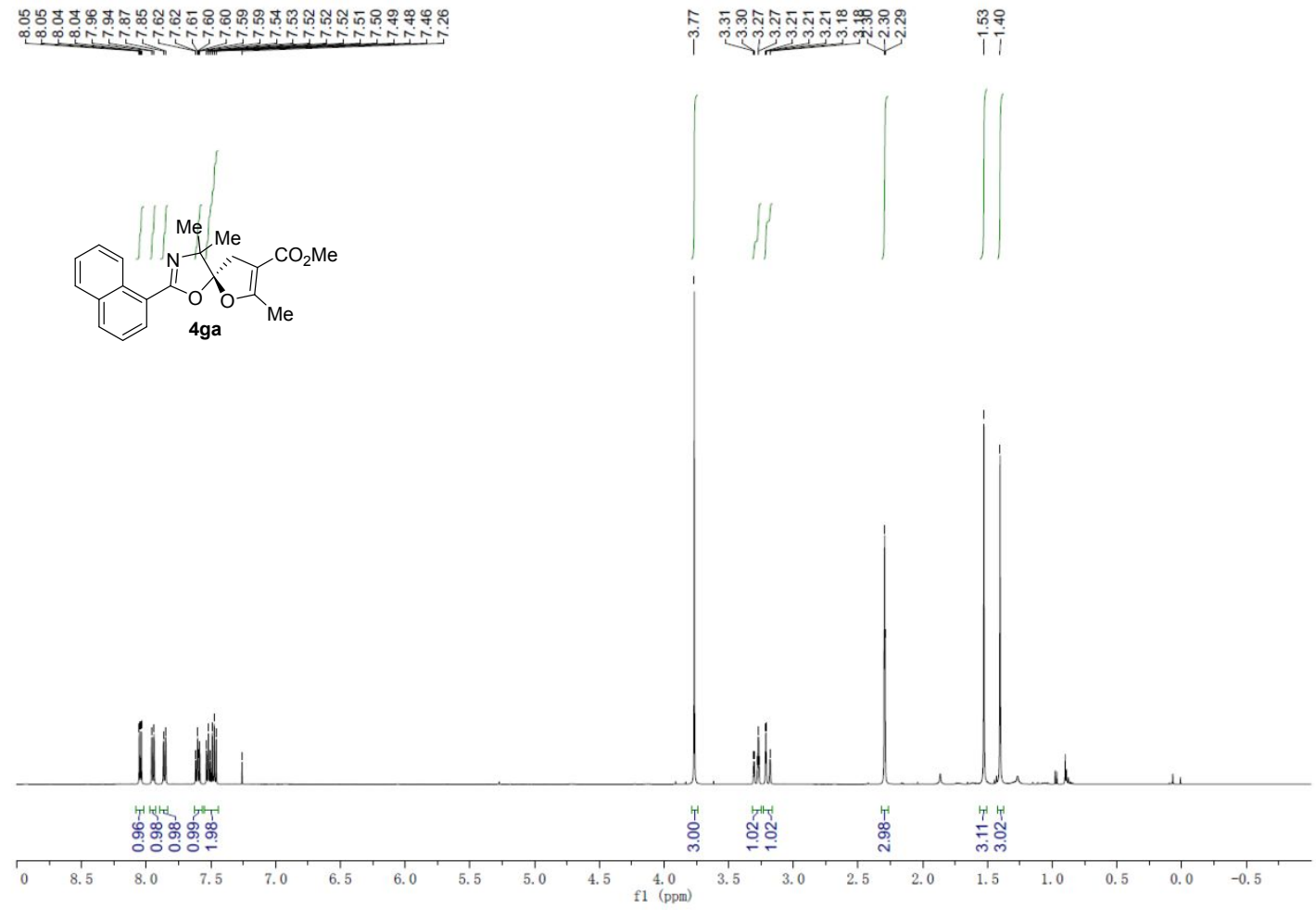

${ }^{13} \mathrm{C}-\mathrm{NMR}\left(125 \mathrm{MHz}, \mathrm{CDCl}_{3}\right)$ of compound 4ga

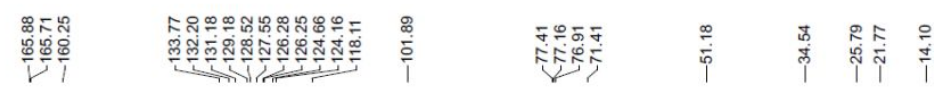
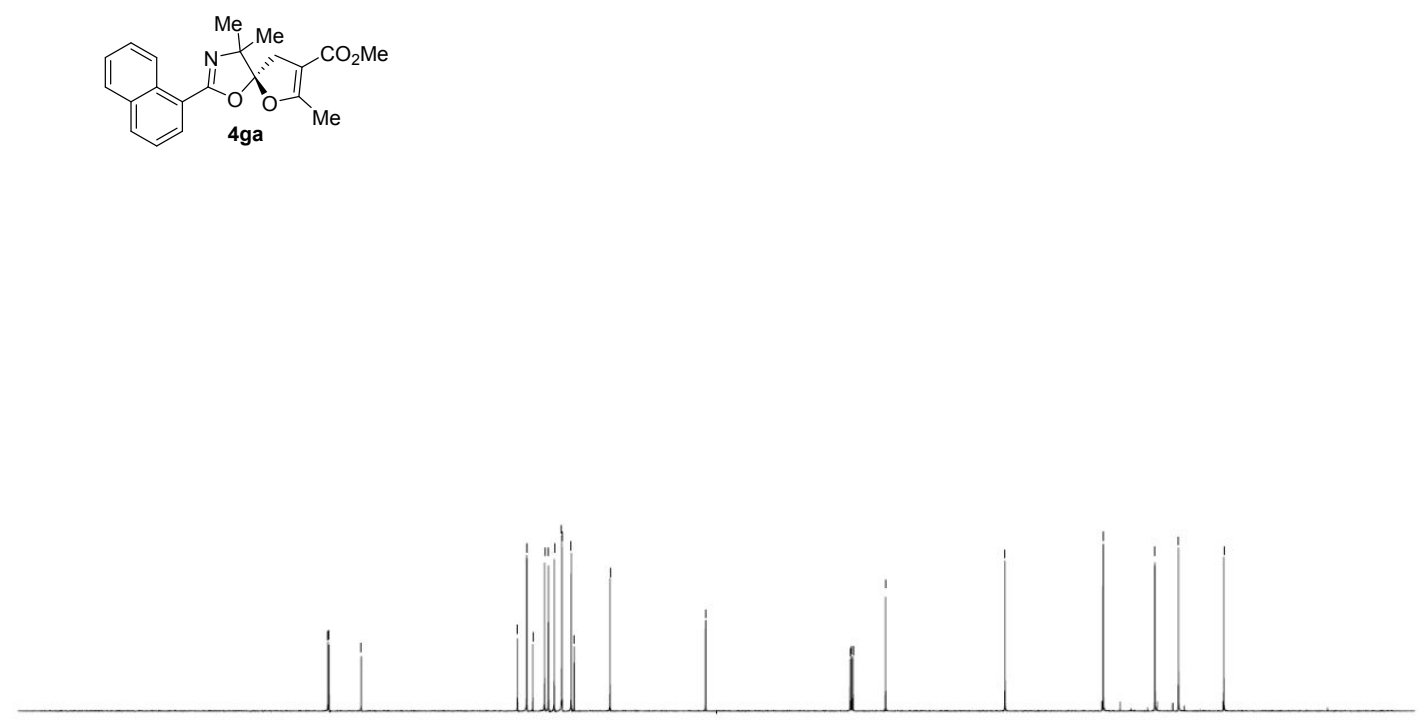

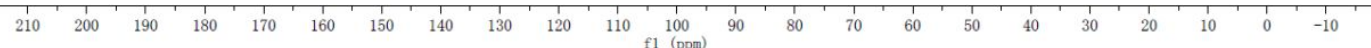


${ }^{1} \mathrm{H}-\mathrm{NMR}\left(500 \mathrm{MHz}, \mathrm{CDCl}_{3}\right)$ of compound $4 \mathrm{ha}$

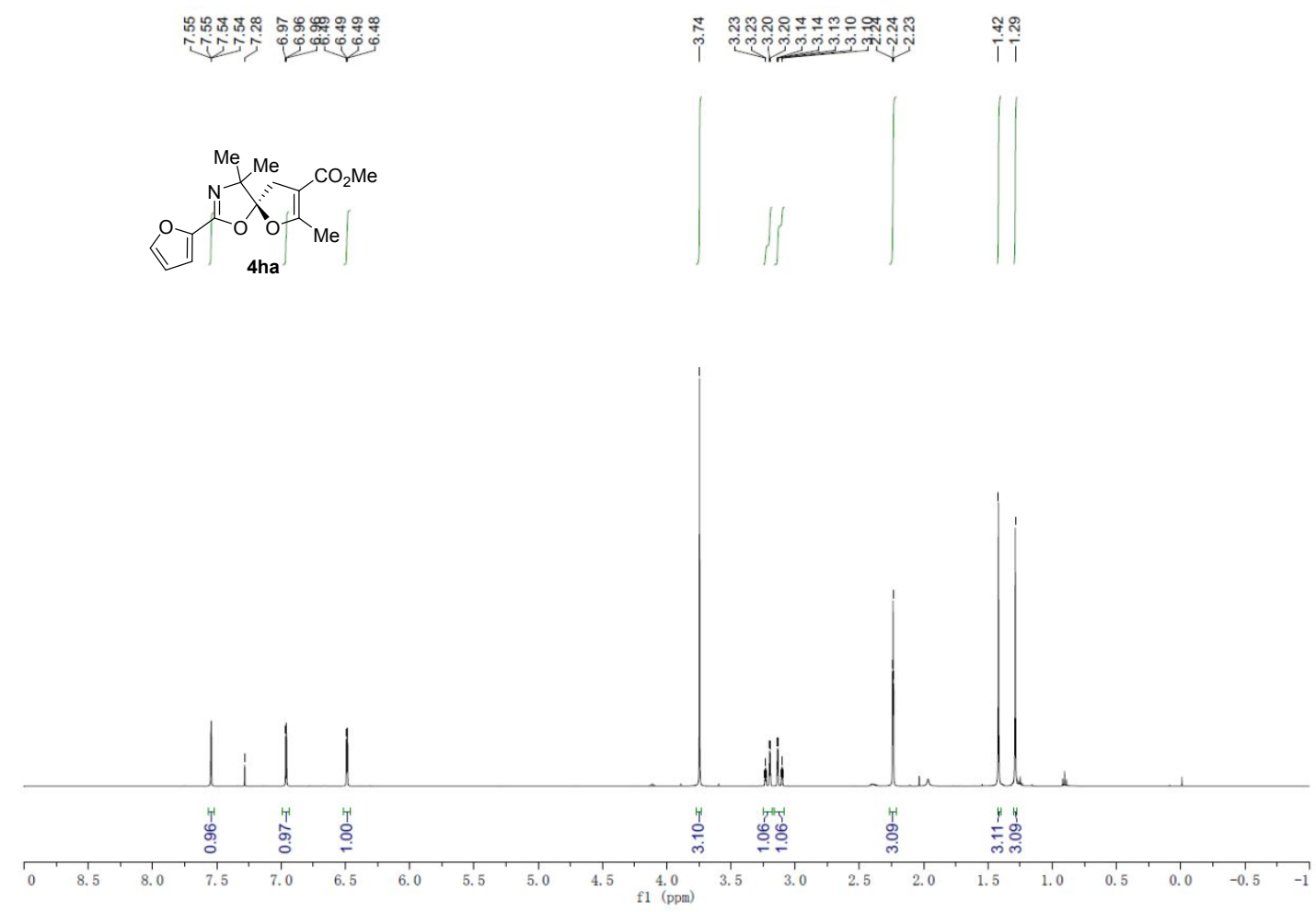

${ }^{13} \mathrm{C}-\mathrm{NMR}\left(125 \mathrm{MHz}, \mathrm{CDCl}_{3}\right)$ of compound $4 \mathrm{ha}$

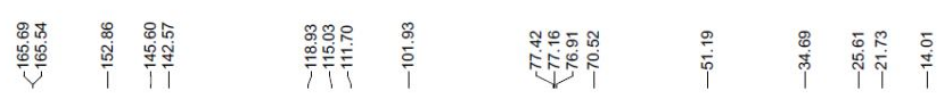
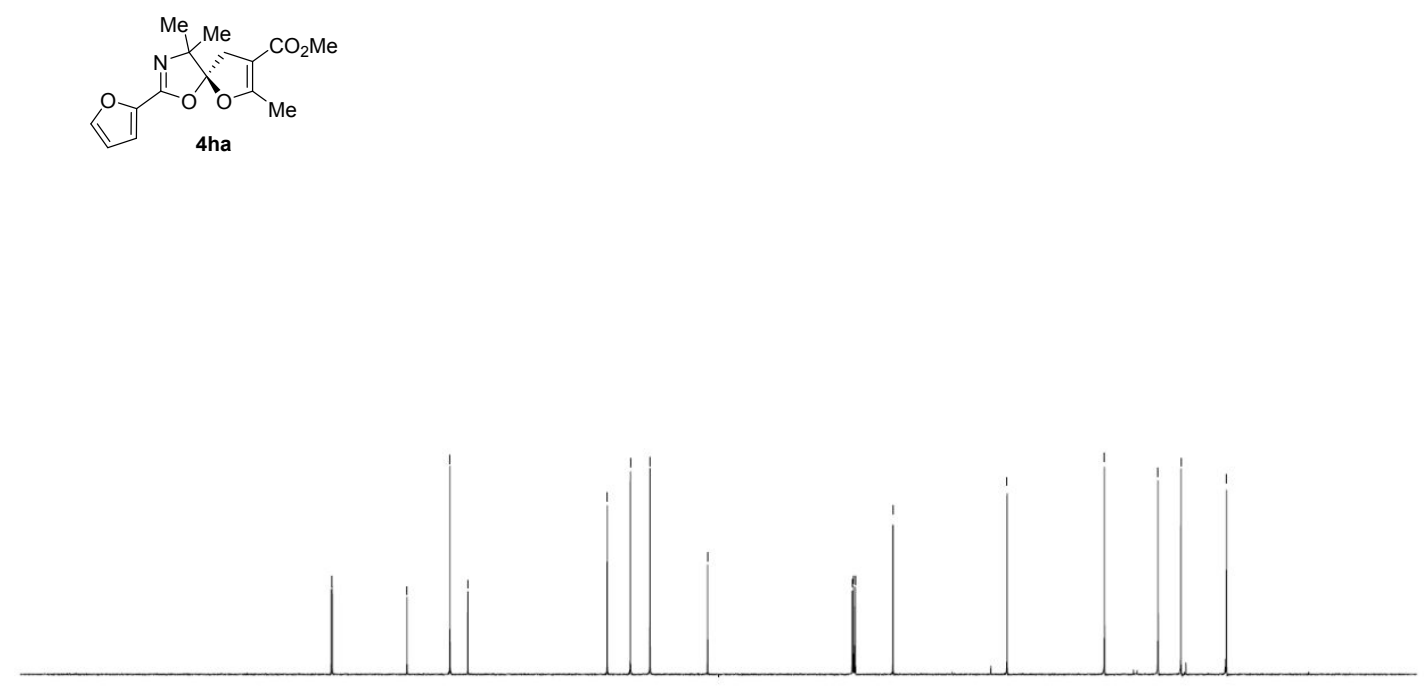

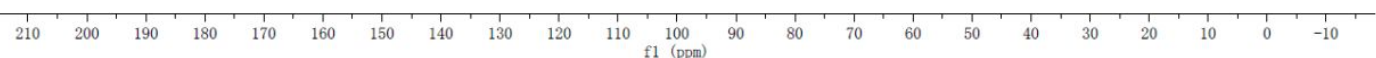


${ }^{1} \mathrm{H}-\mathrm{NMR}\left(500 \mathrm{MHz}, \mathrm{CDCl}_{3}\right)$ of compound 4ia

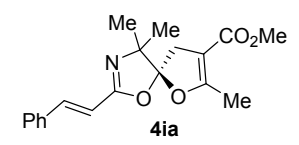

${ }^{13} \mathrm{C}-\mathrm{NMR}\left(125 \mathrm{MHz}, \mathrm{CDCl}_{3}\right)$ of compound 4ia

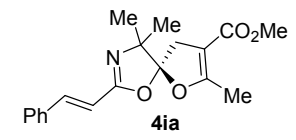


${ }^{1} \mathrm{H}-\mathrm{NMR}\left(500 \mathrm{MHz}, \mathrm{CDCl}_{3}\right)$ of compound $4 \mathrm{ja}$

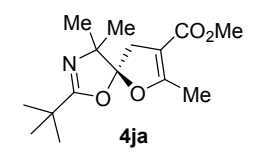

${ }^{13} \mathrm{C}-\mathrm{NMR}\left(125 \mathrm{MHz}, \mathrm{CDCl}_{3}\right)$ of compound 4ja

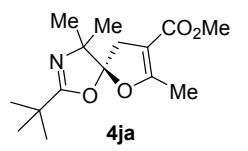


${ }^{1} \mathrm{H}-\mathrm{NMR}\left(500 \mathrm{MHz}, \mathrm{CDCl}_{3}\right)$ of compound 4la

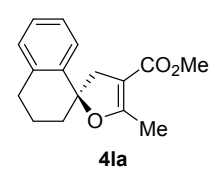

${ }^{13} \mathrm{C}-\mathrm{NMR}\left(125 \mathrm{MHz}, \mathrm{CDCl}_{3}\right)$ of compound 4la

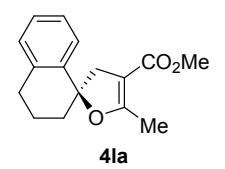


${ }^{1} \mathrm{H}-\mathrm{NMR}\left(500 \mathrm{MHz}, \mathrm{CDCl}_{3}\right.$ ) of compound 6ab

$$
\text { Ph }
$$

${ }^{13} \mathrm{C}-\mathrm{NMR}\left(125 \mathrm{MHz}, \mathrm{CDCl}_{3}\right)$ of compound 6ab

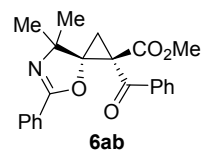


${ }^{1} \mathrm{H}-\mathrm{NMR}\left(500 \mathrm{MHz}, \mathrm{CDCl}_{3}\right.$ ) of compound $6 \mathrm{ad}$

$$
\underbrace{\mathrm{O}}_{\text {Gad }}
$$

${ }^{13} \mathrm{C}-\mathrm{NMR}\left(125 \mathrm{MHz}, \mathrm{CDCl}_{3}\right)$ of compound 6ad

$$
\text { (- }
$$


${ }^{1} \mathrm{H}-\mathrm{NMR}\left(500 \mathrm{MHz}, \mathrm{CDCl}_{3}\right)$ of compound $7 \mathrm{ab}$

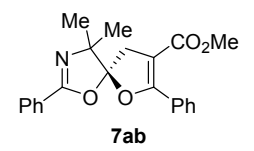

${ }^{13} \mathrm{C}-\mathrm{NMR}\left(125 \mathrm{MHz}, \mathrm{CDCl}_{3}\right)$ of compound 7ab

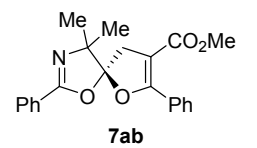


${ }^{1} \mathrm{H}-\mathrm{NMR}\left(500 \mathrm{MHz}, \mathrm{CDCl}_{3}\right)$ of compound $7 \mathrm{ac}$

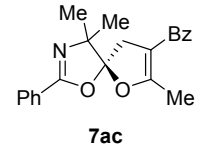

${ }^{13} \mathrm{C}-\mathrm{NMR}\left(125 \mathrm{MHz}, \mathrm{CDCl}_{3}\right.$ ) of compound $7 \mathrm{ac}$

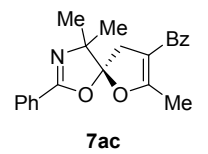


${ }^{1} \mathrm{H}-\mathrm{NMR}\left(500 \mathrm{MHz}, \mathrm{CDCl}_{3}\right)$ of compound 8ka

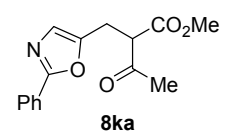

${ }^{13} \mathrm{C}-\mathrm{NMR}\left(125 \mathrm{MHz}, \mathrm{CDCl}_{3}\right)$ of compound 8ka

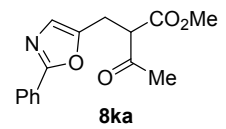


6. HPLC Traces for Racemic and Chiral Compounds
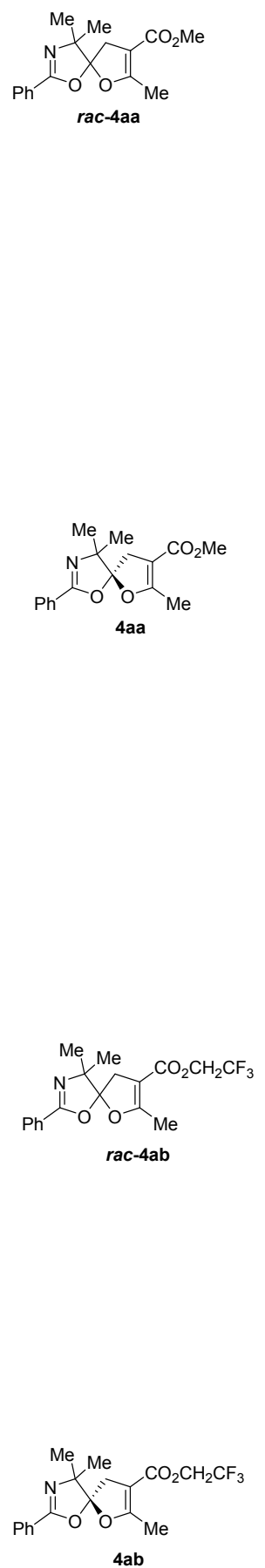


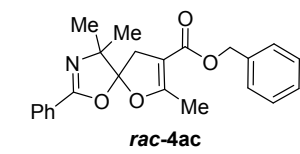

$\overbrace{4 a c}^{M e}$
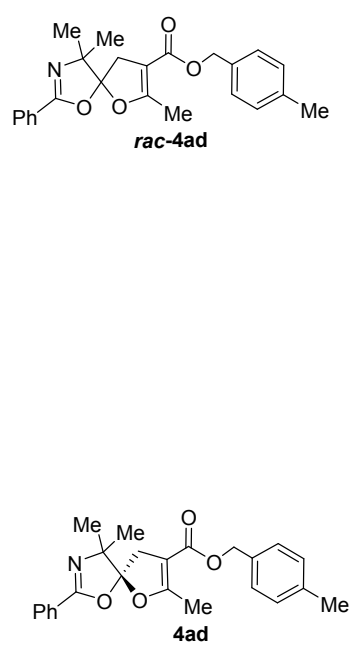
rac-4ae



rac-4af

然 


$$
\text { rac-4ag }
$$
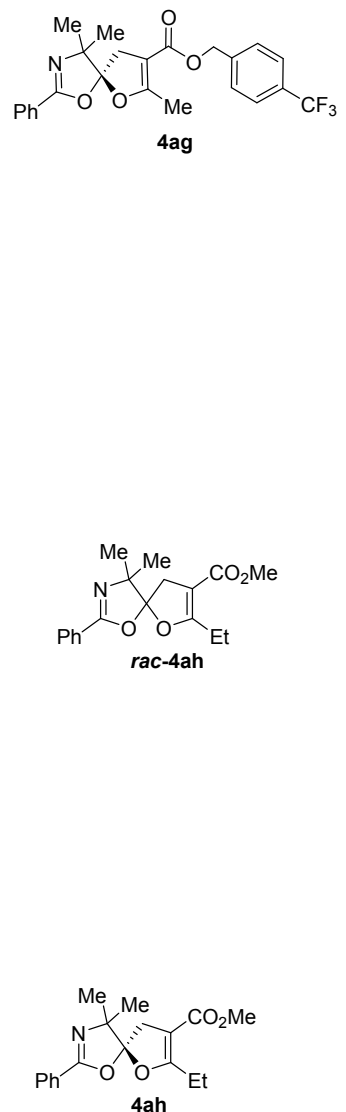


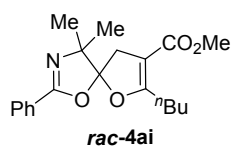

$\overbrace{4 \mathbf{3}}^{\mathrm{Mei}}$
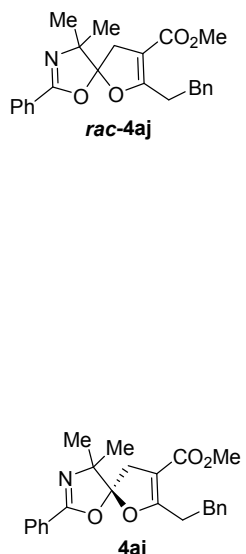


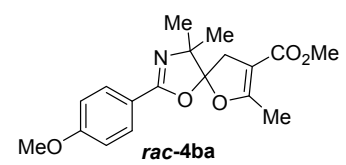

bba
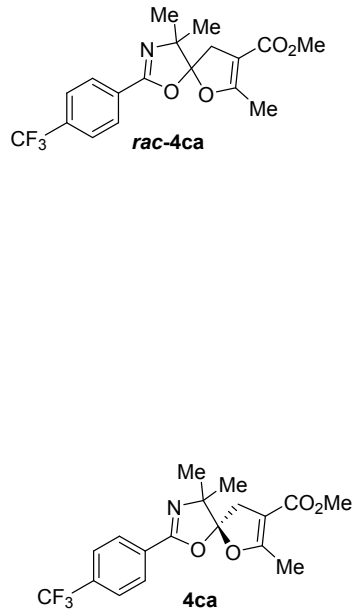

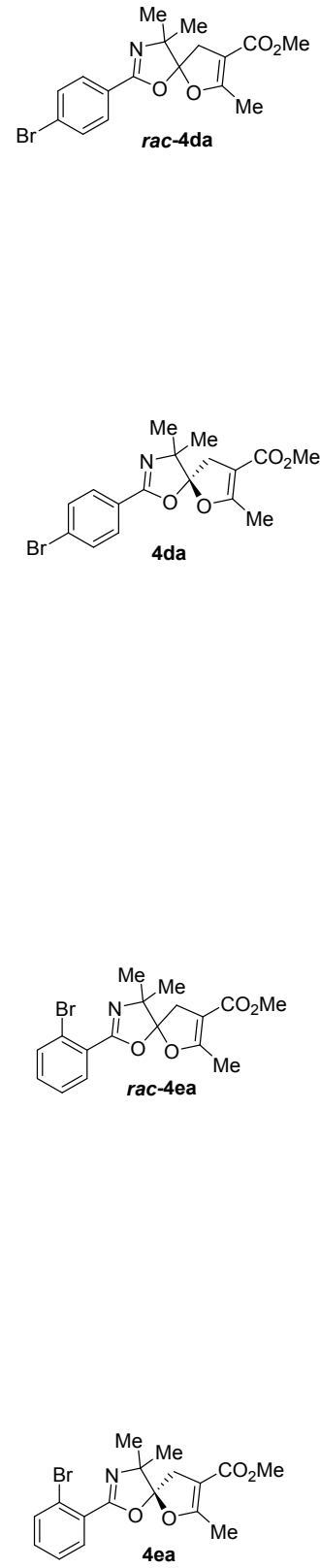

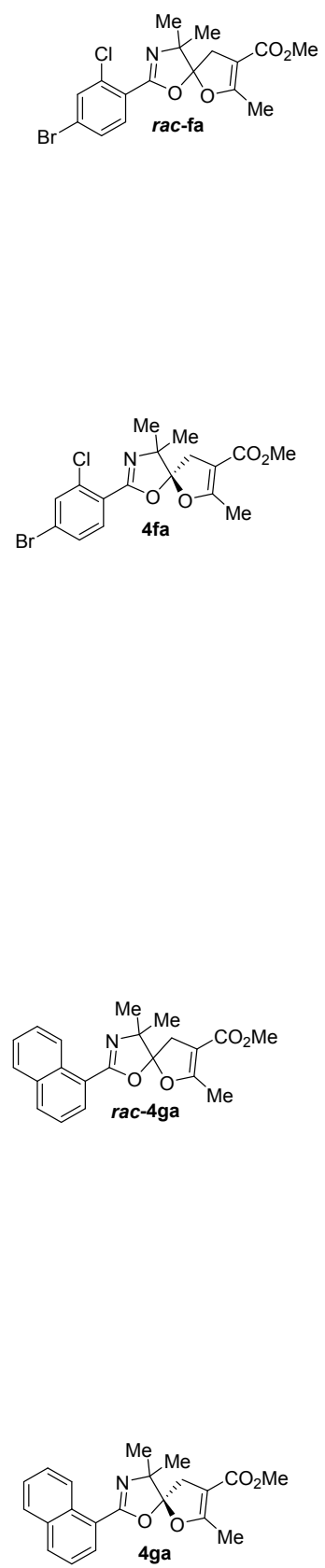

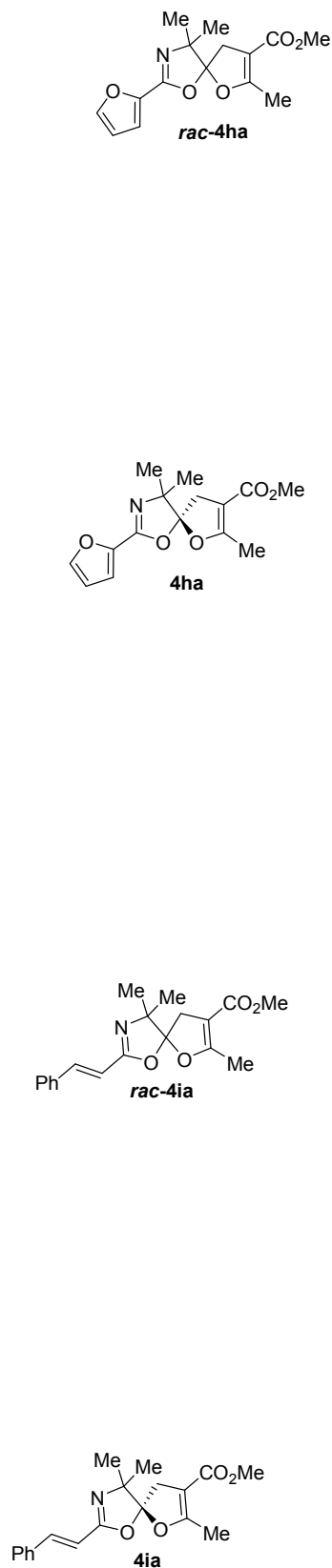

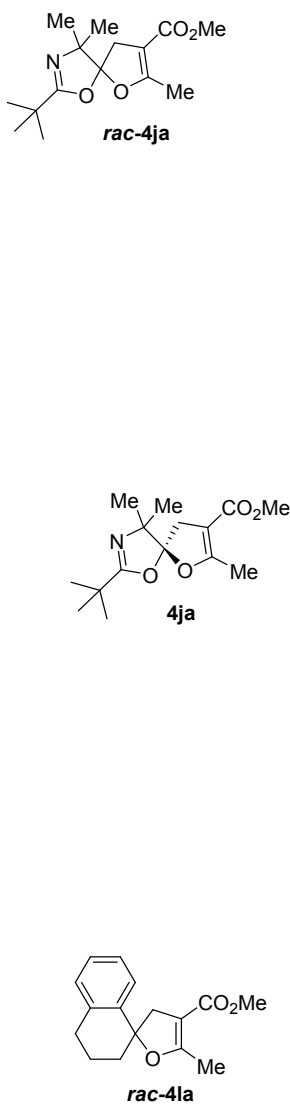

$\sum_{\mathrm{O}}^{\mathrm{I} a} \mathrm{Me}_{\mathrm{Me}}^{\mathrm{CO}_{2} \mathrm{Me}}$ 
rac-6ab

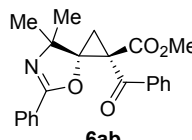

$6 a b$

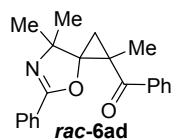

(I) 


\section{X-ray Refinement Data}

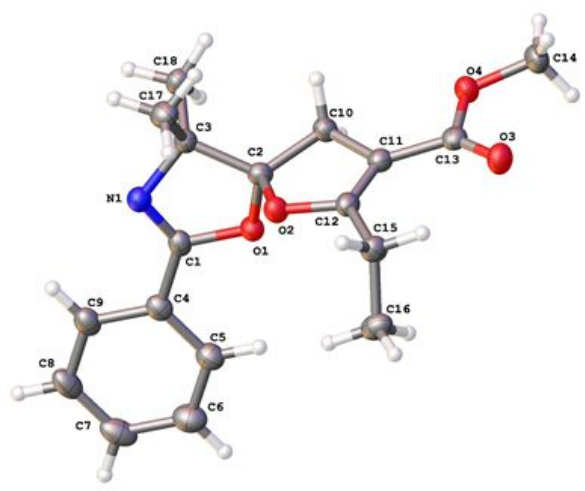<smiles>C#CC1=C(C(C)=O)O[C@@]2(C1)OC(c1ccccc1)=NC2(C)C</smiles>

(S)-4ah

CCDC: 2068308

Figure S1. ORTEP drawing of $(\boldsymbol{S})-4 a h$ showing thermal ellipsoids at the $50 \%$ probability level.

Single crystals of $\mathrm{C}_{18} \mathrm{H}_{21} \mathrm{NO}_{4}$ were prepared by slow evaporation of hexane/DCM solution. A suitable colorless block-like crystal, with dimensions of $0.182 \mathrm{~mm} \times 0.129$ $\mathrm{mm} \times 0.066 \mathrm{~mm}$, was mounted in paratone oil onto a nylon loop. All data were collected at 100.0(1) K, using a XtaLAB Synergy/ Dualflex, HyPix fitted with $\mathrm{CuK} \alpha$ radiation $(\lambda=1.54184 \AA)$. Data collection and unit cell refinement were performed using CrysAlisPro software. ${ }^{[3]}$ The total number of data were measured in the $9.4^{\circ}<2 \theta<$ $152.9^{\circ}$ using $\omega$ scans. Data processing and absorption correction, giving minimum and maximum transmission factors $(0.433,1.000)$ were accomplished with CrysAlisPro ${ }^{[3]}$ and SCALE3 ABSPACK ${ }^{[4]}$, respectively. The structure, using Olex $2^{[5]}$, was solved with the ShelXT ${ }^{[6]}$ structure solution program using direct methods and refined (on $F^{2}$ ) with the ShelXL ${ }^{[7]}$ refinement package using full-matrix, least-squares techniques. All nonhydrogen atoms were refined with anisotropic displacement parameters. All hydrogen atom positions were determined by geometry and refined by a riding model.

Table S2: Crystallographic data and structure refinement for $(\boldsymbol{S})-\mathbf{4 a h}$

\begin{tabular}{|c|c|}
\hline $\begin{array}{c}\text { Identification } \\
\text { code }\end{array}$ & $\mathrm{Hpd} 302(1)$ \\
\hline Empirical formula & $\mathrm{C}_{18} \mathrm{H}_{21} \mathrm{NO}_{4}$ \\
\hline Formula weight & 315.56 \\
\hline Crystal system & Monoclinic \\
\hline Space group & $P 2_{1}$ \\
\hline$a(\AA)$ & $9.52540(10)$ \\
\hline$b(\AA)$ & $9.18680(10)$ \\
\hline$c(\AA)$ & $10.32990(10)$ \\
\hline$\alpha\left(^{\circ}\right)$ & 90 \\
\hline$\beta\left(^{\circ}\right)$ & $114.7850(10)$ \\
\hline$\gamma\left(^{\circ}\right)$ & 90 \\
\hline Volume $\left(\AA^{\circ}\right)$ & $820.683(16)$ \\
\hline
\end{tabular}




\begin{tabular}{|c|c|}
\hline Z & 2 \\
\hline$\rho$ (calc.) & 1.276 \\
\hline$\lambda$ & 1.54184 \\
\hline Temp. (K) & $100.0(1)$ \\
\hline $\mathrm{F}(000)$ & 336 \\
\hline$\mu\left(\mathrm{mm}^{-1}\right)$ & 0.736 \\
\hline $\mathrm{T}_{\min }, \mathrm{T}_{\max }$ & $0.433,1.000$ \\
\hline $2 \theta_{\text {range }}\left({ }^{\circ}\right)$ & 9.4 to 152.9 \\
\hline $\begin{array}{l}\text { Reflections } \\
\text { collected }\end{array}$ & 18194 \\
\hline $\begin{array}{l}\text { Independent } \\
\text { reflections }\end{array}$ & $\begin{array}{c}3193 \\
{[\mathrm{R}(\mathrm{int})=0.0319]}\end{array}$ \\
\hline Completeness & $99.9 \%$ \\
\hline $\begin{array}{c}\text { Data / restraints / } \\
\text { parameters }\end{array}$ & $3193 / 1 / 213$ \\
\hline $\begin{array}{l}\text { Observed data } \\
\qquad[\mathrm{I}>2 \sigma(\mathrm{I})]\end{array}$ & 3136 \\
\hline$w R\left(F^{2}\right.$ all data $)$ & 0.0686 \\
\hline$R(F$ obsd data $)$ & 0.0257 \\
\hline $\begin{array}{l}\text { Goodness-of-fit } \\
\text { on } F^{2}\end{array}$ & 1.01 \\
\hline $\begin{array}{l}\text { largest diff. peak } \\
\text { and hole }\left(\mathrm{e} \AA^{-3}\right)\end{array}$ & $0.19 /-0.12$ \\
\hline
\end{tabular}

\section{Reference}

[1]. (a) Dong, K.; Marichev, K. O.; Xu, X.; Doyle, M. P. Synlett. 2019; 30, 1457-1461. (b) Lu, Q.; Mondal, S.; Cembellín, S.; Glorius, F. Angew. Chem. Int. Ed. 2018, 57, 10732-10736. (c) Dias, R. M.; Oliveira, G. P.; Burtoloso, A. Org. Biomol. Chem., 2020, $18,4815-4823$.

[2]. (a) Senadi, G. C.; Hu, W.-P.; Hsiao, J.-S.; Vandavasi, J. K.; Chen, C. Y.; Wang, J.J. Org. Lett.,2012, 14, 4478. (b) Seppänen, O.; Aikonen, S.; Muuronen, M.; AlamilloFerrer, C.; Burés, J.; Helaja, J. Chem. Commun., 2020, 56, 14697-14700.

[3] CrysAlisPro 1.171.40.63a (Rigaku Oxford Diffraction, 2019)

[4] SCALE3 ABSPACK -An Oxford Diffraction program(1.0.4,gui:1.0.3) (C) 2005 Oxford Diffraction Ltd.

[5] O. V. Dolomanov, L. J. Bourhis, R. J. Gildea, J. A. K. Howard, H. Puschmann, J. Appl. Cryst. 2009, 42, 339.

[6] G. M. Sheldrick, Acta Cryst. 2015, A71, 3. 
[7] G. M. Sheldrick, Acta Cryst. 2008, A64, 112. 